\title{
Types and Amounts of Complementary Foods and Beverages and Food Allergy, Atopic Dermatitis/Eczema, Asthma, and Allergic Rhinitis: A Systematic Review
}

The Pregnancy and Birth to 24 Months Project

Published date: April 15, 2019

Nutrition Evidence Systematic Review Center for Nutrition Policy and Promotion

Food and Nutrition Service

U.S. Department of Agriculture

3101 Park Center Drive

Alexandria, Virginia 
This systematic review was conducted for the Pregnancy and Birth to 24 Months Project (P/B-24 Project) by the Nutrition Evidence Systematic Review (NESR) team at the Center for Nutrition Policy and Promotion, Food and Nutrition Service, USDA. All systematic reviews from the P/B-24 Project are available on the NESR website: https://nesr.usda.gov.

Conclusion statements drawn as part of this systematic review describe the state of science related to the specific question examined. Conclusion statements do not draw implications, and should not be interpreted as dietary guidance.

The contents of this document may be used and reprinted without permission. Endorsements by NESR, the Center for Nutrition Policy and Promotion, the Food and Nutrition Service, or the U.S. Department of Agriculture (USDA) of derivative products developed from this work may not be stated or implied.

In accordance with Federal civil rights law and USDA civil rights regulations and policies, the USDA, its Agencies, offices, and employees, and institutions participating in or administering USDA programs are prohibited from discriminating based on race, color, national origin, religion, sex, gender identity (including gender expression), sexual orientation, disability, age, marital status, family/parental status, income derived from a public assistance program, political beliefs, or reprisal or retaliation for prior civil rights activity, in any program or activity conducted or funded by USDA (not all bases apply to all programs). Remedies and complaint filing deadlines vary by program or incident.

Persons with disabilities who require alternative means of communication for program information (e.g., Braille, large print, audiotape, American Sign Language, etc.) should contact the responsible Agency or USDA's TARGET Center at (202) 720-2600 (voice and TTY) or contact USDA through the Federal Relay Service at (800) 877-8339. Additionally, program information may be made available in languages other than English.

To file a program discrimination complaint, complete the USDA Program Discrimination Complaint Form, AD3027, found online at How to File a Program Discrimination Complaint and at any USDA office or write a letter addressed to USDA and provide in the letter all of the information requested in the form. To request a copy of the complaint form, call (866) 632-9992. Submit your completed form or letter to USDA by: (1) mail: U.S.

Department of Agriculture, Office of the Assistant Secretary for Civil Rights, 1400 Independence Avenue, SW, Washington, D.C. 20250-9410; (2) fax: (202) 690-7442; or (3) email: program.intake@usda.gov.

USDA is an equal opportunity provider, employer, and lender.

Suggested citation for this systematic review: Nutrition Evidence Systematic Review Team and Complementary Feeding Technical Expert Collaborative. Types and Amounts of Complementary Foods and Beverages and Food Allergy, Atopic Dermatitis/Eczema, Asthma, and Allergic Rhinitis: A Systematic Review. Pregnancy and Birth to 24 Months Project. Alexandria, VA: U.S. Department of Agriculture, Food and Nutrition Service, Center for Nutrition Policy and Promotion, February 2019. Available at: https://nesr.usda.gov/projectspecific-overview-pb-24-0

This systematic review has also been published in the American Journal of Clinical Nutrition: Obbagy JE, English LK, Wong YP, Butte NF, Dewey KG, Fleischer DM, et al. Complementary feeding and food allergies, atopic dermatitis and eczema, asthma, and allergic rhinitis: a systematic review. Am J Clin Nutr. 2019;109(7):890S-934S. doi: 10.1093/ajcn/nqy220.

\section{Related citations are published in the American Journal of Clinical Nutrition:}

- P/B-24 Project overview: Stoody EE, Spahn JM, Casavale KO. The Pregnancy and Birth to 24 Months Project: a series of systematic reviews on diet and health. Am J Clin Nutr. 2019;109(7):685S97S. doi: 10.1093/ajcn/nay372.

- P/B-24 systematic review methodology: Obbagy JE, Spahn JM, Wong YP, Psota TL, Spill MK, Dreibelbis C, et al. Systematic review methodology used in the Pregnancy and Birth to 24 Months Project. Am J Clin Nutr. 2019;109(7):698S-704S. doi: 10.1093/ajcn/nqy226.

- Related systematic reviews from the P/B-24 Project:

- English LK, Obbagy JE, Wong YP, Butte NF, Dewey KG, Fox MK, et al. Timing of introduction 
of complementary foods and beverages and growth, size, and body composition: a systematic review. Am J Clin Nutr. 2019;109(7):935S-55S. doi: 10.1093/ajcn/nqy267.

- English LK, Obbagy JE, Wong YP, Butte NF, Dewey KG, Fox MK, et al. Types and amounts of complementary foods and beverages consumed and growth, size, and body composition: a systematic review. Am J Clin Nutr. 2019;109(7):956S-77S. doi: 10.1093/ajcn/nqy281.

- English LK, Obbagy JE, Wong YP, Butte NF, Dewey KG, Fox MK, et al. Complementary feeding and developmental milestones: a systematic review. Am J Clin Nutr. 2019;109(7):879S-89S. doi: 10.1093/ajcn/nqy321.

- Obbagy JE, English LK, Psota TL, Wong YP, Butte NF, Dewey KG, et al. Complementary feeding and micronutrient status: a systematic review. Am J Clin Nutr. 2019;109(7):852S71S. doi: 10.1093/ajcn/nqy266.

- Obbagy JE, English LK, Wong YP, Butte NF, Dewey KG, Fox MK, et al. Complementary feeding and bone health: a systematic review. Am J Clin Nutr. 2019:872S-8S. doi: 10.1093/ajcn/nqy227. 


\section{ACKNOWLEDGEMENTS}

\section{Complementary Feeding Technical Expert Collaborative (TEC):}

- Nancy F. Butte, PhD, RD, United States Department of Agriculture /Agricultural Research Service, Children's Nutrition Research Center, Baylor College of Medicine, Department of Pediatrics, Emeritus

- Kathryn G. Dewey, PhD, University of California, Davis, Department of Nutrition

- David M. Fleischer, MD, Children's Hospital Colorado, University of Colorado School of Medicine, Department of Pediatrics, Section of Allergy and Immunology

- Mary Kay Fox, Med, Mathematica Policy Research

- Frank R. Greer, MD, University of Wisconsin School of Medicine and Public Health, Department of Pediatrics, Emeritus

- Nancy F. Krebs, MD, MS, University of Colorado School of Medicine, Department of Pediatrics

- Kelley S. Scanlon, PhD, RD, United States Department of Agriculture, Food and Nutrition Service (formerly of the Centers for Disease Control and Prevention, Division of Nutrition, Physical Activity, and Obesity)

\section{Nutrition Evidence Systematic Review (NESR) team:}

- Julie E. Obbagy, PhD, RD USDA, Lead Analyst (05/2016-project completion)

- Laural K. Englishi, PhD, Panum Group, Analyst (11/2016-project completion)

- Tricia L. Psota, USDA, Lead Analyst (07/2015-06/2016)

- Perrine Nadaudi, MS, Panum Group, Analyst (07/2015-05/2016)

- Kirsten Johnsi, MS, USDA, Panum Group, Analyst (07/2015-05/2016)

- Yat Ping Wong, MLS, MPH, USDA, Librarian

- Nancy Terry, MLS, NIH, Librarian

\section{Project Lead:}

- Eve Essery Stoody, PhD, USDA

\section{Federal Expert Group (FEG)-Technical Expert Collaborative (TEC) Liaisons:}

- Kelley S. Scanlon, PhD, RD, United States Department of Agriculture, Food and Nutrition Service (formerly of the Centers for Disease Control and Prevention, Division of Nutrition, Physical Activity, and Obesity)

All TEC and NESR team members, Project leads, and FEG-TEC liaisons participated in establishing the research questions, analytic framework, and study inclusion and exclusion criteria. JEO, LKE, TLP, PN, KJ, YWP, and NT developed and conducted the literature search, screened search results, and identified studies for inclusion. JEO and LKE extracted data and assessed risk of bias for included studies. NFC, KGD, MKF, FRG, NFK, DF and KSS reviewed and provided substantive feedback on all systematic review materials, including the synthesis of the body of evidence, conclusion statement,

i Under contract with the Food and Nutrition Service, United States Department of Agriculture. 
and grade of the strength of the evidence. JEO prepared this report and EES provided oversight. All authors critically reviewed and approved the final report. The authors declare no conflicts of interest.

FUNDING SOURCE: United States Department of Agriculture, Food and Nutrition Service, Center for Nutrition Policy and Promotion, Alexandria, VA 


\section{TABLE OF CONTENTS}

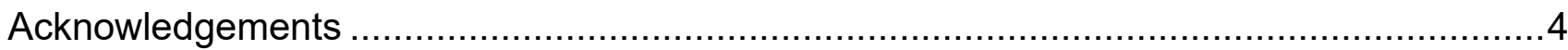

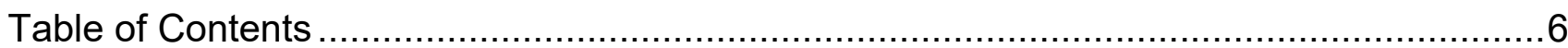

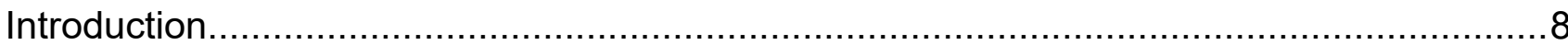

What is the relationship between types and amounts of introduction of complementary foods and beverages (CFB) and food allergy, atopic dermatitis/eczema, asthma, and

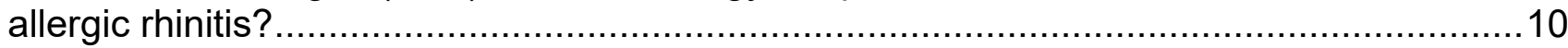

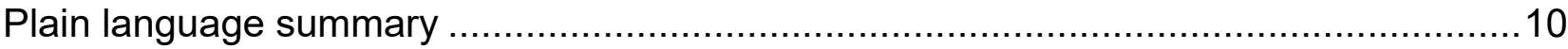

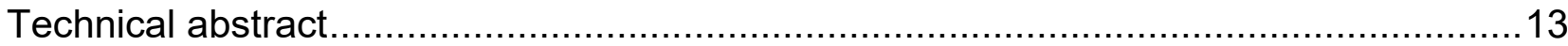

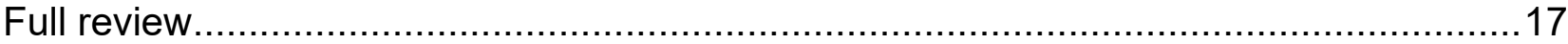

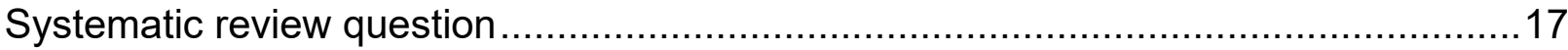

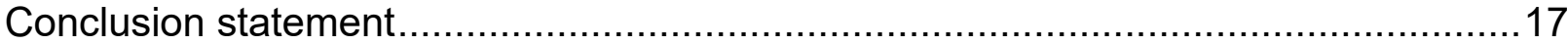

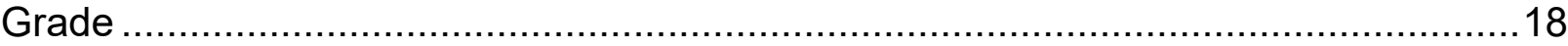

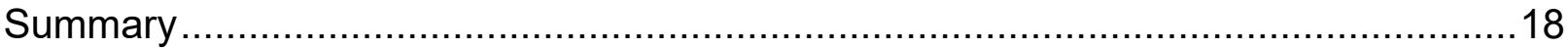

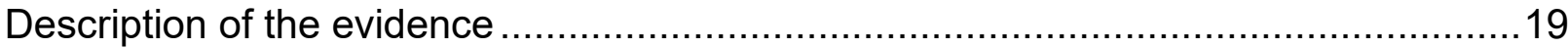

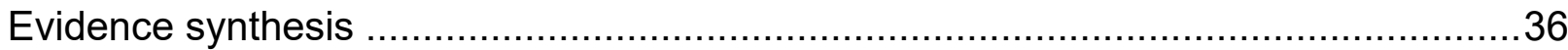

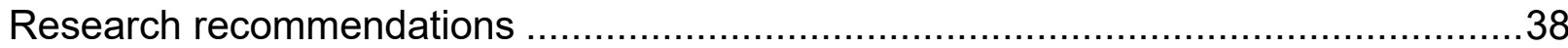

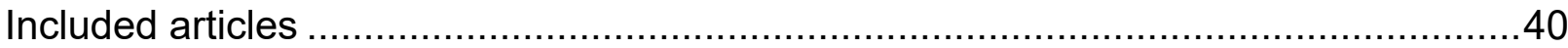

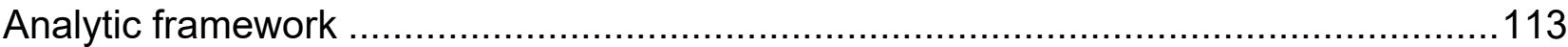

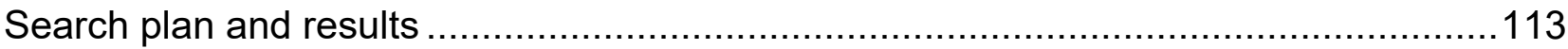

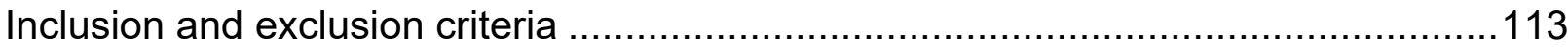

Search terms and electronic databases used ..................................................115

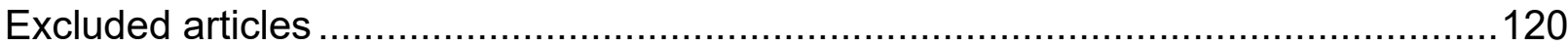

Table 1. Description of studies that examined the relationship between consumption of specific types of complementary foods and beverages (CFB) and risk of atopic disease ...43 Table 2. Summary of studies examining the relationship between diet diversity or dietary patterns during the complementary feeding period and food allergy, atopic dermatitis/eczema, asthma, and allergic rhinitis ....

Table 3. Results of studies that examined the relationship between peanut, tree nut, and/or seed product consumption during the complementary feeding period and risk of atopic

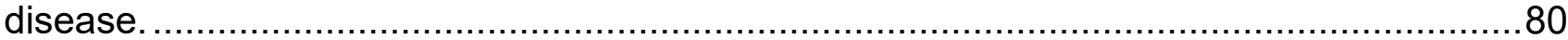
Table 4. Results of studies that examined the relationship between egg consumption during the complementary feeding period and risk of atopic disease.

Table 5. Results of studies that examined the relationship between fish and/or shellfish consumption during the complementary feeding period and risk of atopic disease.

Table 6. Results of studies that examined the relationship between milk product consumption during the complementary feeding period and risk of atopic disease

Table 7. Results of studies that examined the relationship between wheat consumption 
during the complementary feeding period and risk of atopic disease.

97

Table 8. Results of studies that examined the relationship between soybean consumption

during the complementary feeding period and risk of atopic disease.

Table 9. Results of studies that examined the relationship between consumption of complementary foods and beverages not considered to be major allergens and risk of atopic disease.

102

Table 10. Results of studies examining the relationship between diet diversity and dietary patterns during the complementary feeding period and food allergy, atopic dermatitis, asthma, and allergic rhinitis. 109

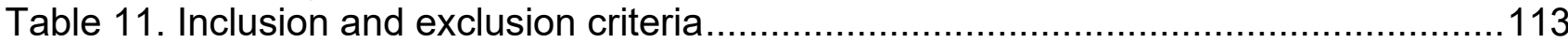

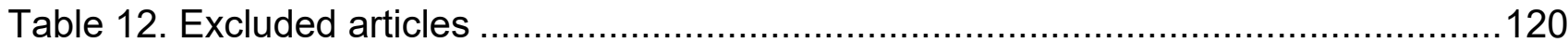

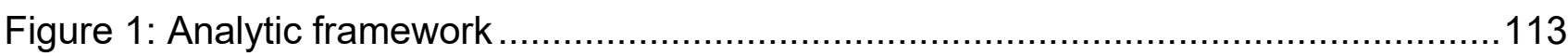

Figure 2: Flow chart of literature search and screening results .................................119 


\section{INTRODUCTION}

This document describes a systematic review conducted to answer the following question: What is the relationship between types and amounts of complementary foods and beverages (CFB) and food allergy, atopic dermatitis/eczema, asthma, and allergic rhinitis? This systematic review was conducted as part of the Pregnancy and Birth to 24 Months (P/B-24) Project by USDA's Nutrition Evidence Systematic Review (NESR).

The purpose of the P/B-24 Project was to conduct a series of systematic reviews on diet and health for women who are pregnant and for infants and toddlers from birth to 24 months of age. This project was a joint initiative led by USDA and HHS, and USDA's NESR carried out all of the systematic reviews. A Federal Expert Group (FEG), a broadly representative group of Federal researchers and program leaders, also provided input throughout the P/B-24 Project. More information about the P/B-24 Project has been publishedi and is available on the NESR website: https://nesr.usda.gov/project-specificoverview-pb-24-0.

NESR, formerly known as the Nutrition Evidence Library (NEL), specializes in conducting food- and nutrition-related systematic reviews using a rigorous, protocol-driven methodology. To conduct each P/B-24 systematic review, NESR's staff worked with a Technical Expert Collaborative (TEC), which is a group of 7-8 leading subject matter experts.

NESR's systematic review methodology involves developing and prioritizing systematic review questions, searching for and selecting studies, extracting and assessing the risk of bias of data from each included study, synthesizing the evidence, developing a conclusion statement, grading the evidence underlying the conclusion statement, and recommending future research. A detailed description of the methodology used in conducting systematic reviews for the P/B-24 Project has been publishediii and is available on the NESR website: https://nesr.usda.gov/pb-24-project-methodology-0. In addition, starting on page 112, this document includes details about the methodology as it was applied to the systematic review described herein. An analytic framework that illustrates the overall scope of the question, including the population, the interventions and/or exposures, comparators, and outcomes of interest, is found on page 112. In addition, the literature search plan that was used to identify studies included in this systematic review is found on page 112 .

\footnotetext{
ii Stoody EE, Spahn JM, Casavale KO. The Pregnancy and Birth to 24 Months Project: a series of systematic reviews on diet and health. Am J Clin Nutr. 2019;109(7):685S-97S. doi: 10.1093/ajcn/nqy372.

iii Obbagy JE, Spahn JM, Wong YP, Psota TL, Spill MK, Dreibelbis C, et al. Systematic review methodology used in the Pregnancy and Birth to 24 Months Project. Am J Clin Nutr. 2019;109(7):698S-704S. doi: 10.1093/ajcn/nqy226.
} 


\section{List of abbreviations}

\begin{tabular}{ll}
\hline Abbreviation & Full name \\
\hline BF & Breastfed \\
\hline CF & Complementary feeding \\
\hline CFB & Complementary foods and beverages \\
\hline FEG & Federal Expert Group \\
\hline FF & Formula fed \\
\hline HHS & Department of Health and Human Services \\
\hline IgE & Immunoglobulin E \\
\hline NEL & Nutrition Evidence Library \\
\hline NESR & Nutrition Evidence Systematic Review \\
\hline NIH & National Institutes of Health \\
\hline P/B-24 & Pregnancy and Birth to 24 Months Project \\
\hline RCT & Randomized controlled trial \\
\hline TEC & Technical Expert Collaborative \\
\hline USDA & United States Department of Agriculture \\
\hline
\end{tabular}




\section{WHAT IS THE RELATIONSHIP BETWEEN TYPES AND AMOUNTS OF INTRODUCTION OF COMPLEMENTARY FOODS AND BEVERAGES (CFB) AND FOOD ALLERGY, ATOPIC DERMATITIS/ECZEMA, ASTHMA, AND ALLERGIC RHINITIS?}

\section{PLAIN LANGUAGE SUMMARY}

\section{What is the question?}

- The question is: What is the relationship between types and amounts of complementary foods/beverages and food allergy, atopic dermatitis/eczema, asthma, and allergic rhinitis?

What is the answer to the question?

- Peanut, tree nuts, seeds:

- Strong evidence suggests that introducing peanut in the first year of life (after 4 months of age) may reduce risk of food allergy to peanuts. This evidence is strongest for introducing peanut in infants at the highest risk (with severe atopic dermatitis and/or egg allergy) to prevent peanut allergy, but is also applicable to infants at lower risk. However, the evidence for tree nuts and sesame seeds is limited.

- Limited evidence also suggests that there is no relationship between consumption of peanut, tree nuts, or sesame seeds during the complementary feeding period and risk of atopic dermatitis/eczema and asthma.

- There is not enough evidence to determine if there is a relationship between consuming peanut, tree nuts, or seeds as complementary foods and allergic rhinitis.

- Egg:

- Moderate evidence suggests that introducing egg in the first year of life (after 4 months of age) may reduce risk of food allergy to egg.

- Limited evidence suggests that there is no relationship between the age of introduction to egg and risk of atopic dermatitis/eczema and asthma.

- There is not enough evidence to determine if there is a relationship between consuming egg as a complementary food and allergic rhinitis.

- Fish:

- Limited evidence suggests that introducing fish in the first year of life (after 4 months of age) may reduce risk of atopic dermatitis/eczema.

- There is not enough evidence to determine if there is a relationship between consuming fish as a complementary food and risk of allergy to fish or other foods, asthma, or allergic rhinitis. There is also not enough evidence to determine if there is a relationship between consuming shellfish as a complementary food and risk of food allergy, atopic dermatitis/eczema, asthma, or allergic rhinitis.

- Cow's milk products:

- Limited evidence suggests there is no relationship between age of introduction of cow's milk products, such as cheese and yogurt, and risk 
of food allergy and atopic dermatitis/eczema.

- There is not enough evidence to determine if there is a relationship between consuming milk products during the complementary feeding period and risk of asthma or allergic rhinitis.

- Wheat:

- There is not enough evidence to determine if there is a relationship between wheat consumption during the complementary feeding period and risk of food allergy, atopic dermatitis/eczema, asthma, or allergic rhinitis.

- Soy:

- There is not enough evidence to determine if there is a relationship between soybean consumption during the complementary feeding period and risk of food allergy, atopic dermatitis/eczema, asthma, or allergic rhinitis.

- Foods and beverages that are not common allergens:

- Limited evidence from observational studies suggests that introducing foods not commonly considered to be allergens, such as fruits, vegetables, and meat, in the first year of life (after 4 months of age) is not associated with risk of food allergy, atopic dermatitis/eczema, asthma, or allergic rhinitis.

- Diet diversity and dietary patterns:

- There is not enough evidence to determine a relationship between diet diversity or dietary patterns and risk of food allergy, atopic dermatitis/eczema, asthma, or allergic rhinitis.

\section{Why was this question asked?}

- This important public health question was identified and prioritized as part of the U.S. Department of Agriculture and Department of Health and Human Services Pregnancy and Birth to 24 Months Project.

\section{How was this question answered?}

- A team of Nutrition Evidence Systematic Review staff conducted a systematic review in collaboration with a group of experts called a Technical Expert Collaborative

\section{What is the population of interest?}

- Generally healthy infants and toddlers who were fed complementary foods and beverages from ages 0-24 months and had food allergy, atopic dermatitis/eczema, asthma, and allergic rhinitis examined through 18 years of age.

\section{What evidence was found?}

- This review includes studies that looked at a variety of complementary foods and beverages, including many that are major allergens:

- Peanuts, tree nuts, or seeds (14 studies)

- Eggs (28 studies)

- Fish (24 studies)

- Cow's milk products, such as cheese and yogurt (16 studies) 
- Wheat or cereals (17 studies)

- Soy (4 studies)

- Diet diversity (12 studies)

- Dietary patterns (2 studies)

- Several studies looked at foods not considered to be major allergens, such as fruits and vegetables)

- These studies looked at the age whether consuming a certain type of complementary food or beverage was related to risk of food allergy, atopic dermatitis/eczema, asthma, or allergic rhinitis.

- Complementary foods and beverages are foods and beverages other than human milk or infant formula provided to an infant or young child.

- There are limitations in the evidence as follows: use of less reliable methods to measure outcomes, only a few studies were done for some types of foods and/or outcomes, and other factors that may have had an impact on results were not always accounted for.

\section{How up-to-date is this review?}

This review includes literature from 1/1980 to 2/2017. 


\section{TECHNICAL ABSTRACT}

\section{Background}

- Complementary feeding is the process that starts when human milk or infant formula is complemented by other foods and beverages, beginning during infancy and typically continuing to 24 months of age.

- This systematic review was conducted by a team of Nutrition Evidence Systematic Review (NESR) staff as part of the U.S. Department of Agriculture and Department of Health and Human Services Pregnancy and Birth to 24 Months Project.

- The goal of this systematic review was to answer the following research question: What is the relationship between types and amounts of complementary foods/beverages and food allergy, atopic dermatitis/eczema, asthma, and allergic rhinitis?

\section{Conclusion Statement and Grade}

- Peanut, tree nuts, seeds:

- Strong evidence suggests that introducing peanut in the first year of life (after 4 months of age) may reduce risk of food allergy to peanuts. This evidence is strongest for introducing peanut in infants at the highest risk (with severe atopic dermatitis and/or egg allergy) to prevent peanut allergy, but is also applicable to infants at lower risk. However, the evidence for tree nuts and sesame seeds is limited.

- Limited evidence also suggests that there is no relationship between consumption of peanut, tree nuts, or sesame seeds during the complementary feeding period and risk of atopic dermatitis/eczema and asthma.

- There is not enough evidence to determine if there is a relationship between consuming peanut, tree nuts, or seeds as complementary foods and allergic rhinitis.

- Egg:

- Moderate evidence suggests that introducing egg in the first year of life (after 4 months of age) may reduce risk of food allergy to egg.

- Limited evidence suggests that there is no relationship between the age of introduction to egg and risk of atopic dermatitis/eczema and asthma.

- There is not enough evidence to determine if there is a relationship between consuming egg as a complementary food and allergic rhinitis.

- Fish:

- Limited evidence suggests that introducing fish in the first year of life (after 4 months of age) may reduce risk of atopic dermatitis/eczema.

- There is not enough evidence to determine if there is a relationship between consuming fish as a complementary food and risk of allergy to fish or other foods, asthma, or allergic rhinitis. There is also not enough evidence to determine if there is a relationship between consuming shellfish as a complementary food and risk of food allergy, atopic dermatitis/eczema, asthma, or allergic rhinitis. 
- Cow's milk products:

- Limited evidence suggests there is no relationship between age of introduction of cow's milk products, such as cheese and yogurt, and risk of food allergy and atopic dermatitis/eczema.

- There is not enough evidence to determine if there is a relationship between consuming milk products during the complementary feeding period and risk of asthma or allergic rhinitis.

- Wheat:

- There is not enough evidence to determine if there is a relationship between wheat consumption during the complementary feeding period and risk of food allergy, atopic dermatitis/eczema, asthma, or allergic rhinitis.

- Soy:

- There is not enough evidence to determine if there is a relationship between soybean consumption during the complementary feeding period and risk of food allergy, atopic dermatitis/eczema, asthma, or allergic rhinitis.

- Foods and beverages that are not common allergens:

- Limited evidence from observational studies suggests that introducing foods not commonly considered to be allergens, such as fruits, vegetables, and meat, in the first year of life (after 4 months of age) is not associated with risk of food allergy, atopic dermatitis/eczema, asthma, or allergic rhinitis.

- Diet diversity and dietary patterns:

- There is not enough evidence to determine a relationship between diet diversity or dietary patterns and risk of food allergy, atopic dermatitis/eczema, asthma, or allergic rhinitis.

Grade: Strong - Peanut; Moderate - Egg; Limited - Fish, cow's milk products, tree nuts or seeds, foods and beverages that are not common allergens; Grade Not Assignable - Wheat, soy, diet diversity, dietary patterns

\section{Methods}

- This systematic review was conducted by a team of NESR staff in collaboration with a Technical Expert Collaborative.

- A literature search was conducted using 4 databases (CINAHL, Cochrane, Embase, and PubMed) to identify articles published from January 1980 to February 2017 that examined the types and/or amounts of complementary foods and beverages (CFB) consumed and food allergy, atopic dermatitis/eczema, asthma, and allergic rhinitis. CFB were defined as foods and beverages other than human milk or infant formula provided to an infant or young child. Outcomes included incidence and prevalence of food allergy, atopic dermatitis/eczema, asthma, and allergic rhinitis. A manual search was done to identify articles that may not have been included in the electronic databases searched. Articles were screened in a dual manner, independently by 2 NESR analysts, to determine which articles met predetermined criteria for inclusion.

- Data from each included article were extracted, risk of bias was assessed. The 
body of evidence was qualitatively synthesized, a conclusion statement was developed and the strength of the evidence (grade) was assessed using preestablished criteria including evaluation of the internal validity/risk of bias, adequacy, consistency, impact, and generalizability of available evidence. Research recommendations were identified.

\section{Summary of Evidence}

- Thirty-nine studies included in this systematic review examined the relationship between consuming specific types of CFB (including amounts, and the age at which the specific CFB were introduced) and risk of food allergies, atopic dermatitis/eczema, asthma, and allergic rhinitis occurring during childhood through 18 years of age. An additional 12 studies examined diet diversity and two studies examined dietary patterns during the complementary feeding period in relation to these outcomes.

- A number of studies examined consumption of the most common allergenic foods during the complementary feeding period and risk of atopic disease.

- Fourteen studies examined the consumption of peanuts, tree nuts, or seeds during the complementary feeding period in relation to risk of developing atopic disease, including two RCTs. Nine studies (two RCTs) examined food allergy, five studies examined atopic dermatitis/eczema, and two studies examined asthma; no studies were identified that examined risk of allergic rhinitis.

- Twenty-eight studies examined the consumption of eggs as a complementary food in relation to risk of developing any atopic disease, including six RCTs. Thirteen studies (six RCTs) examined food allergies, fifteen studies (one RCT) examined atopic dermatitis/eczema, four studies examined asthma, and five studies examined allergic rhinitis.

- Twenty-four studies examined the consumption of fish as a complementary food in relation to risk of developing atopic disease, including one RCT. Six studies (one RCT) examined food allergies, fifteen studies examined atopic dermatitis/eczema, seven studies examined asthma, and seven studies examined allergic rhinitis.

- Sixteen studies examined the consumption of cow's milk products, such as cheese and yogurt, during the complementary feeding period in relation to risk of developing atopic disease, including one RCT. Four studies (one RCT) examined food allergies, nine studies examined atopic dermatitis/eczema, three studies examined asthma, and three studies examined allergic rhinitis.

- Eighteen studies, including 1 RCT, 11 PCSs, 5 nested case-control studies, and 1 case-control study, examined the consumption of wheat or cereals (including, but not limited to, wheat cereal) during the complementary feeding period in relation to risk of developing atopic disease. Eight studies examined food allergies, 9 studies examined atopic dermatitis/eczema, 3 studies examined asthma, and 2 studies examined allergic rhinitis.

- Four prospective cohort studies examined the relationship between age of introduction to soy and risk of developing atopic disease. One study examined food allergies, three studies examined atopic 
dermatitis/eczema, and one study examined asthma.

- A number of observational studies also examined the relationship between other types of CFB, not considered to be major allergens (e.g., fruit, vegetables, meat), and atopic diseases.

- The studies that examined diet diversity or dietary patterns were all observational, including 11 prospective cohort studies (from six cohorts) and three case-control studies.

- Many of the studies included in this review exclusively enrolled or primarily enrolled subjects who were at greater risk of allergies and/or atopic disease than the general population on the basis of family history. However, despite the inclusion of higher risk populations in this body of evidence, the results are probably generalizable to infants and toddlers who are lower risk for atopic disease but the benefit of early introduction on preventing allergy may not be as great.

- In order to better understand how specific types of foods consumed during infancy and toddlerhood impact risk of developing atopic disease, more research is needed that a) uses randomized, controlled study designs, b) uses valid and reliable measures, c) uses consistent definitions of diet diversity and/or dietary patterns, and assesses these exposures at multiple time points across the complementary feeding period, d)adjusts for key confounders, e) takes into consideration the mechanisms by which specific types of foods may affect risk of developing atopic disease when determining which diet-health relationships to investigate, and what analyses are appropriate, and f) accounts for potential for reverse causality exists due to baseline atopic disease risk status impacting both complementary feeding behaviors and risk of developing atopic disease 


\section{FULL REVIEW}

\section{Systematic review question}

What is the relationship between types and amounts of complementary foods and beverages (CFB) food allergy, atopic dermatitis/eczema, asthma, and allergic rhinitis?

\section{Conclusion statement}

Peanut, tree nuts, seeds:

- Strong evidence suggests that introducing peanut in the first year of life (after 4 months of age) may reduce risk of food allergy to peanuts. This evidence is strongest for introducing peanut in infants at the highest risk (with severe atopic dermatitis and/or egg allergy) to prevent peanut allergy, but is also applicable to infants at lower risk. However, the evidence for tree nuts and sesame seeds is limited.

- Limited evidence also suggests that there is no relationship between consumption of peanut, tree nuts, or sesame seeds during the complementary feeding period and risk of atopic dermatitis/eczema and asthma.

- There is not enough evidence to determine if there is a relationship between consuming peanut, tree nuts, or seeds as complementary foods and allergic rhinitis.

Egg:

- Moderate evidence suggests that introducing egg in the first year of life (after 4 months of age) may reduce risk of food allergy to egg.

- Limited evidence suggests that there is no relationship between the age of introduction to egg and risk of atopic dermatitis/eczema and asthma.

- There is not enough evidence to determine if there is a relationship between consuming egg as a complementary food and allergic rhinitis.

Fish:

- Limited evidence suggests that introducing fish in the first year of life (after 4 months of age) may reduce risk of atopic dermatitis/eczema.

- There is not enough evidence to determine if there is a relationship between consuming fish as a complementary food and risk of allergy to fish or other foods, asthma, or allergic rhinitis. There is also not enough evidence to determine if there is a relationship between consuming shellfish as a complementary food and risk of food allergy, atopic dermatitis/eczema, asthma, or allergic rhinitis.

Cow's milk products:

- Limited evidence suggests there is no relationship between age of introduction of cow's milk products, such as cheese and yogurt, and risk of food allergy and atopic dermatitis/eczema.

- There is not enough evidence to determine if there is a relationship between consuming milk products during the complementary feeding period and risk of asthma or allergic rhinitis.

Wheat:

- There is not enough evidence to determine if there is a relationship between wheat 
consumption during the complementary feeding period and risk of food allergy, atopic dermatitis/eczema, asthma, or allergic rhinitis.

Soy:

- There is not enough evidence to determine if there is a relationship between soybean consumption during the complementary feeding period and risk of food allergy, atopic dermatitis/eczema, asthma, or allergic rhinitis.

Foods and beverages that are not common allergens:

- Limited evidence from observational studies suggests that introducing foods not commonly considered to be allergens, such as fruits, vegetables, and meat, in the first year of life (after 4 months of age) is not associated with risk of food allergy, atopic dermatitis/eczema, asthma, or allergic rhinitis.

Diet diversity and dietary patterns:

- There is not enough evidence to determine a relationship between diet diversity or dietary patterns and risk of food allergy, atopic dermatitis/eczema, asthma, or allergic rhinitis.

\section{Grade}

Grade: Strong - Peanut; Moderate - Egg; Limited - Fish, cow's milk products, tree nuts or seeds, foods and beverages that are not common allergens; Grade Not Assignable - Wheat, soy, diet diversity, dietary patterns

\section{Summary}

- Thirty-nine studies included in this systematic review examined the relationship between consuming specific types of CFB (including amounts, and the age at which the specific CFB were introduced) and risk of food allergies, atopic dermatitis/eczema, asthma, and allergic rhinitis occurring during childhood through 18 years of age. An additional 12 studies examined diet diversity and two studies examined dietary patterns during the complementary feeding period in relation to these outcomes.

- A number of studies examined consumption of the most common allergenic foods during the complementary feeding period and risk of atopic disease.

- Fourteen studies examined the consumption of peanuts, tree nuts, or seeds during the complementary feeding period in relation to risk of developing atopic disease, including two RCTs. Nine studies (two RCTs) examined food allergy, five studies examined atopic dermatitis/eczema, and two studies examined asthma; no studies were identified that examined risk of allergic rhinitis.

- Twenty-eight studies examined the consumption of eggs as a complementary food in relation to risk of developing any atopic disease, including six RCTs. Thirteen studies (six RCTs) examined food allergies, fifteen studies (one RCT) examined atopic dermatitis/eczema, four studies examined asthma, and five studies examined allergic rhinitis.

- Twenty-four studies examined the consumption of fish as a complementary food in relation to risk of developing atopic disease, including one RCT. Six studies (one RCT) examined food allergies, 
fifteen studies examined atopic dermatitis/eczema, seven studies examined asthma, and seven studies examined allergic rhinitis.

- Sixteen studies examined the consumption of cow's milk products, such as cheese and yogurt, during the complementary feeding period in relation to risk of developing atopic disease, including one RCT. Four studies (one RCT) examined food allergies, nine studies examined atopic dermatitis/eczema, three studies examined asthma, and three studies examined allergic rhinitis.

- Eighteen studies, including 1 RCT, 11 PCSs, 5 nested case-control studies, and 1 case-control study, examined the consumption of wheat or cereals (including, but not limited to, wheat cereal) during the complementary feeding period in relation to risk of developing atopic disease. Eight studies examined food allergies, 9 studies examined atopic dermatitis/eczema, 3 studies examined asthma, and 2 studies examined allergic rhinitis.

- Four prospective cohort studies examined the relationship between age of introduction to soy and risk of developing atopic disease. One study examined food allergies, three studies examined atopic dermatitis/eczema, and one study examined asthma.

- A number of observational studies also examined the relationship between other types of CFB, not considered to be major allergens (e.g., fruit, vegetables, meat), and atopic diseases.

- The studies that examined diet diversity or dietary patterns were all observational, including 11 prospective cohort studies (from six cohorts) and three case-control studies.

- Many of the studies included in this review exclusively enrolled or primarily enrolled subjects who were at greater risk of allergies and/or atopic disease than the general population on the basis of family history. However, despite the inclusion of higher risk populations in this body of evidence, the results are probably generalizable to infants and toddlers who are lower risk for atopic disease but the benefit of early introduction on preventing allergy may not be as great.

- In order to better understand how specific types of foods consumed during infancy and toddlerhood impact risk of developing atopic disease, more research is needed that a) uses randomized, controlled study designs, b) uses valid and reliable measures, c) uses consistent definitions of diet diversity and/or dietary patterns, and assesses these exposures at multiple time points across the complementary feeding period, d)adjusts for key confounders, e) takes into consideration the mechanisms by which specific types of foods may affect risk of developing atopic disease when determining which diet-health relationships to investigate, and what analyses are appropriate, and f) accounts for potential for reverse causality exists due to baseline atopic disease risk status impacting both complementary feeding behaviors and risk of developing atopic disease

\section{Description of the evidence}

Thirty-nine studies are included in the systematic review that examined the relationship between consumption of specific types and/or amounts of CFB and risk of food allergy, 
atopic dermatitis, asthma, and allergic rhinitis occurring during childhood through $18 \mathrm{y}$ of age (Table 1). The types of CFB examined in these studies focused primarily on the most common highly allergenic foods ${ }^{\text {iv }}$, including peanut, tree nuts, seeds, eggs, fish, shellfish, cow's milk products, wheat, and soybeans. Nineteen studies examined other types of CFB, not considered to be major allergens, such as fruits, vegetables, grain products (not wheat), or meats (Table 1). In addition, 14 studies examined the relationship between diet diversity and dietary patterns during the CF period and risk of food allergy, atopic dermatitis, asthma, and allergic rhinitis occurring during childhood (Table 2).

\section{Summary of findings}

\section{Peanut, tree nuts, and seeds}

Fourteen studies examined the consumption of peanut, tree nuts, or seeds during the CF period in relation to risk of developing atopic disease, including 2 RCTs (1, 2), 6 prospective cohort studies (3-8), 4 nested case-control studies (9-12), and 2 casecontrol studies $(13,14)$ (Table 3). Two studies examined data from the Protection Against Allergy in Rural Environments Study cohort, conducted in Austria, Finland, France, Germany, Switzerland $(5,6)$, but examined different outcomes. Two studies used data from the Learning Early About Peanut Allergy Trial (LEAP) trial (1, 4), but examined different aspects of peanut consumption. Two studies examined data from the Prevalence of Infant Food Allergy, or EuroPrevall, cohort, but examined overall risk of food allergy at 2 y (10) and IgE vs non-IgE-mediated food allergy at 2 y (9).

Several studies included a subject population in which a large majority was considered to be high risk based on parental or family history of atopic disease $(1,2,4-6,8,11)$. In addition, the population examined by Du Toit et al. (1) and Greenhawt et al. (4) was also high risk on the basis of a prior diagnosis of severe atopic dermatitis (89\%) and/or egg allergy (62\%). Perkin et al. (2) enrolled exclusively BF infants from the general population, and all other studies included a mix of BF, FF, or mixed-fed infants. Most of the studies adjusted for infant milk feeding practices in analyses, though a few did not $(4,12,14)$.

The studies examined consumption of peanut, tree nuts or seeds in a variety of ways. Du Toit et al. randomized subjects to consume or avoid peanuts from 4 mo to 60 mo of age (1). Perkin et al. (2) randomized subjects to compare the effects of 'early' introduction $(3 \mathrm{mo}$ ) of 6 allergenic foods, 2 of which were peanuts and sesame, to 'standard' introduction (6 mo). Most of the remaining studies examined age of introduction to peanut, tree nuts, or sesame $(3-7,9,10,12-14)$, or the amount of peanut consumed $(2,4)$. Two studies looked at the age of introduction of several major allergens combined, including peanut and/or tree nuts $(8,11)$. Kumar et al. (11) examined egg, peanut, tree nuts, shellfish, fish, and sesame, and Zutavern et al. (8) examined soy, nuts, and chocolate.

The studies also considered various atopic disease outcomes in relation to consuming peanut, tree nuts, and seeds during the CF period, including food allergy $(1,2,4,5$, 9$12,14)$, atopic dermatitis $(3,6-8,13)$, and asthma $(1,5)$. No studies examined the

\footnotetext{
iv Food Allergen Labeling and Consumer Protection Act of 2004 (FALCPA). II, 2004.
} 
relationship between intake of peanut, tree nuts or seeds and risk of developing allergic rhinitis.

Food allergy: Nine studies examined the relationship between peanut, tree nut, and/or seed consumption and risk of developing food allergy $(1,2,4,5,9-12,14)$. Three studies assessed risk of peanut allergy specifically $(1,2,4)$, while the others examined risk of allergy to other foods associated with the intake of peanut.

Du Toit et al. (1) showed that the early peanut consumption group (at least $6 \mathrm{~g}$ of peanut protein per wk) vs. the avoidance group had a significantly reduced risk of peanut allergy, both for those who had a negative or positive $(1-4 \mathrm{~mm})$ baseline skin prick test. Greenhawt et al. (4) further analyzed data from a subgroup of the peanut consumption group, and reported that later peanut introduction during the first year of life (6-11 vs. 4-6 mo) was significantly associated with a reduced risk of peanut allergy at $5 \mathrm{y}$.

Results from the Perkin et al. (2) intention to treat analyses showed no significant differences between the standard and early introduction groups in risk of developing any food allergy from 1-3 y. However, overall adherence was poor, and only $43 \%$ of subjects complied with protocol of consuming all 6 major allergens in the specified amounts in the early introduction group. The per protocol analyses (compliers, adjusted for multiple comparisons) showed that the early introduction group had significantly lower risk of 1 or more food allergy or peanut allergy from 1-3 y. In further dose-response analyses from the early introduction group, consuming a higher weekly amount of peanut ( $2 \mathrm{~g} / \mathrm{wk}$ vs. $<2 \mathrm{~g} / \mathrm{wk}$ ) from 3 to 6 mo was significantly associated with a lower risk of peanut allergy from 1-3 $\mathrm{y}$.

Most of the associations reported in the remaining observational studies were not significant, all of which examined risk of any food allergy in relation to age of peanut or tree nuts introduction $(5,9,10,12,14)$. However, Grimshaw et al. (10) did report a trend for more food-allergic infants vs. controls to have consumed peanut and sesame before $52 \mathrm{wk}$, suggesting that the study may have been underpowered. In addition, Kumar et al. (11) found that later peanut, tree nut, and sesame introduction (>6 vs. $<6$ $\mathrm{mo}$ ) was significantly associated with lower risk of food allergy at 6-7 y; however, this was no longer significant when timing was categorized by $<1$ vs $>1 \mathrm{y}$. In addition, in children without atopic dermatitis, age of peanut, tree nut, and sesame introduction $(<6$ vs $>6 \mathrm{mo}$ ) was not associated with risk of food allergy; however, when categorized by $>1 \mathrm{vs} .<1 \mathrm{y}$, later introduction was significantly associated with lower risk of allergy at 6-7 y. Finally, in children with atopic dermatitis, age of peanut, tree nut, and sesame introduction was not significantly associated with risk of food allergy at 6-7 y. Taken together, these results may suggest potential reverse causality, whereby children at higher risk were introduced to allergenic foods later.

Atopic dermatitis: Five studies examined the relationship between age of tree nut and/or peanut introduction and risk of developing atopic dermatitis $(3,6-8,13)$. Filipiak et al. (3) found that later introduction of peanut and tree nuts ( $>6 \mathrm{vs}$. $<6 \mathrm{mo}$ ) was associated with lower risk of atopic dermatitis at $4 \mathrm{y}$. Roduit et al. (6) reported that earlier nut introduction (nut was not defined by the study authors) (3-12 vs. >12 mo) was significantly associated with decreased risk of atopic dermatitis and "atopic dermatitis with no itchy rash by 6 mo" at 1 y. Finally, Tromp et al. (7) found no significant association between age of peanut or tree nut introduction and risk of atopic 
dermatitis at 2, 3, and $4 \mathrm{y}$, and Turati et al. (13) found no significant differences between atopic dermatitis cases and controls (ages 3-24 mo) in the age of introduction to peanut, tree nuts, or cacao/chocolate. Zutavern et al. (8) found no significant associations between the age of introduction to peanut or tree nuts and risk of atopic dermatitis at $2 \mathrm{y}$ of age.

Asthma: Two studies examined the impact of tree nut/peanut consumption during the CF period and risk of asthma $(1,5)$, both reporting no significant associations. Du Toit et al. (1) found no differences in risk of asthma at 5 y between infants who were randomly assigned either to consume peanut $(6 \mathrm{~g} / \mathrm{wk})$ or to avoid consuming peanut from 4 mo to 60 mo of age. Roduit et al. (5) found that age of nut introduction (nut was not defined by the study authors) was not associated with asthma at $6 \mathrm{y}$ in a cohort study.

\section{Egg}

Twenty-eight studies examined the consumption of egg as a CFB in relation to risk of developing any atopic disease, including 6 RCTs $(2,15-19), 15$ prospective cohort studies $(3,5-8,20-29), 5$ nested case-control studies $(9-12,30)$, and 2 case-control studies $(13,14)$ (Table 4). Two studies examined subjects from the same cohort (Prevalence of Infant Food Allergy EuroPrevall) $(9,10)$. Both of these studies examined the association between age of egg introduction and food allergy risk at $2 \mathrm{y}$, but 1 reported overall risk of food allergy (10), and the other reported risk of IgEmediated food hypersensitivity (9).

A number of studies exclusively enrolled subjects who were considered to be at higher risk of atopic disease based on a prior history of atopic dermatitis earlier in infancy (16, 17 ), or because of parental or family history of atopic disease $(18,19,21,25)$. Eight additional studies included a subject population in which a majority was considered to be at higher risk based on family history of atopic disease $(2,5,6,8,11,15,23,29)$, though in most of these studies, subjects were not recruited on the basis of their risk status, and therefore are representative of risk status among the general population. Perkin et al. (2) enrolled subjects from the general population who were exclusively BF at baseline. The remaining studies included a mix of BF, FF, or mixed-fed infants. Also, most of the studies accounted for infant milk feeding practices as a potential confounder in analyses, though a few did not $(12,22)$.

The RCTs examined egg exposure in a variety of ways, and in relation to a number of different outcomes. Several RCTs compared the risk of egg allergy (15-19), among infants who were introduced to egg (at 6-12mo of age) vs. placebo during infancy. Wei-Liang Tan et al. (19) also looked at the relationship of egg introduction and atopic dermatitis. Perkin et al. (2) compared the effect of early introduction $(3 \mathrm{mo})$ of 6 allergenic foods, 1 of which was egg, to standard introduction $(6 \mathrm{mo})$. Most of the infants in the egg groups received whole-egg powder daily, either cooked $(2,16)$ or raw (17-19). In Bellach et al. (15) infants in the egg group received uncooked eggwhite powder 3 times a week. In 4 of the RCTs, the placebo group received rice powder (15, 17-19), while 1 study fed the placebo group squash powder, (16) and infants in another remained exclusively BF (2). It is also important to note that several of these RCTs were terminated early due to concerns about safety (15-17) or funding 
constraints (18).

Twenty observational studies examined the age at which egg was first consumed, and 1 examined the amount of egg consumed (22). One observational study specified that hen's egg was the exposure of interest (10), while others examined introduction of any type of egg, whether they were raw, cooked, or baked. One observational study examined several allergenic foods combined, including shellfish and fish. Kumar et al. (11) examined the age of introduction to egg, peanut, tree nuts, fish, shellfish, and sesame.

Food allergy: Six RCTs and 7 observational studies examined the relationship between consumption of egg and risk of developing food allergy $(2,5,9-12,14-19$, 24).

Four of the RCTs reported no significant effect of consuming egg vs. placebo during infancy on risk of developing egg allergy at $1 \mathrm{y}$ of age $(15,17-19)$. Three of these studies were discontinued early due to safety concerns related to high rates of egg sensitization $(15,17)$ or funding constraints $(18)$, and therefore did not enroll as many subjects as anticipated and may have been underpowered to detect a difference. Results from the other RCTs showed egg intake to be protective against development of egg allergy. Natsume et al. (16) reported that risk of hen's egg allergy at $1 \mathrm{y}$ of age was significantly higher in the placebo vs egg group (fed egg from 6-12mo of age), after which the study was terminated early due to these significant differences identified at planned interim analyses. As reported previously, Perkins et al. (2) intention to treat analyses showed no significant differences between the standard and early introduction (43\% compliance) groups in risk of developing food allergy from 1-3 $y$. The per protocol analyses showed that the early introduction group (who were fed egg in addition to 5 other allergenic foods) had significantly lower risk of 1 or more food allergies and egg allergy from 1-3 y. In further dose-response analyses from the early introduction group, consuming more egg protein ( $4 \mathrm{~g} / \mathrm{wk} \mathrm{vs}$. $<4 \mathrm{~g} / \mathrm{wk})$ from 3 to $6 \mathrm{mo}$ was significantly associated with a lower risk of egg allergy from 1-3 $\mathrm{y}$.

Results of the 8 observational studies were mixed and focused on egg introduction and the development of any food allergy, and did not specifically examine risk of egg allergy. Yu et al. (14) found that food allergy cases vs controls (ages 14-18 y) were significantly more likely to be introduced to egg earlier ( $<12$ vs. 13-24, 25-36, >36 mo). However, Yu et al. (14) did not adjust for any potential confounders, and based diagnosis of food allergy on student or parent report. Grimshaw et al. (9) found that later egg introduction (analyzed based on median age in months) was significantly associated with increased risk of any IgE-mediated food allergy at $2 \mathrm{y}$; cases with IgEmediated food allergy were introduced to egg at a mean of 52 vs 35 wk for control infants. However, Grimshaw et al. (10), using data from the same population, reported that age of hen's egg introduction (analyzed based on median in weeks) was not significantly associated with food allergy at $2 \mathrm{y}$ of age. Kumar et al. (11) found that later egg, peanut, tree nut, shellfish, fish, and sesame introduction ( $>6 \mathrm{vs} .<6 \mathrm{mo}$ ) was significantly associated with lower risk of food allergy at $\sim 6 \mathrm{y}$; however, this was no longer significant when timing was categorized by $<1 \mathrm{vs}>1 \mathrm{y}$. In addition, in children without atopic dermatitis, age of egg, peanut, tree nut, shellfish, fish, and sesame introduction ( $<6$ vs $>6 \mathrm{mo}$ ) was not significantly associated with risk of food allergy; however, when categorized by $>1$ vs. $<1$ y, later introduction was significantly 
associated with lower risk of allergy at $\sim 6 \mathrm{y}$. Finally, in children with atopic dermatitis, age of egg, peanut, tree nut, shellfish, fish, and sesame introduction was not significantly associated with risk of food allergy at $\sim 6 \mathrm{y}$. Taken together, these results may suggest potential reverse causality, whereby children at higher risk were introduced to allergenic foods later.

In addition, Hesselmar et al. (24) and Roduit et al. (5) found no significant associations between age of egg introduction and risk of any food allergy at 18 mo or $6 \mathrm{y}$, respectively. Venter et al. (12) also reported no significant differences in age of egg introduction between food allergy cases and controls at $1 \mathrm{y}$ of age.

Atopic dermatitis: One RCT (19) and 14 observational studies examined the relationship between age of introduction of egg and risk of atopic dermatitis $(3,6-8,21$ $27,29,30)$. One observational study also examined the amount of egg consumed in relation to atopic dermatitis (22). Most studies reported no significant associations.

One RCT, Wei-Liang Tan et al. (19) found no significant differences in prevalence or severity of atopic dermatitis at 8 mo or 12 mo between subjects randomized to consume raw egg powder vs. rice powder from 4 mo to 8 mo of age.

Eleven of the 14 observational studies reported no significant associations between age of introduction to egg and risk of developing atopic dermatitis at ages ranging from 18 mo to 10 y $(7,8,13,21-27,30)$.

All 3 studies that reported significant findings suggested that introducing egg later in infancy was associated with increased risk of atopic dermatitis in childhood. It is unclear whether these results may reflect reverse causality, as children with atopic dermatitis may be more likely to receive allergenic foods, like egg, later. Filipiak et al. (3) found that later egg introduction (>12 vs. $<12 \mathrm{mo}$ ) was associated with higher risk of doctor-diagnosed atopic dermatitis at $4 \mathrm{y}$. Roduit et al. (6) found that earlier egg introduction (3-12 vs. $>12 \mathrm{mo}$ ) was associated with decreased risk of atopic dermatitis at $1 \mathrm{y}$. Zutavern et al. (29) found that later egg introduction (>8 vs. $<8 \mathrm{mo}$ ) increased the risk of atopic dermatitis at $5 \mathrm{y}$.

Finally, Fergusson et al. (22) found no significant association between the amount of egg consumed at $4 \mathrm{mo}$ of age and risk of atopic dermatitis at $2 \mathrm{y}$.

Asthma: Four observational studies examined the relationship between age of egg introduction and risk of asthma, which was determined based on parent-report of doctor diagnosis $(5,23,27,28)$. Three of these studies found no significant associations between age of introduction to egg and risk of asthma $(5,23,28)$. However, Nwaru et al. (27) reported that earlier egg introduction ( $<8$ or $8-11$ vs. $>11$ $\mathrm{mo}$ ) was significantly associated with reduced risk of asthma at $5 \mathrm{y}$, specifically atopic asthma.

Allergic rhinitis: Five observational studies examined the relationship between age of egg introduction and risk of allergic rhinitis $(20,23,26-28)$. Four of the studies found no significant associations between age of introduction to egg and risk of developing allergic rhinitis $(20,23,26,28)$. However, Nwaru et al. (27) reported that earlier introduction of egg ( $<8$ or $8-11$ vs $>11 \mathrm{mo}$ ) was significantly associated with reduced risk of allergic rhinitis at $5 \mathrm{y}$. 


\section{Fish}

Twenty-four studies examined the consumption of fish as a CFB in relation to risk of developing atopic disease, including $1 \mathrm{RCT}(2), 18$ prospective cohort studies $(3,5,6$, $8,20,21,23-29,31-35), 3$ nested case-control studies $(10,11,30)$, and 2 case-control studies $(13,14)$ (Table 5). Three studies analyzed data from the Infants of Western Sweden cohort $(20,31,32)$, but examined different outcomes or age of outcomes in relation to fish consumption. Two studies examined data from the Protection Against Allergy in Rural Environments Study cohort $(5,6)$, also examining different outcomes.

Two studies exclusively enrolled subjects who were considered to be at high risk based on parental or family history of atopic disease $(21,25)$. Eight studies included a subject population where a large majority was at high risk $(2,5,6,8,11,23,29,32)$. Perkin et al. (2) enrolled subjects from the general population who were exclusively BF at baseline. The remaining studies included a mix of BF, FF, or mixed-fed infants, and all adjusted for infant milk feeding practices in analyses.

Fish consumption was examined in a variety of ways. Perkin et al. (2) compared the effect of early introduction ( $3 \mathrm{mo}$ ) of 6 allergenic foods, 1 of which was whitefish, to standard introduction (6 mo). Most of the observational studies examined age of fish introduction $(2,3,5,6,8,10,13,14,20,21,23-34)$, while others considered frequency of fish consumption $(20,31,33,35)$ or the types of fish consumed $(20,31)$ during the CF period. One observational study examined several allergenic foods combined, including shellfish and fish. Kumar et al. (11) examined the age of introduction to egg, peanut, tree nuts, shellfish, fish, and sesame.

Food allergy: One RCT (2) and 5 observational studies examined the relationship between age of fish introduction and risk of food allergy $(5,10,11,14,24)$. Three of the 6 studies found no significant associations between age of introduction and risk of any food allergy $(5,10,14)$. Most of these studies examined risk of any kind of food allergy, and did not focus on risk of fish or shellfish allergy specifically.

As has been described, while Perkins' et al. (2) intention to treat analyses showed no differences between the standard and early introduction groups in risk of developing food allergy from 1-3 y, the per protocol analyses showed that the early introduction group (who were fed fish in addition to 5 other allergenic foods) had significantly lower risk of 1 or more food allergies from 1-3 y, but no significant differences between groups in risk of developing fish allergy.

Kumar et al. (11) found that later egg, peanut, tree nut, shellfish, fish, and sesame introduction ( $>6$ vs. $<6 \mathrm{mo}$ ) was significantly associated with lower risk of any food allergy at $\sim 6 \mathrm{y}$; however, this was no longer significant when timing was categorized by $<1$ vs $>1 \mathrm{y}$. In addition, in children without atopic dermatitis, age of egg, peanut, tree nut, shellfish, fish, and sesame introduction ( $<6 \mathrm{vs}>6 \mathrm{mo}$ ) was not significantly associated with risk of food allergy; however, when categorized by $>1$ vs. $<1 \mathrm{y}$, later introduction was significantly associated with lower risk of allergy at $\sim 6 \mathrm{y}$. Finally, in children with atopic dermatitis, age of egg, peanut, tree nut, shellfish, fish, and sesame introduction was not significantly associated with risk of food allergy at $\sim 6 \mathrm{y}$. Taken together, these results may suggest potential reverse causality, whereby children at higher risk were introduced to allergenic foods later.

Finally, Hesselmar et al. (24) reported that earlier fish introduction (9 vs. 13 mo of age) 
was significantly associated with decreased risk of any food allergy at $18 \mathrm{mo}$.

Atopic dermatitis: Fifteen observational studies examined the relationship between age of introduction to fish and risk of atopic dermatitis $(3,6,8,13,21,23-27,29-31,33$, $34)$. Three studies examined frequency of fish consumption $(31,33,35)$ and 2 examined types of fish consumed $(31,35)$ in relation to risk of atopic dermatitis.

Ten of the 15 studies found no significant associations between the age at which fish was first introduced and risk of developing atopic dermatitis $(3,8,13,21,23,25-27$, $29,30)$. In these studies with null findings, the ages when atopic dermatitis were assessed ranged between 0-4 y up to $10 \mathrm{y}$ of age; most studies assessed outcomes at $4 \mathrm{y}$ of age or older.

The remaining 5 studies all reported that earlier introduction of fish was significantly associated with a decreased risk of atopic dermatitis and assessed outcomes at $4 \mathrm{y}$ of age or younger. Alm et al. (31) found that earlier fish introduction was associated with lower risk of atopic dermatitis at 12 mo (<9 vs. >9 mo; 3-5 vs. 9-12 mo; 6-8 vs. 9-12 mo). Hesselmar et al. (24) reported that earlier fish introduction was associated with decreased risk of atopic dermatitis at $18 \mathrm{mo}$ ( 8 vs. $11 \mathrm{mo}$ and 4-5/6-7 vs. 8-9 mo, 10$11 \mathrm{mo},>12 \mathrm{mo}$ ). Kull et al. (33) found that earlier fish introduction (3-8 vs. $>9 \mathrm{mo}$ ) was associated with reduced risk of atopic dermatitis at 4 y. Results from Nafstad et al. (34) showed that earlier fish introduction ( $<12 \mathrm{vs} .>12 \mathrm{mo}$ ) was associated with lower risk of atopic dermatitis at $4 \mathrm{y}$. Finally, Roduit et al. (6) reported that earlier fish introduction (3-12 vs. $>12 \mathrm{mo}$ ) was associated with decreased risk of atopic dermatitis and "atopic dermatitis with no itchy rash by 6 mo" at $1 \mathrm{y}$ of age. However, the potential for reverse causality exists, as infants at higher risk for or with symptoms of atopic dermatitis may have been introduced to allergenic foods, including fish, at older ages.

There were also a number of significant results related to frequency and/or type of fish consumed during the CF period. All 3 studies that measured frequency of fish consumption found that higher frequency of intake was significantly associated with reduced risk of atopic dermatitis in childhood between 1-4 y of age, with greatest risk reduction occurring when fish was consumed 1 or more times per week. Alm et al. (31) found that decreased frequency of fish consumption (Never vs. 3+/wk) was significantly associated with increased risk of atopic dermatitis at 12 mo. Kull et al. (33) reported that increased frequency of fish consumption at $1 \mathrm{y}$ was significantly associated with reduced risk of atopic dermatitis at $4 \mathrm{y}$ (never vs. $1 / \mathrm{mo}, 2-3 / \mathrm{mo}, 1 / \mathrm{wk}$, and $\geq 1 / w k$ ). Oien et al. (35) found that higher frequency of fish consumption ( $>1 /$ wk vs $<1 / \mathrm{wk}$ and $0 / \mathrm{wk}$ ) was significantly associated with lower risk of atopic dermatitis at $2 \mathrm{y}$, which persisted when early onset atopic dermatitis ( $<1 \mathrm{y})$ children were excluded.

In addition, Alm et al. (31) also reported that eating lean fish (e.g., cod, haddock) vs. other fish (e.g., salmon, flatfish, mackerel, and herring) was significantly associated with decreased risk of atopic dermatitis at $12 \mathrm{mo}$. While Oien et al. (35) did not make direct comparison between types of fish, results from the study showed that higher frequency of intake of both oily (redfish, halibut, salmon, trout, herring, mackerel) and lean fish (cod, coalfish) was significantly associated with lower risk of atopic dermatitis at $2 \mathrm{y}$ of age, but there were no significant findings when cod liver oil consumption was examined. Specifically, consuming oily fish more frequently ( $>1 \mathrm{vs}<1 / \mathrm{wk}, 0 / \mathrm{wk}$ ) was significantly associated with lower risk of atopic dermatitis at $2 \mathrm{y}$, which persisted when early onset atopic dermatitis ( $<1 \mathrm{y}$ ) children were excluded. Higher frequency of lean 
fish consumption ( $>1 \mathrm{vs}<1 / \mathrm{wk}, 0 / \mathrm{wk}$ ) was significantly associated with lower risk of atopic dermatitis at $2 \mathrm{y}$; however, this was no longer significant when early onset atopic dermatitis $(<1 \mathrm{y})$ children were excluded, which may suggest potential reverse causality.

Asthma: Seven studies examined the relationship between age of fish introduction and risk of developing asthma $(5,23,27,28,32-34)$, while 3 examined the relationship between frequency or type of fish consumed and asthma $(32,33,35)$. Results from these studies are mixed, and caution is used in interpreting results that may have the potential for reverse causality.

Three studies found that earlier introduction of fish, particularly when introduced before 9 mo of age, was associated with reduced risk of asthma between 4-8 y of age. Goksor et al. (32) found that earlier fish introduction ( $<9 \mathrm{vs}$. $>9 \mathrm{mo}$ ) was significantly associated with decreased risk of asthma and atopic asthma at $8 \mathrm{y}$; but not non-atopic asthma. Kull et al. (33) also reported that earlier fish introduction (3-8 vs. $>9$ mo) was significantly associated with reduced risk of asthma at $4 \mathrm{y}$. Finally, Roduit et al. (5) found that earlier fish introduction (3-12 vs. $>12 \mathrm{mo}$ ) was significantly associated with decreased risk of asthma at $6 \mathrm{y}$.

Four studies reported no significant associations between age of fish introduction and risk of asthma $(23,27,28,34)$.

There were also mixed results related to frequency and/or type of fish consumed during the CF period and risk of asthma. Goksor et al. (32) reported that frequency of fish consumption was not significantly associated with risk of asthma, atopic asthma, or non-atopic asthma at 8y. Oien et al. (35) reported that frequency of cod liver oil, fish, oily fish, or lean fish consumption was not significantly associated with risk of asthma at $2 \mathrm{y}$. However, Kull et al. (33) found that increased frequency of fish consumption at $1 \mathrm{y}$ was associated with reduced risk of asthma at $4 \mathrm{y}$ (never vs. 1/mo, 2-3/mo, 1/wk, and $\geq 1 /$ wk).

Allergic rhinitis: Seven studies examined the relationship between age of fish introduction and risk of developing allergic rhinitis $(20,23,26-28,33,34), 2$ of which also examined frequency and/or types of fish consumed in relation to allergic rhinitis risk $(20,33)$.

Five studies reported that earlier introduction of fish, particularly before 9 mo of age, was significantly associated with decreased risk of allergic rhinitis at $\sim 4-5 \mathrm{y}$ of age. Alm et al. (20) found that earlier introduction to fish ( $<9 \mathrm{vs}$. $>9 \mathrm{mo}$ ) was significantly associated with lower risk of allergic rhinitis at $4.5 \mathrm{y}$. Kull et al. (33) showed that earlier introduction of fish (3-8 vs. $>9 \mathrm{mo}$ ) was significantly associated with reduced risk of allergic rhinitis at $4 \mathrm{y}$. Nafstad et al. (34) reported that earlier fish introduction ( $<12 \mathrm{vs}$ $>12 \mathrm{mo}$ ) was significantly associated with reduced risk of allergic rhinitis at $4 \mathrm{y}$, particularly among those BF $>6 \mathrm{mo}$, with atopic dermatitis before $6 \mathrm{mo}$, and without respiratory infection $<12$ mo. Results from Nwaru et al. (27) showed that earlier introduction of fish $(6,6-9 \mathrm{vs}>9 \mathrm{mo})$ was significantly associated with reduced risk of allergic rhinitis at $5 \mathrm{y}$, with similar results when age of introduction was analyzed as a continuous variable. Finally, Virtanen et al. (28) found that earlier introduction of fish was significantly associated with decreased risk of allergic rhinitis at 5 y $(<6$ vs. $>8.5 \mathrm{mo} ; 6.1-8.5 \mathrm{vs}$. $>8.5 \mathrm{mo}$ ). However, caution should be used in interpreting the 
findings from all these studies, as there is potential for reverse causality.

Two studies reported no association between age of fish introduction and allergic rhinitis $(23,26)$.

The results related to frequency or types of fish consumption were mixed. Alm et al. (20) found no significant association between the frequency of either fish consumption or types of fish consumed from 0-12 mo and risk of allergic rhinitis at $4.5 \mathrm{y}$. However, the results from Kull et al. (33) showed that increased frequency of fish consumption at $1 \mathrm{y}$ was significantly associated with reduced risk of allergic rhinitis at $4 \mathrm{y}$ (never vs. $1 / \mathrm{mo}, 2-3 / \mathrm{mo}, 1 / \mathrm{wk}$, and $\geq 1 / \mathrm{wk}$ ).

Atopy: One study (33) examined the relationship between frequency of fish consumption during the CF period and overall risk of atopic disease, or having been diagnosed with any of the following atopic diseases: asthma, atopic dermatitis, and/or allergic rhinitis. Results showed that increased frequency of fish consumption at $1 \mathrm{y}$ was significantly associated with reduced risk of any allergic disease at 4 y (never vs. $1 / \mathrm{mo}, 2-3 / \mathrm{mo}, 1 / \mathrm{wk}$, and $\geq 1 / \mathrm{wk}$ ).

\section{Cow's milk products}

Sixteen studies examined the consumption of milk products during the CF period in relation to risk of developing atopic disease, including $1 \mathrm{RCT}(2), 11$ prospective cohort studies $(3,5,6,8,20,22,26,28,29,31,32), 2$ nested case-control studies (10, 30), and 2 case-control studies $(13,14)$ (Table 6$)$. Three studies analyzed data from the Infants of Western Sweden cohort $(20,31,32)$, but examined different outcomes or age of outcomes in relation to milk product consumption. Two studies examined data from the Protection Against Allergy in Rural Environments Study cohort $(5,6)$, but also examined different outcomes.

Six studies included a subject population where a large majority was considered high risk based on parental or family history of atopic disease $(2,5,6,8,29,32)$.

Perkin et al. (2) enrolled subjects from the general population who were exclusively $B F$. The remaining studies included a mix of BF, FF, or mixed-fed infants. And, most of the studies adjusted for infant milk feeding practices in analyses, though 1 did not (22).

Per the scope of this review, cow's milk infant formula or fluid cow's milk consumed during the first year of life were not considered to be CFB, and therefore, data related to infants' exposure to cow's milk through these products was not included.

Considered below is exposure to milk products, such as cheese and yogurt. Perkin et al. (2) compared the early introduction ( $3 \mathrm{mo}$ ) of 6 allergenic foods, 1 of which was cow's milk yogurt, to standard introduction (6 mo). Most observational studies examined age of introduction to any milk product and/or a specific type of milk product $(3,5,6,8,10,13,14,20,22,26,28-30)$. A few studies examined the type of spread used on breads (dairy vs. margarine) $(20,31,32)$ and 1 examined the amount of milk products consumed (22).

Food allergy: Four studies examined the relationship between milk product consumption and risk of food allergy $(2,5,10,14)$. Perkin et al. (2) intention to treat analyses showed no differences between the standard and early introduction groups in risk of developing food allergy from 1-3 y, but showed that the early introduction group 
(who were fed yogurt in addition to 5 other allergenic foods) had significantly lower risk of 1 or more food allergies from 1-3 y, but no significant group difference in risk of cow's milk allergy. Yu et al. (14) found that earlier introduction of dairy ( $<12$ vs. 13-24, $25-36$, and $>36 \mathrm{mo}$ ) was significantly associated with reduced risk of food allergy at 14-18 y. Conversely, Grimshaw et al. (10) found that food allergy cases were introduced to cow's milk significantly earlier than controls without food allergy (22 vs. 26 wk). However, results for age of introduction to cow's milk dairy products in any form were null. In addition, Roduit et al. (5) found that age of yogurt, other milk products, margarine, and butter introduction was not significantly associated with food allergy at $6 \mathrm{y}$.

Atopic dermatitis: Nine studies examined the relationship between milk product intake and risk of atopic dermatitis $(3,6,8,13,22,26,29-31)$. Most studies found no significant associations between the age of introduction to any milk products and atopic dermatitis $(3,6,8,13,22,26,29,30)$. Age of introduction to cheese $(13,30)$, or margarine or butter (6) was also found not to be significantly associated with atopic dermatitis. In addition, amount of dairy products consumed was not associated with atopic dermatitis at 2 y (22). However, Alm et al. (31) found that consuming margarine on bread vs. dairy spread was significantly associated with increased risk of atopic dermatitis at 12 mo., and Roduit et al. (6) reported that earlier yogurt introduction (3-12 vs. $>12 \mathrm{mo}$ ) was significantly associated with decreased risk of atopic dermatitis at $1 \mathrm{y}$.

Asthma: Three studies examined milk product consumption and risk of asthma $(5,28$, 32). Goksor et al. (32) found that toddlers (at $1 \mathrm{y}$ ) who consumed margarine or no spread vs. butter were significantly more likely to not be diagnosed with asthma at $8 \mathrm{y}$. However, Roduit et al. (5) reported no significant associations between age of yogurt, other milk product, margarine, and butter introduction and asthma at $6 \mathrm{y}$, while Virtanen et al. (28) found no significant association between age of milk product introduction and asthma at $5 \mathrm{y}$.

Allergic rhinitis: Three studies reported no significant associations between type of spread (butter vs. margarine) used (31), or age of milk product introduction $(26,28)$, and risk of allergic rhinitis.

\section{Wheat}

Eighteen studies examined the consumption of wheat during the CF period in relation to risk of developing atopic disease, including $1 \mathrm{RCT}(2), 11$ prospective cohort studies $(3,5-8,22,25,27-29,36), 5$ nested case-control studies (9-12, 30), and 1 case-control study (14) (Table 7). Two studies examined data from the Protection Against Allergy in Rural Environments Study cohort $(5,6)$, but examined different outcomes. Two studies examined data from the Prevalence of Infant Food Allergy, or EuroPrevall, cohort, but examined overall risk of food allergy at 2 y (10) and IgE vs non-IgE-mediated food allergy at 2 y (9).

One study exclusively enrolled subjects who were considered to be at high risk because of parental or family history of atopic disease (25). Eight additional studies included a subject population where a large majority was high risk $(5,6,8-11,29,36)$. Perkin et al. (2) enrolled subjects who were exclusively BF. The remaining studies included a mix of BF, FF, or mixed-fed infants. Almost all of the studies adjusted for 
infant milk feeding practices in analyses, though a few did not $(12,14,22)$.

Perkin et al. (2) compared the early introduction $(3 \mathrm{mo})$ of 6 allergenic foods, 1 of which was wheat, to standard introduction $(6 \mathrm{mo})$. All of the observational studies examined the age at which wheat was first consumed, including cereal $(3,5,6,8,13$, $22,25,29,30,36)$ or wheat or gluten $(7,9,10,12,14)$. In addition, 1 cohort study also examined the amount of cereal consumed at 4 mo of age (22).

Food allergy: One RCT and 7 observational studies examined the relationship between consumption of wheat and risk of developing food allergy, with only two examining risk of wheat allergy specifically $(2,36)$.

Perkin et al. (2) intention to treat analyses showed no differences between the standard and early introduction groups in risk of developing food allergy from 1-3 y, but showed that the early introduction group (who were fed wheat in addition to 5 other allergenic foods) had significantly lower risk of 1 or more food allergies from 1-3 y, but no significant group difference in risk of wheat allergy.

Results of the observational studies were mixed. Four studies found no significant associations between age of introduction to wheat or cereals and risk of food allergy. Grimshaw et al. (10) reported that age of wheat introduction was not associated with food allergy at $2 \mathrm{y}$, and Grimshaw et al. (9) found no significant differences in age of wheat introduction between either IgE-mediated food allergy or non-IgE-mediated food allergy cases and non-allergic controls. Venter et al. (12) found no significant differences in age of wheat introduction between food allergy cases and controls at $1 \mathrm{y}$ of age. In addition, Roduit et al. (5) found no significant association between age of cereal or bread introduction and risk of food allergy at $6 \mathrm{y}$.

Three studies found that earlier introduction to wheat was protective against development of food allergy. Poole et al. (36) reported that later introduction of cereal grains (>7 vs. 0-6 mo) was significantly associated with increased risk of wheat allergy at $4 \mathrm{y}$. Yu et al. (14) found that non-allergic controls (ages 14-18 y) were significantly more likely to be introduced to wheat/gluten earlier than cases who were diagnosed with any food allergy ( $<12$ vs. $13-24,25-36$, and $>36 \mathrm{mo}$ ). However, reverse causality cannot be ruled out in these studies. Roduit et al. (5) reported that earlier introduction of cake (3-12 vs. $>12 \mathrm{mo}$ ) was significantly associated with decreased risk of food allergy at $6 \mathrm{y}$.

Finally, Kumar et al. (11) found that later introduction of rice and wheat cereal (>6 vs. $<6 \mathrm{mo}$ ) was significantly associated with lower risk of food allergy at 6-7 $\mathrm{y}$.

Atopic dermatitis: Nine observational studies examined the relationship between consumption of wheat and risk of developing atopic dermatitis $(3,6-8,22,25,27,29$, 30).

Most studies found no significant associations between the age of introduction to wheat, cereals, biscuits, bread, or cake $(3,6-8,22,25,27,29,30)$ or amount of cereal consumed (22) and risk of atopic dermatitis. Nwaru et al. (26) found that later introduction of biscuits/bread ( $\geq 6 \mathrm{vs}$. $<6 \mathrm{mo}$ ) was significantly associated with increased risk of atopic dermatitis at $10 \mathrm{y}$ in children without atopic dermatitis by 6 mo and in children without family history of atopy.

Asthma: Three observational studies examined the relationship between age of 
introduction to grains or grain products and risk of developing asthma $(5,27,28)$. Nwaru et al. (27) found that earlier wheat cereal introduction ( $<5,5-5.5$ vs. $>5.5 \mathrm{mo})$ was significantly associated with a lower risk of asthma at $5 \mathrm{y}$. However, Roduit et al. (5) found no significant association between age of cereal, bread, or cake introduction and asthma at $6 \mathrm{y}$, and Virtanen et al. (28) reported no significant association between age of introduction to wheat and asthma at $5 \mathrm{y}$.

Allergic rhinitis: Two observational studies examined the relationship between age of introduction to grains or grain products and risk of developing allergic rhinitis $(27,28)$. Nwaru et al. (27) found that earlier introduction of wheat, rye, oat, and barley cereals $(<5,5-5.5$ vs. $>5.5 \mathrm{mo})$ was significantly associated with reduced risk of allergic rhinitis at $5 \mathrm{y}$, but no significant association with age of biscuit/bread introduction. Virtanen et al. (28) found no significant association between age of introduction to wheat and risk of allergic rhinitis at $10 \mathrm{y}$ or $5 \mathrm{y}$, respectively.

\section{Soy}

Four prospective cohort studies examined the relationship between age of introduction to soy and risk of developing atopic disease (5-8) (Table 8). All 4 studies included a subject population where the majority of subjects were considered to be at high risk of atopic disease based on parental atopy history. In addition, all 4 studies included a mix of BF, FF, or mixed-fed infants and adjusted for infant milk feeding practices in analyses. Three studies examined risk of atopic dermatitis (6-8) and 1 examined risk of food allergy and asthma (5). None of the studies reported any significant associations between the age when soy was introduced and risk of developing any of the atopic diseases assessed.

\section{CFB that are not common allergens}

A number of observational studies examined the relationship between other types of CFB, not considered to be major allergens, and atopic diseases (Table 9). Most of the studies examined age of introduction to various types of CFB, including grains other than wheat, fruits and/or vegetables, meat, chocolate, fruit syrup, orange juice, water, vitamins, and honey. In addition, 1 study examined the amount of fruit, vegetables, or meat consumed (22), and 1 examined the frequency of fermented food consumption (32).

Several studies included a subject population in which the majority of subjects were considered to be at high risk of atopic disease based on parental atopy history $(5,6,8$, $25,29,32$ ). In addition, all studies included a mix of BF, FF, or mixed-fed infants, and most adjusted for infant milk feeding practices in analyses, though a few did not (22, 37).

Grains: Seven observational studies examined the age of introduction to grains, other than wheat, and risk of atopic disease $(13,26-29,36,38)$.

One studies examined the age of introduction to grains and risk of food allergy $(5,36)$. Poole et al. (36) found no significant associations between age of introduction to rice cereal and risk of wheat allergy at $4 \mathrm{y}$. 
Five observational studies examined the relationship between consumption of grains other than wheat and risk of developing atopic dermatitis $(6,13,26,27,29,38)$. Most studies found no significant associations between the age of introduction to various grains $(6,13,26,29,30)$ and risk of atopic dermatitis. Nwaru et al. (27) found that earlier introduction of maize, rice, millet, and buckwheat cereal $(<4.5,4.5-5.5$ vs. $>5.5$ $\mathrm{mo}$ ) was significantly associated with increased risk of atopic dermatitis at $5 \mathrm{y}$. Harris et al. (38) reported that earlier rice introduction ( $<12 \mathrm{vs} .>12 \mathrm{mo}$ ) was significantly associated with higher risk of visible dermatitis at $2 \mathrm{y}$.

Two observational studies examined the relationship between age of introduction to grains other than wheat and risk of developing asthma $(5,27,28)$. Nwaru et al. (27) found that earlier rye, oat, and barley cereal introduction ( $<5,5-5.5 \mathrm{vs}$. $>5.5 \mathrm{mo})$, and Virtanen et al. (28) found that earlier oat introduction ( $<5 \mathrm{vs}$. $>5.5 \mathrm{mo} ; 5-5.5 \mathrm{vs}>5.5 \mathrm{mo}$ ) were significantly associated with a lower risk of asthma at $5 \mathrm{y}$. However, Virtanen et al. (28) reported no significant association between age of introduction to rye, barley, and other cereal (maize, rice, millet and buckwheat) and asthma at $5 \mathrm{y}$.

Two observational studies examined the relationship between age of introduction to grains other than wheat and risk of developing allergic rhinitis $(27,28)$. Nwaru et al. (27) found that earlier introduction of rye, oat, and barley cereals $(<5,5-5.5 \mathrm{vs}$. $>5.5$ mo) was significantly associated with reduced risk of allergic rhinitis at $5 \mathrm{y}$. However, the other study found no significant association between barley, rye and oat; and other cereal (maize, rice, millet and buckwheat) (28) and risk of allergic rhinitis at $10 \mathrm{y}$ or $5 \mathrm{y}$, respectively.

Fruits and/or vegetables: Fifteen observational studies examined the age of introduction to fruits and/or vegetables and risk of atopic disease $(3,5,6,8,13,22,24$ $30,35,37), 1$ of which also examined the amount of fruits and/or vegetables consumed (22).

Two studies examined age of introduction to fruits and/or vegetables and risk of IgEmediated food allergy, and neither reported significant findings. Hesselmar et al. (24) found that age of fruit introduction was not significantly associated with food allergy at $18 \mathrm{mo}$, and Roduit et al. (5) found that age of vegetable or fruit introduction was not significantly associated with food allergy at $6 \mathrm{y}$.

Twelve studies examined fruits and/or vegetables consumption and risk of atopic dermatitis. A number of studies found no significant associations between age of introduction to fruit $(3,6,8,22,24-27,29,30)$ or vegetables $(3,6,22,25-27,30)$ and risk of atopic dermatitis. In addition, Fergusson et al. (22) found no significant association between the amount of vegetable or fruit consumed at 4 mo of age, and Oien et al. (35) found no significant association between frequency of vegetable consumption, and risk of atopic dermatitis at $2 \mathrm{y}$. However, a few studies reported mixed findings in terms of which fruits and vegetables and outcomes were examined. Sariachvili et al. (30) found that atopic dermatitis cases vs. controls $(0-4 \mathrm{y})$ were significantly less often introduced to fruit juice early ( $<4 \mathrm{vs}$. $>4 \mathrm{mo}$ ). Turati et al. (13) found that earlier introduction ( $<4 \mathrm{vs}$. $>4 \mathrm{mo}$ ) of vegetables, legumes, roots, and fruit was significantly associated with decreased risk of atopic dermatitis through $24 \mathrm{mo}$. And, Zutavern et al. (8) reported that later vegetable introduction (>6 vs. 5-6 mo) was significantly associated with increased risk of doctor-diagnosed atopic dermatitis and early skin or allergic symptom atopic dermatitis at $5 \mathrm{y}$. 
Four studies examined age of introduction to fruits and/or vegetables and risk of asthma, all of which reported no significant associations $(5,27,28,37)$. One additional study reported no significant association between frequency of vegetable consumption and risk of asthma at 2 y (35).

Three studies examined age of introduction to fruits and/or vegetables and risk of allergic rhinitis, all of which reported no significant associations at either 5 y $(27,28)$ or 10 y (26).

Meat: Twelve observational studies examined the age of introduction to meat and risk of atopic disease $(3,5,6,8,13,22,25-30), 1$ of which also examined the amount of meat consumed (22).

One study examined age of meat introduction and risk of food allergy at $6 \mathrm{y}$ and reported no significant association (5).

Ten studies examined meat consumption and risk of atopic dermatitis, most of which found no significant associations between age of introduction to meat $(6,13,22,25$ $27,29,30$ ) and risk of atopic dermatitis. In addition, Fergusson et al. (22) found no significant association between the amount of meat consumed at 4 mo of age and risk of atopic dermatitis at $2 \mathrm{y}$. However, 2 studies reported that meat introduction $>6$ mo was significantly associated with increased risk at $\sim 4-5 \mathrm{y}$. Filipiak et al. (3) found that later meat introduction (>6 vs. $<6 \mathrm{mo}$ ) was significantly associated with increased risk of doctor-diagnosed atopic dermatitis at $4 \mathrm{y}$, and Zutavern et al. (8) found that later meat introduction (>6 vs. 5-6 mo) was significantly associated with increased risk of doctor-diagnosed atopic dermatitis at $5 \mathrm{y}$.

Three studies examined age of introduction to meat and risk of asthma at either 5 or 6 $y$, all of which reported no significant associations $(5,27,28)$. And, 3 studies examined age of introduction to meat and risk of allergic rhinitis at either 5 or $10 \mathrm{y}$, all of which reported no significant associations (26-28).

Other miscellaneous CFB: A few other foods and/or beverages were considered in relation to risk of developing atopic disease, the results of which are described below.

Three studies examined the age of introduction to chocolate and/or cacao, with 2 reporting that earlier introduction (3-12 vs. $>12 \mathrm{mo}$ ) was significantly associated with decreased risk of atopic dermatitis at 1 y (6) and food allergy at 6 y (5). However, Roduit et al. (5) did not find any significant associations between age of introduction to chocolate and risk of asthma at 6 y. Also, Zutavern et al. (8) found no significant associations between the age of introduction to soy, peanuts and tree nuts, and chocolate and risk of atopic dermatitis at $2 \mathrm{y}$ of age.

Strassburger et al. (37) found no significant associations between age of introduction to salty pureed foods and risk of asthma at 3-4 y of age. Andreasyan et al. (39) found no significant associations between age of fruit syrup, orange juice, water, vitamins, and honey introduction and risk of atopic dermatitis, asthma, or hay fever at $8 \mathrm{y}$ of age. Finally, Goksor et al. (32) reported that higher frequency of fermented food consumption ( $1+$ vs. 0 time/mo by age $1 \mathrm{y})$ was significantly associated with reduced risk of asthma at $8 \mathrm{y}$, but did not adjust for any potential confounders in the analysis.

\section{Diet diversity}


Twelve studies, including 11 prospective cohort studies and 1 case-control study, examined diet diversity (Table 10). Several of these studies analyzed data from the same cohorts of subjects: the Christchurch Health and Development Study (22, 4042), the Protection Against Allergy-Study in Rural Environments Study $(5,6)$, and the Lifestyle-Related Factors on the Immune System, the Development of Allergy in Childhood Study in $(8,43)$, and the German Intervention Nutritional Intervention Program $(3,44)$. The 2 remaining studies analyzed a unique data set $(13,45)$.

Diet diversity was measured using different approaches. While all of the studies determined diet diversity by summing the total number of food groups introduced by a certain age, the studies differed in terms of which food groups were considered, how diet was assessed, the categorical variables that were created, and the age at which diet diversity was assessed. Most studies examined diet diversity at 4 mo of age $(3,8$, $13,22,40-43,45)$, while others examined it at $3 \mathrm{mo}(45), 5 \mathrm{mo}(13), 6 \mathrm{mo}(3,8,44$, 45), and 12 mo (45), In addition, 2 studies used dietary assessments taken from 3 mo to 12 mo to measure cumulative diet diversity during the first year of life $(5,6)$.

In addition, studies examined several different outcomes at a range of ages (from infancy through $18 \mathrm{y}$ ), using varied diagnostic methods. Most studies examined atopic dermatitis $(3,6,8,13,22,40-45)$, with fewer examining asthma $(5,43,45)$, allergic rhinitis $(5,43,45)$, or food allergy (5). Results regarding the relationship between diet diversity during the CF period and risk of atopic disease were inconsistent.

Fergusson et al. $(22,40-42)$ reported results from the same cohort of infants (the Christchurch Health and Development Study), looking at diet diversity at $4 \mathrm{mo}$ and risk of developing atopic dermatitis later in childhood. These studies showed that greater diet diversity at 4 mo of age was associated with increased risk of atopic dermatitis later in childhood. Fergusson et al. $(22,42)$ found that greater diet diversity at 4 mo was significantly associated with increased risk of atopic dermatitis at $2 \mathrm{y}$ and $3 \mathrm{y}$. Fergusson et al. (40) and Fergusson et al. (41) both reported atopic dermatitis results at $10 \mathrm{y}$ of age, but used different analytic methods. Fergusson et al. (40) found that greater diet diversity was significantly associated with increased risk of recurrent/chronic atopic dermatitis at $10 \mathrm{y}$ using a proportional hazard model, while Fergusson et al. (41) reported a similar significant association in terms of atopic dermatitis rates per 100 subjects. All 4 studies by Fergusson et al.(22, 40-42) examined diet diversity at a single time point early in the CF period, 4 mo of age, which may not have been reflective of the diversity of the diet over the course of the CF period. In addition, risk of atopic dermatitis was based mainly on maternal report of symptoms, with few diagnoses validated by a physician. Finally, these studies did not account for a number of key confounders that could have impacted results, and were conducted in a population with rates of breastfeeding that are lower than current rates in the US.

Schoetzau et al. (44) and Filipiak et al. (3) also looked at the relationship between diet diversity and atopic dermatitis, using data from subjects enrolled in the German Infant Nutritional Intervention Program. The studies differed in terms of how diet diversity was analyzed and age at which outcomes were assessed. Schoetzau et al. (44) examined diet diversity as a continuous variable at $24 \mathrm{wk}$ of age, and examined risk of atopic dermatitis at $1 \mathrm{y}$ of age as diagnosed by a physician. Filipiak et al. (3) examined diet diversity as a categorical variable at $4 \mathrm{mo}$ and $6 \mathrm{mo}$ of age, and examined risk of 
atopic dermatitis at $4 \mathrm{y}$ as reported by parents. Both studies reported no significant associations between diet diversity and risk of atopic dermatitis. However, subjects in these studies were originally part of an RCT designed to investigate the effect of different infant formulas on risk of allergy in a high risk population compared with a non-intervention control, who were primarily at low risk of atopic disease, and it is unclear whether their risk status or varied exposure to human milk, different infant formulas, or cow's milk could have impacted outcomes.

Nwaru et al. (45) examined the relationship between diet diversity at 3, 4, 6, and 12 mo and a number of atopic disease outcomes at $5 \mathrm{y}$ of age, including atopic dermatitis, asthma (any asthma, atopic asthma, nonatopic asthma), and allergic rhinitis. These outcomes were assessed by parent report of symptoms and/or physician diagnosis. Results showed no significant associations between diet diversity at any time point and risk of atopic dermatitis or any type of asthma (i.e., atopic and nonatopic asthma combined) at $5 \mathrm{y}$. However, when types of asthma were considered separately, greater diet diversity at 4 mo was significantly associated with decreased risk of nonatopic asthma at $5 \mathrm{y}$, while lower diet diversity at 12 mo was significantly associated with increased risk of atopic asthma at $5 \mathrm{y}$. In addition, while diet diversity at $3 \mathrm{mo}$ and $4 \mathrm{mo}$ were not significantly associated with allergic rhinitis at $5 \mathrm{y}$, lower diet diversity at 6 mo and 12 mo was significantly associated with increased risk of allergic rhinitis at $5 \mathrm{y}$. However, interpretation of these results is limited due to lack of adjustment for key confounders, use of non-validated methods for assessing outcomes, and the possibility of reserve causation, particularly among at-risk children who exhibited atopic symptoms in early infancy or those with parental allergic history.

Roduit et al. $(5,6)$ examined the association of cumulative diet diversity from 3 to 12 mo of age with risk of atopic dermatitis at $1 \mathrm{y}$ and $4 \mathrm{y}(6)$ and risk of food allergy, asthma (any asthma, atopic asthma, non-atopic asthma), and allergic rhinitis (5). Results showed that lower diet diversity during the first year of life was significantly associated with increased risk of atopic dermatitis (6), and food allergy and asthma at 6 y (5). However, diet diversity was not significantly associated with risk of allergic rhinitis at 6 y (5). As with the previously discussed studies, interpretation of these results is limited due to lack of adjustment for key confounders, use of non-validated methods (e.g., parent report) for assessing outcomes, and the possibility of reserve causation, particularly among at-risk children who had parental allergic history and had allergenic food items introduced later than low risk children.

Zutavern et al. $(8,43)$ examined diet diversity at 4 and 6 mo in relation to risk of atopic dermatitis at 2 y (8) and diet diversity at 4 mo in relation to risk of atopic dermatitis, asthma, and allergic rhinitis at 6 y (43). All outcomes were determined based on parent report of symptoms and/or physician diagnosis. Zutavern et al. (8) found no significant association between diet diversity at $4 \mathrm{mo}$ and atopic dermatitis. However, greater diet diversity at 6 mo was significantly associated with decreased risk of atopic dermatitis at $2 \mathrm{y}$. In addition, Zutavern et al. (43) found no significant associations between diet diversity at 4 mo with asthma or allergic rhinitis at $6 \mathrm{y}$, but did report that greater diet diversity at 4 mo was significantly associated with increased risk of atopic dermatitis at $6 \mathrm{y}$ in children without early skin or allergic symptoms. These studies are also subject to methodological limitations, particularly the lack of adjustment for key confounders and use of non-validated methods for assessing outcomes. 
Finally, Turati et al. (13) examined diet diversity at 4 mo and 5 mo in relation to atopic dermatitis risk in a case-control study. Controls, without atopic dermatitis, had significantly greater diet diversity at 4 mo and 5 mo compared to cases (who were diagnosed with atopic dermatitis by a physician). A key limitation of this study is that the case control design introduces the possibility of recall bias, as mothers recalled infant dietary intake retrospectively.

\section{Dietary patterns}

Two studies examined dietary patterns, both of which were nested case-control studies that used data from the Prevalence of Infant Food Allergy EuroPrevall Study conducted in the United Kingdom $(9,46)$ (Table 10). Both studies examined the association between dietary patterns during the CF period and risk of food allergy at 2 y $(9,46)$. Dietary patterns were identified using principal component analysis. Grimshaw et al. (46) identified 3 dietary patterns, while Grimshaw et al. (9) identified only 1 pattern for analysis. Food allergy cases were first identified based on parental report of clinical history, blood lgE levels $\geq 0.35$, and/or skin prick test wheal $\geq 3 \mathrm{~mm}$. Infants were then placed on an exclusion diet, and those who experienced improved symptoms were then given a double-blind, placebo-controlled oral food challenge (DBPCFC) (including delayed reactions up to $48 \mathrm{hr}$ after the challenge) to determine presence of food allergy.

In both studies, consuming the "healthy dietary pattern" (high scores for 'healthy' foods (commercial baby food, toddler snacks, carrots, potatoes, bananas, lentils, broccoli) and low scores for adult foods (potato products, ready meals, sauces)) was associated with decreased risk of food allergy at $2 \mathrm{y}$. Grimshaw et al. (46) found that control infants without food allergy had significantly higher 'Infant guidelines' pattern scores than cases diagnosed with food allergy. Grimshaw et al. (9) reported that lower "healthy infant pattern" score was significantly associated with increased total risk of food allergy, IgE-mediated food allergy, and non-lgE-mediated food allergy. Grimshaw et al. (46) did not find any significant associations between the other dietary patterns identified and risk of food allergy.

\section{Evidence synthesis}

Overall, there is evidence to suggest that introducing allergenic foods in the first year of life (after $4 \mathrm{mo}$ ) does not increase risk of food allergy or atopic dermatitis, but may prevent peanut and egg allergy. The strength of the evidence depends on the specific type of CFB being considered, as well as the outcome of interest.

Strong evidence suggests that introducing peanut in the first year of life (after 4 mo of age) may reduce risk of food allergy to peanut. This evidence is strongest for introducing peanut in infants at the highest risk (with severe atopic dermatitis and/or egg allergy) to prevent peanut allergy, but is also applicable to infants at lower risk. However, the evidence for tree nuts and sesame seeds in relation to food allergy risk is limited. In addition, limited evidence also suggests that there is no relationship between consumption of peanut, tree nuts, or sesame seeds during the complementary feeding period and risk of atopic dermatitis and asthma. Finally, there 
is not enough evidence to determine if there is a relationship between consuming peanut, tree nuts, or seeds as complementary foods and allergic rhinitis.

Moderate evidence suggests that introducing egg in the first year of life (after 4 months of age) may reduce risk of food allergy to egg. Limited evidence suggests that there is no relationship between the age of introduction to egg and risk of atopic dermatitis and asthma. But, there is not enough evidence to determine if there is a relationship between consuming egg as a complementary food and allergic rhinitis. In addition, there is controversy over the forms of egg studied. Introduction of raw forms of egg (including ingestion of raw, pasteurized, dehydrated, egg white or whole-egg powder) is not recommended, as these forms may be associated with infection risk (e.g., Salmonella) and a higher risk of reactivity compared to cooked forms of egg, such as hard-boiled, scrambled, or baked. Raw forms of egg can induce more allergic reactions and more severe ones, as compared to baked and cooked forms; heating/cooking denature the egg protein, making it less allergenic than the raw form. While study subjects may not tolerate raw egg products, a majority of even egg-allergic patients can tolerate extensively heated (i.e., baked) egg-containing products (determination of baked egg tolerance in an egg-allergic patient should be done by a medical provider). The protective effects seen in the Natsume et al. (55) trial may also have been related to the smaller initial dose of $50 \mathrm{mg}$ cooked egg given first, followed by the larger dose of $250 \mathrm{mg}$, resulting in a gradual desensitization to the increased egg protein over time.

Limited evidence suggests that introducing fish in the first year of life (after 4 mo of age) may reduce risk of atopic dermatitis. However, there is not enough evidence to determine if there is a relationship between consuming fish as a complementary food and risk of allergy to fish or other foods, asthma, or allergic rhinitis. There is also not enough evidence to determine if there is a relationship between consuming shellfish (i.e., crustaceans and mollusks) as a complementary food and risk of food allergy, atopic dermatitis, asthma, or allergic rhinitis.

Limited evidence also suggests there is no relationship between age of introduction of cow's milk products, such as cheese and yogurt, and risk of food allergy and atopic dermatitis. But, there is not enough evidence to determine if there is a relationship between consuming milk products during the CF period and risk of asthma or allergic rhinitis.

A number of observational studies also examined the relationship between other types of CFB, not considered to be major allergens (e.g., fruit, vegetables, meat). Though this evidence was limited, it suggests that introducing foods not commonly considered to be allergens in the first year of life (after $4 \mathrm{mo}$ of age) is not associated with risk of food allergy, atopic dermatitis, asthma, or allergic rhinitis.

Finally, there is not enough evidence to determine if there is a relationship between wheat or soy consumption, as well as diet diversity and dietary patterns, during the CF period and risk of food allergy, atopic dermatitis, asthma, or allergic rhinitis.

Overall, the strongest conclusions were drawn for CFB and atopic disease relationships that were examined in a larger number of studies with stronger designs. Stronger conslusions were not drawn in a number of cases due to a lack of high quality evidence available to address the relationship, as the evidence was entirely, or almost 
entirely, observational in nature, including several case-controlled studies, which are prone to recall bias. In addition, many studies reported analyses that were done using data from the same cohort of subjects, or were done in small samples of individuals, or may not have been adequately powered.

The ability to draw stronger conclusions about the relationship between specific types of CFB, diet diversity, and dietary patterns and atopic disease was also restricted by a number of key methodological limitations. For example, different dietary assessment methods were used to determine what infants were consuming, and the studies considered different aspects of CFB (i.e., timing of introduction, different types consumed, the amount consumed) and examined dietary intake at different ages. In addition, studies employed different definitions of diet diversity, and typically assessed diet diversity at a single point in time, usually early in the CF period (as early as 3 to 4 months of age), and may not have reflected diversity of the diet over the entire period of complementary feeding. The 2 studies that examined dietary patterns examined the same dietary pattern in the same cohort of subjects, and it is not clear whether or not those patterns could be replicated in or are generalizable to other populations. In addition, per the scope of this review, studies that examined consumption of fluid cow's milk by infants less than 12 months $(\mathrm{mo})$ of age were not included and reviewed, and only cow's milk products, such as cheese and yogurt, were considered as CFB. Future work should address this gap by systematically reviewing studies that examined fluid cow's milk prior during the first year of life in relation to health outcomes.

Furthermore, many studies used non-validated or unreliable measures to assess risk of atopic disease, such as parent report of a physician diagnosis or the child's symptoms. Few studies based outcome assessment on a diagnosis made by a study physician using established criteria. The age at which outcomes were assessed also varied. In several studies, outcomes were measured later in childhood, when some atopic diseases, such as atopic dermatitis, may have already resolved. In other studies, outcomes were assessed early in childhood, before some diseases may have occurred.

While subjects in the studies are generalizable to the U.S. population, important confounders known to be associated with atopic disease outcomes were not always accounted for in analyses, such as consumption of human milk and/or human milk substitutes (e.g., cow's milk formula, hydrolyzed infant formula, or fluid cow's milk), parental smoking, and exposure to household pets. In addition, the potential for reverse causality also exists in this body of evidence, as baseline atopic disease risk status, based on family history or symptoms occurring in early infancy, can impact the timing and types and amounts of CFB that are introduced, as well as risk of developing various atopic diseases in early childhood. Despite a number of studies in this body of evidence that included only high-risk infants and toddlers, the results are likely applicable to lower risk populations, though potential benefits related to allergy prevention may not be as great.

\section{Research recommendations}

In order to better assess the relationship between complementary feeding and risk of 
atopic disease, additional research is needed that:

- Includes well-designed, targeted RCTs that address specific knowledge gaps

- Uses valid and reliable methods to assess infants' dietary intake and established criteria, testing, and/or biomarkers to diagnose atopic disease at ages appropriate to the typical presentation of the disease

- Adjusts for key confounders, including infant milk feeding practices (e.g., human milk, cow's milk formula, hydrolyzed infant formula, or fluid cow's milk), parental smoking, exposure to household pets, and the types of CFB introduced

- Accounts for potential reverse causality by considering subjects' baseline risk status for atopic disease

- Uses standard, consistent definitions of diet diversity, both in terms of the numbers and types of foods and food groups considered

- Considers the mechanisms by which specific types of foods, diet diversity, and dietary patterns may affect risk of developing atopic disease when determining which diet-health relationships to investigate, and what analyses are appropriate 


\section{Included articles}

1. Du T, G, Roberts, G, Sayre, P H, Bahnson, H T, Radulovic, S, et al. Randomized trial of peanut consumption in infants at risk for peanut allergy. N Engl $\mathrm{J}$ Med 2015;372:803-13.

2. Perkin, M R, Logan, K, Tseng, A, Raji, B, Ayis, S, et al. Randomized Trial of Introduction of Allergenic Foods in Breast-Fed Infants. N Engl J Med 2016;374:1733-43. 3. Filipiak, B, Zutavern, A, Koletzko, S, von B, A, Brockow, I, et al. Solid food introduction in relation to eczema: results from a four-year prospective birth cohort study. $\mathrm{J}$ Pediatr 2007;151:352-8.

4. Greenhawt, M, Fleischer, D, Chan, E S, Venter, C, Stukus, D, et al. LEAPing Through the Looking Glass: Secondary Analysis of the Effect of Skin Test Size and Age of Introduction on Peanut Tolerance after Early Peanut Introduction. Allergy 2016.

5. Roduit, C, Frei, R, Depner, M, Schaub, B, Loss, G, et al. Increased food diversity in the first year of life is inversely associated with allergic diseases. J Allergy Clin Immunol 2014;133:1056-64.

6. Roduit, C, Frei, R, Loss, G, Buchele, G, Weber, J, et al. Development of atopic dermatitis according to age of onset and association with early-life exposures. J Allergy Clin Immunol 2012;130.

7. Tromp, li, Kiefte-de J, J C, Lebon, A, Renders, C M, Jaddoe, V W, et al. The introduction of allergenic foods and the development of reported wheezing and eczema in childhood: the Generation R study. Arch Pediatr Adolesc Med 2011;165:933-8.

8. Zutavern, A, Brockow, I, Schaaf, B, Bolte, G, von B, A, et al. Timing of solid food introduction in relation to atopic dermatitis and atopic sensitization: results from a prospective birth cohort study. Pediatrics 2006;117:401-11.

9. Grimshaw, K E, Bryant, T, Oliver, E M, Martin, J, Maskell, J, et al. Incidence and risk factors for food hypersensitivity in UK infants: results from a birth cohort study. Clin Transl Allergy 2015;6:1.

10. Grimshaw, K E, Maskell, J, Oliver, E M, Morris, R C, Foote, K D, et al. Introduction of complementary foods and the relationship to food allergy. Pediatrics 2013;132.

11. Kumar, R, Caruso, D M, Arguelles, L, Kim, J S, Schroeder, A, et al. Early Life Eczema, Food Introduction, and Risk of Food Allergy in Children. Pediatr Allergy Immunol Pulmonol 2010;23:175-82.

12. Venter, C, Maslin, K, Dean, T, Arshad, S H. Does concurrent breastfeeding alongside the introduction of solid food prevent the development of food allergy? J Nutr Sci 2016;5.

13. Turati, F, Bertuccio, P, Galeone, C, Pelucchi, C, Naldi, L, et al. Early weaning is beneficial to prevent atopic dermatitis occurrence in young children. Allergy 2016;71:87888.

14. Yu Z, Day, Deborah A, Connal, Andreas, Enders, Felicity T. Early Food Allergen Exposure May Be Protective Against Food Allergies: An Extension Of The Hygiene Hypothesis. Internet Journal of Epidemiology 2011;10:1-.

15. Bellach, J, Schwarz, V, Ahrens, B, Trendelenburg, V, Aksunger, O, et al. Randomized placebo-controlled trial of hen's egg consumption for primary prevention in infants. J Allergy Clin Immunol 2016.

16. Natsume, O, Kabashima, S, Nakazato, J, Yamamoto H, K, Narita, M, et al. Twostep egg introduction for prevention of egg allergy in high-risk infants with eczema (PETIT): a randomised, double-blind, placebo-controlled trial. Lancet 2017;389:276-86. 
17. Palmer, D J, Metcalfe, J, Makrides, M, Gold, M S, Quinn, P, et al. Early regular egg exposure in infants with eczema: A randomized controlled trial. J Allergy Clin Immunol 2013;132.

18. Palmer, D J, Sullivan, T R, Gold, M S, Prescott, S L, Makrides, M. Randomized controlled trial of early regular egg intake to prevent egg allergy. J Allergy Clin Immunol 2016.

19. Wei-Liang T, J, Valerio, C, Barnes, E H, Turner, P J, Van A, P A, et al. A randomized trial of egg introduction from 4 months of age in infants at risk for egg allergy. $J$ Allergy Clin Immunol 2016.

20. Alm, B, Goksor, E, Thengilsdottir, H, Pettersson, R, Mollborg, P, et al. Early protective and risk factors for allergic rhinitis at age 4(1/2) yr. Pediatr Allergy Immunol 2011;22:398-404.

21. Bisgaard, H, Halkjaer, L B, Hinge, R, Giwercman, C, Palmer, C, et al. Risk analysis of early childhood eczema. J Allergy Clin Immunol 2009;123.

22. Fergusson, D M, Horwood, L J, Beautrais, A L, Shannon, F T, Taylor, B. Eczema and infant diet. Clin Allergy 1981;11:325-31.

23. Gustafsson, D, Sjoberg, O, Foucard, T. Development of allergies and asthma in infants and young children with atopic dermatitis--a prospective follow-up to 7 years of age. Allergy 2000;55:240-5.

24. Hesselmar, B, Saalman, R, Rudin, A, Adlerberth, I, Wold, A. Early fish introduction is associated with less eczema, but not sensitization, in infants. Acta Paediatr 2010;99:1861-7.

25. Niinivirta, K, Isolauri, E, Nermes, M, Laitinen, K. Timing of complementary feeding and the risk of atopic eczema. Acta Paediatr 2014;103:168-73.

26. Nwaru, B I, Craig, L C, Allan, K, Prabhu, N, Turner, S W, et al. Breastfeeding and introduction of complementary foods during infancy in relation to the risk of asthma and atopic diseases up to 10 years. Clin Exp Allergy 2013;43:1263-73.

27. Nwaru, B I, Takkinen, H M, Niemela, O, Kaila, M, Erkkola, M, et al. Timing of infant feeding in relation to childhood asthma and allergic diseases. J Allergy Clin Immunol 2013;131:78-86.

28. Virtanen, S M, Kaila, M, Pekkanen, J, Kenward, M G, Uusitalo, U, et al. Early introduction of oats associated with decreased risk of persistent asthma and early introduction of fish with decreased risk of allergic rhinitis. Br J Nutr 2010;103:266-73. 29. Zutavern, A, von M, E, Harris, J, Mills, P, Moffatt, S, et al. The introduction of solids in relation to asthma and eczema. Arch Dis Child 2004;89:303-8.

30. Sariachvili, M, Droste, J, Dom, S, Wieringa, M, Hagendorens, M, et al. Early exposure to solid foods and the development of eczema in children up to 4 years of age. Pediatr Allergy Immunol 2010;21:74-81.

31. Alm, B, Aberg, N, Erdes, L, Mollborg, P, Pettersson, R, et al. Early introduction of fish decreases the risk of eczema in infants. Arch Dis Child 2009;94:11-5.

32. Goksor, E, Alm, B, Pettersson, R, Mollborg, P, Erdes, L, et al. Early fish introduction and neonatal antibiotics affect the risk of asthma into school age. Pediatr Allergy Immunol 2013;24:339-44.

33. Kull, I, Bergstrom, A, Lilja, G, Pershagen, G, Wickman, M. Fish consumption during the first year of life and development of allergic diseases during childhood. Allergy 2006;61:1009-15.

34. Nafstad, P, Nystad, W, Magnus, P, Jaakkola, J J. Asthma and allergic rhinitis at 4 years of age in relation to fish consumption in infancy. J Asthma 2003;40:343-8. 
35. Oien, T, Storro, O, Johnsen, R. Do early intake of fish and fish oil protect against eczema and doctor-diagnosed asthma at 2 years of age? A cohort study. J Epidemiol Community Health 2010;64:124-9.

36. Poole, J A, Barriga, K, Leung, D Y, Hoffman, M, Eisenbarth, G S, et al. Timing of initial exposure to cereal grains and the risk of wheat allergy. Pediatrics 2006;117:2175-82.

37. Strassburger, S Z, Vitolo, M R, Bortolini, G A, Pitrez, P M, Jones, M H, et al. Nutritional errors in the first months of life and their association with asthma and atopy in preschool children. J Pediatr (Rio J) 2010;86:391-9.

38. Harris, J M, Cullinan, P, Williams, H C, Mills, P, Moffat, S, et al. Environmental associations with eczema in early life. Br J Dermatol 2001;144:795-802.

39. Andreasyan, K, Ponsonby, A L, Dwyer, T, Dear, K, Cochrane, J. Infant feeding and childhood atopy: does early introduction of non-milk fluids matter? Pediatr Allergy Immunol 2007; 18:250-7.

40. Fergusson, D M, Horwood, L J, Shannon, F T. Early solid feeding and recurrent childhood eczema: a 10-year longitudinal study. Pediatrics 1990;86:541-6.

41. Fergusson, D M, Horwood, L J. Early solid food diet and eczema in childhood: a 10year longitudinal study. Pediatr Allergy Immunol 1994;5:44-7.

42. Fergusson, D M, Horwood, L J, Shannon, F T. Risk factors in childhood eczema. J Epidemiol Community Health 1982;36:118-22.

43. Zutavern, A, Brockow, I, Schaaf, B, von B, A, Diez, U, et al. Timing of solid food introduction in relation to eczema, asthma, allergic rhinitis, and food and inhalant sensitization at the age of 6 years: results from the prospective birth cohort study LISA. Pediatrics 2008;121.

44. Schoetzau, A, Filipiak P, B, Franke, K, Koletzko, S, Von B, A, et al. Effect of exclusive breast-feeding and early solid food avoidance on the incidence of atopic dermatitis in high-risk infants at 1 year of age. Pediatr Allergy Immunol 2002;13:234-42. 45. Nwaru, B I, Takkinen, H M, Kaila, M, Erkkola, M, Ahonen, S, et al. Food diversity in infancy and the risk of childhood asthma and allergies. J Allergy Clin Immunol 2014;133:1084-91.

46. Grimshaw, K E, Maskell, J, Oliver, E M, Morris, R C, Foote, K D, et al. Diet and food allergy development during infancy: birth cohort study findings using prospective food diary data. J Allergy Clin Immunol 2014;133:511-9. 
Table 1. Description of studies that examined the relationship between consumption of specific types of complementary foods and beverages (CFB) and risk of atopic disease

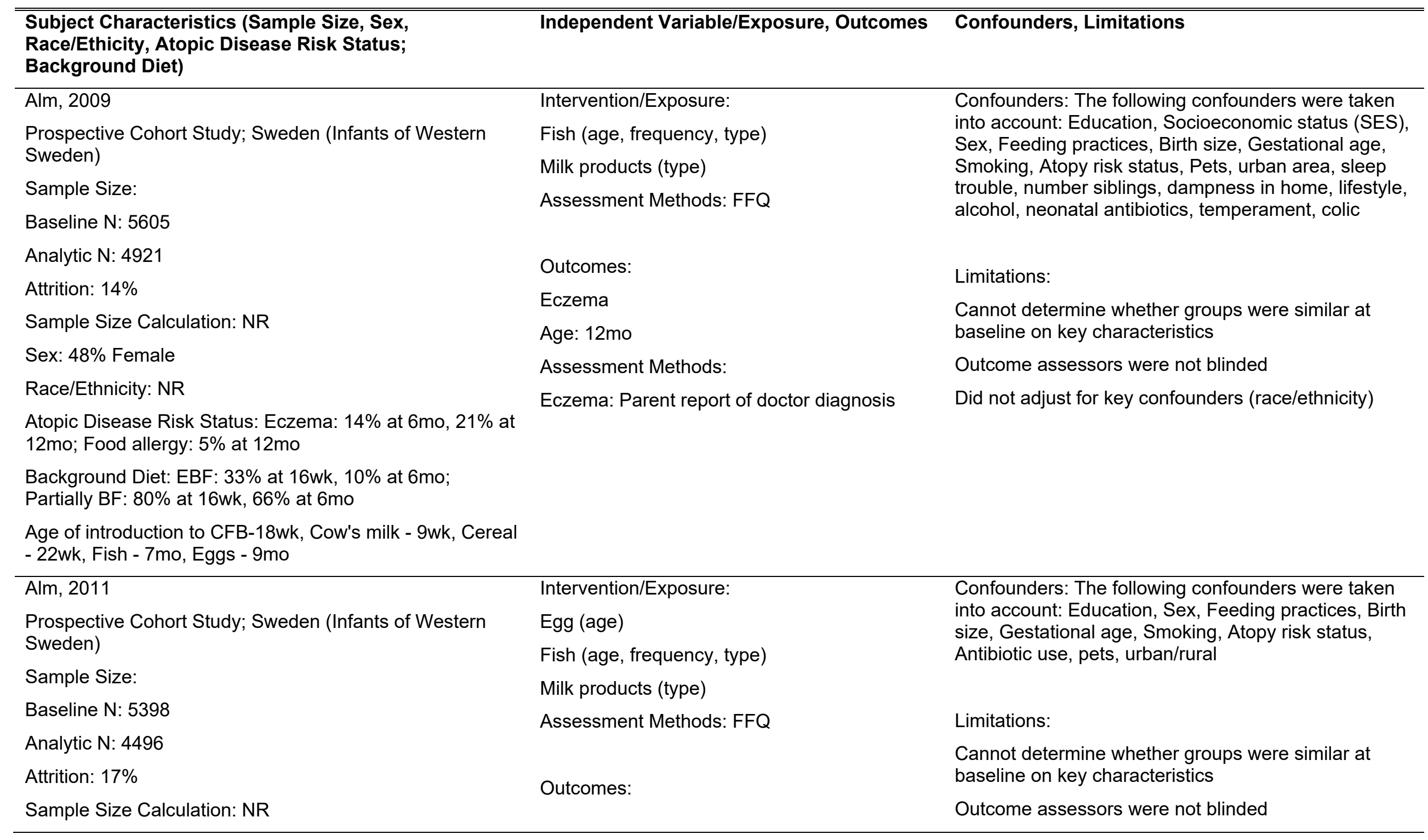




\section{Subject Characteristics (Sample Size, Sex, Race/Ethicity, Atopic Disease Risk Status; \\ Background Diet)}

\section{Sex: $48 \%$ Female}

Race/Ethnicity: NR

Atopic Disease Risk Status: NR

Background Diet: FF at 1wk: 22\%

Fish consumption: $85 \%$ more than $1 \mathrm{x} /$ mo $0-1 \mathrm{y}$ of life

Andreasyan, 2007
Prospective Cohort Study; Tasmania (Tasmanian Infant
Health Survey; Childhood Allergy and Respiratory Health Survey)

Sample Size:

Baseline N: 596

Analytic N: 499

Attrition: $16 \%$

Sample Size Calculation: $>80 \%$ power to detect $\mathrm{OR}>2$ at $\mathrm{P}<0.05$

Sex: NR

Race/Ethnicity: NR

Atopic Disease Risk Status: NR

Background Diet: NR

Bellach, 2016

Randomized Controlled Trial; Germany (Hen's Egg Allergy Prevention Study (HEAP))

Sample Size:

Egg Group Baseline N: 184

\section{Independent Variable/Exposure, Outcomes}

Confounders, Limitations

\section{Allergic rhinitis}

Age: $4.5 y$

Assessment Methods:

Allergic rhinitis: Parent report of symptoms in last $12 \mathrm{mo}$ or doctor diagnosis

Intervention/Exposure:

Other (fruit syrup, orange juice, water,

vitamins, and honey; age)

Assessment Methods: Maternal interview

\section{Outcomes:}

Eczema, Asthma, Hay fever (allergic rhinitis)

Age: 8y

Assessment Methods:

Eczema: Parent report of ever having eczema in the bends of elbows, wrists, or knees

Asthma: Parent report of whistling/wheezing in last $12 \mathrm{mo}$

Hay fever (allergic rhinitis): Parent report of sneezing, running or blocked nose, sometimes with itchy eyes or nose

Intervention/Exposure:

Egg (Egg vs. placebo from 4-6 to 12mo)

Assessment Methods: NA

\section{Attrition and sample size unclear}

Did not adjust for key confounders (race/ethnicity, SES)

Confounders: The following confounders were taken into account: Education, Sex, Feeding practices, Birth size, Gestational age, Cat/dog ownership, mold in home

\section{Limitations:}

Cannot determine whether groups were similar at baseline on key characteristics

Outcome assessors were not blinded

Attrition and sample size unclear

Did not adjust for key confounders (SES, race/ethnicity, smoking, atopy risk status)

\section{Confounders: N/A}

Limitations:

Impact of high attrition rate not assessed

Adherence was lower in the egg vs. rice group 


\section{Subject Characteristics (Sample Size, Sex, Race/Ethicity, Atopic Disease Risk Status; \\ Background Diet)}

Rice Group Baseline N: 199

Egg Group Analytic N: 124

Rice Group Analytic N: 152

Attrition: $27 \%$

Sample Size Calculation: $n=788$ total $(a=0.05, B=0.20)$ to determine a $50 \%$ reduction in sensitization to egg by $12 \mathrm{mo}$

Sex: $57 \%$ Female

Race/Ethnicity: 94\% White

Atopic Disease Risk Status: Parental allergy: 66\%

Background Diet: EBF: 35\%; Partially BF: 94\%

\section{Bisgaard, 2009}

Prospective Cohort Study; Denmark (Copenhagen Study on Asthma in Childhood)

Sample Size:

Baseline N: 411

Analytic N: 356

Attrition: $13 \%$

Sample Size Calculation: NR

Sex: $51 \%$ Female

Race/Ethnicity: NR

Atopic Disease Risk Status: Maternal asthma: 100\%

Background Diet: NR

Du Toit, 2015

Randomized Controlled Trial; United Kingdom (Learning Early About Peanut Allergy Trial (LEAP))

\section{Independent Variable/Exposure, Outcomes}

\section{Confounders, Limitations}

\section{Food allergy (egg)}

Age: $12 \mathrm{mo}$

Assessment Methods:

Food allergy: DBPCFC for egg (all subjects had hen's egg-specific $\lg \mathrm{E}<0.35 \mathrm{kU} / \mathrm{L}$ at

baseline)
Due to concerns about safety, the trial was

discontinued early

Study may have been underpowered
Intervention/Exposure:

Egg (age)

Fish (age)

Assessment Methods: NR

Outcomes:

Eczema

Age: $3 y$

Assessment Methods:

Eczema: Physician diagnosis; severity based on SCORAD
Confounders: The following confounders were taken into account: Education, SES, Sex, Feeding practices, Birth size, Smoking, Atopy risk status, Antibiotics, delivery mode

\section{Limitations:}

Cannot determine whether groups were similar at baseline on key characteristics

Did not adjust for key confounders (race/ethnicity, gestational age, pets)

All subjects at high risk (maternal asthma)

\section{Intervention/Exposure:}

Peanuts, tree nuts, or seeds (Peanut consumption vs avoidance from 4 to $6 \mathrm{mo}$ )

\section{Confounders: N/A}

Limitations: 


\section{Subject Characteristics (Sample Size, Sex, Race/Ethicity, Atopic Disease Risk Status; Background Diet)}

\section{Sample Size:}

Peanut Avoidance Group Baseline N: 321

Peanut Consumption Group Baseline N: 319

Peanut Avoidance Group Analytic N: 295

Peanut Consumption Group Analytic N: 294

\section{Attrition: 3\%}

Power Calculation: Negative SPT, power to determine a difference of $9 \%$ in avoidance vs. $2 \%$ in consumption group was $89 \%$; Positive SPT, power to determine a difference of $50 \%$ in avoidance vs. $20 \%$ in consumption group was $80 \%$

Sex: $40 \%$ female

Race/Ethnicity: 73\% White, $8 \%$ Black, 14\% Mixed, 4\% Asian, 1\% Chinese/Mid-East/ other

Atopic Disease Risk Status: Eczema, severe: 89\%; Egg allergy: $62 \%$

Background Diet: NR

\section{Fergusson, 1981}

Prospective Cohort Study; New Zealand (Christchurch Health and Development Study)

Sample Size:

Baseline N: 1262

Analytic N: 1156

Attrition: $8 \%$

Sample Size Calculation: NR

Sex: NR

\section{Independent Variable/Exposure, Outcomes}

Assessment Methods: NA

Outcomes:

Food allergy (peanut), Asthma

Age: 12mo, 30mo, 5y (60mo)

Assessment Methods:

Food allergy: OFC for peanut in subjects in whom peanut allergy was unlikely (no wheal after a SPT at $30,60 \mathrm{mo}$, no history of

allergic symptoms after peanut ingestion, no diagnosis or suspicion of allergies to sesame or tree nut, and no history of anaphylaxis in response to any food), or a DBPCFC for peanut in subjects with possible peanut allergy

Asthma: NR; categorized mild, moderate, or severe

\section{Intervention/Exposure:}

Egg (age, amount)

Milk products (age, amount)

Grain products (age, amount)

Other (fruits, vegetables, meat; age, amount)

Assessment Methods: Parent interview, food diary at $4 \mathrm{mo}$

Outcomes:

\section{Cannot determine whether groups were similar at}

baseline on key characteristics

Outcomes at $5 y$ may have been driven by sustained avoidance or sustained consumption beyond scope of CF period

Study may have been underpowered

All subjects were high risk (eczema or egg allergy)
Confounders: The following confounders were taken into account: Atopy risk status

\section{Limitations:}

Cannot determine whether groups were similar at baseline on key characteristics

Cannot determine recruitment methods, baseline characteristics, confounding factors, or blinding methods

Outcome assessors were not blinded 


\section{Subject Characteristics (Sample Size, Sex, Race/Ethicity, Atopic Disease Risk Status; \\ Background Diet)}

\section{Race/Ethnicity: NR}

Atopic Disease Risk Status: Parental atopy: 24\%

Background Diet: EBF: 19\%

\section{Filipiak, 2007}

Prospective Cohort Study; Germany (German Infant Nutritional Intervention Program)

Sample Size:

Baseline N: 5991

Analytic N: 4753

Attrition: $21 \%$

Sample Size Calculation: NR

Sex: NR

Race/Ethnicity: NR

Atopic Disease Risk Status: Familial eczema: $40 \%$ intervention, $10 \%$ control

\section{Background Diet: EBF: 51\%}

Introduction to CFB: Later, less diversity at $6 \mathrm{mo}$ in the intervention vs. control group

\section{Goksor, 2013}

Prospective Cohort Study; Sweden (Infants of Western Sweden)

Sample Size:

\section{Independent Variable/Exposure, Outcomes}

\section{Eczema}

Age: $2 y$

Assessment Methods:

Eczema: Maternal report, with some cases validated by doctor diagnosis

Intervention/Exposure:

\section{Egg (age)}

Fish (age)

Milk products (age)

Grain products (age)

Peanuts, tree nuts, or seeds (age)

Other (fruit, vegetables, meat, age)

Assessment Methods: Parent questionnaire

\section{Outcomes:}

\section{Eczema}

Age: $4 \mathrm{y}$

Assessment Methods:

Eczema: Parent report of doctor diagnosis or recurrent symptoms lasting for $6 \mathrm{mo}$ at $1 \mathrm{y}$ and 2wk at 2-4y

\section{Intervention/Exposure:}

Fish (age, frequency)

Milk products (type)

Other (fermented food; frequency)
Did not adjust for key confounders (education, SES, sex, race/ethnicity, feeding practices, birth size, gestational age, smoking, pets)

Confounders: The following confounders were taken into account: Intervention group, Education, Sex, Feeding practices, Birth size, Smoking, Atopy risk status, Sex, study region, siblings, education, smoking, birth weight

\section{Limitations:}

Cannot determine whether groups were similar at baseline on key characteristics

Outcome assessors (parent report) were not blinded

Outcomes were not assessed using valid measures (parent report of physician diagnosis)

Cannot determine attrition rates based on sample sizes presented in text/tables

Did not adjust for key confounders (SES, race/ethnicity, gestational age, pets)

Sample size calculations not reported

Confounders: The following confounders were taken into account: Education, SES, Sex, Feeding practices, Birth size, Gestational age, Smoking, Atopy risk status, Rural living, antibiotics at 1wk, outdoor activity, pets in home 


\section{Subject Characteristics (Sample Size, Sex, Race/Ethicity, Atopic Disease Risk Status; Background Diet)}

\section{Baseline N: 5044}

Analytic N: 4051

Attrition: $20 \%$

Sample Size Calculation: NR

Sex: $48 \%$ Female

Race/Ethnicity: NR

Atopic Disease Risk Status: Familial atopy: 60\%; Food allergy: 4\%; Eczema: 19\%

Background Diet: BF: $80 \%>4 \mathrm{mo}$

Fish consumption: $72 \%<9 \mathrm{mo}, 85 \% \geq 1 / \mathrm{mo}$ at $<1 \mathrm{y}$; Fermented foods: $83 \% \geq 1 / \mathrm{mo}+$ at $<1 \mathrm{y}$

\section{Greenhawt, 2016}

Prospective Cohort Study; United Kingdom (Learning Early About Peanut Allergy Trial (LEAP))

\section{Sample Size:}

Peanut Avoidance Group Baseline N: 321

Peanut Consumption Group Baseline N: 319

Peanut Avoidance Group Analytic N: 295

Peanut Consumption Group Analytic N: 294

Attrition: 3\%

Power Calculation: Negative SPT, power to determine a difference of $9 \%$ in avoidance vs. $2 \%$ in consumption group was $89 \%$; Positive SPT, power to determine a difference of $50 \%$ in avoidance vs. $20 \%$ in consumption group was $80 \%$

Sex: $40 \%$ Female

\section{Independent Variable/Exposure, Outcomes Confounders, Limitations}

Assessment Methods: FFQ

\section{Outcomes:}

Asthma, Atopic asthma, Non-atopic asthma

Age: 8y

Assessment Methods:

Asthma: Parent report of doctor diagnosis, current wheeze, or use of asthma medication

Atopic asthma: Asthma with parent report of allergic sensitization and/or doctor diagnosed rhinoconjunctivitis, food allergy, or eczema

\section{Limitations:}

Cannot determine whether groups were similar at baseline on key characteristics

Outcome assessors were not blinded

Outcome measures used were not valid/reliable

Did not adjust for key confounders (race/ethnicity)
Confounders: No confounders were accounted for

Peanuts, tree nuts, or seeds (age)

Assessment Methods: FFQ; peanut frequency questionnaire

\section{Outcomes:}

Food allergy (peanuts)

Age: $60 \mathrm{mo}(5 \mathrm{y})$

Assessment Methods:

Food allergy: for peanut in subjects in whom peanut allergy was unlikely (no wheal after a SPT at 30,60mo, no history of allergic symptoms after peanut ingestion, no

diagnosis or suspicion of allergies to sesame or tree nut, and no history of anaphylaxis in

\section{Limitations:}

Cannot determine whether groups were similar at baseline on key characteristics

Outcome assessors were not blinded

Did not adjust for key confounders (education, SES, sex, race/ethnicity, feeding practices, birth size, gestational age, smoking, atopy risk status, pets)

Did not adequately report statistical analyses, Pvalues, or data reported in figures

All subjects were high risk (eczema or egg allergy) 


\section{Subject Characteristics (Sample Size, Sex, Race/Ethicity, Atopic Disease Risk Status; \\ Background Diet)}

Race/Ethnicity: 73\% White, 8\% Black, 14\% Mixed, 4\%

Asian, 1\% Chinese/Mid-East/ other

Atopic Disease Risk Status: Eczema, severe: 89\%; Egg

allergy: $62 \%$

Background Diet: NR

Grimshaw, 2013

Nested Case-Control Study; United Kingdom (Prevalence of Infant Food Allergy EuroPrevall)

Sample Size:

Food Allergy Cases: 41

Controls: 82

Attrition: NA

Sample Size Calculation: NR

Sex: $46 \%$ Female

Race/Ethnicity: 95\% Caucasian

Atopic Disease Risk Status: Maternal asthma: 27\% cases, $13 \%$ controls; Maternal allergy: $54 \%$ cases, $38 \%$ controls

Background Diet: BF: 94\% initiated, 7wk EBF duration, 22wk duration

\section{Grimshaw, 2016}

Nested Case-Control Study; United Kingdom (Prevalence of Infant Food Allergy EuroPrevall)

Sample Size:

\section{Independent Variable/Exposure, Outcomes Confounders, Limitations}

response to any food), or a DBPCFC for

peanut in subjects with possible peanut allergy
Intervention/Exposure:

Egg (age)

Fish (age)

Milk products (age)

Grain products (age)

Peanuts, tree nuts, or seeds (age)

Assessment Methods: Food diary

\section{Outcomes:}

Food allergy

Age: $2 y$

Assessment Methods:

Food allergy: Parent report with clinical history, IgE $\geq 0.35$ and/or SPT wheal $\geq 3 \mathrm{~mm}$; exclusion diet with improved symptoms; and DBPCFC (including delayed reactions up to 48hr after the challenge)

Intervention/Exposure:

Egg (age)

Assessment Methods: Food diary
Confounders: The following confounders were taken into account: Education, Sex, Feeding practices, Smoking, Atopy risk status, Pet ownership, maternal age, single birth

\section{Limitations:}

Cannot determine whether participants were similar at baseline on key characteristics

Did not adjust for key confounders (SES, race/ethnicity, birth size, gestational age)

Small sample size, potentially underpowered
Confounders: The following confounders were taken into account: Education, Sex, Race/ethnicity, Feeding practices, Birth size, Gestational age, Smoking, Atopy risk status, Pets in home, urban 


\section{Subject Characteristics (Sample Size, Sex, Race/Ethicity, Atopic Disease Risk Status; \\ Background Diet)}

\section{Food Allergy Cases: 41}

Controls: 82

Attrition: NA

Sample Size Calculation: NR

\section{Sex: $49 \%$ Female}

Race/Ethnicity: 96\% Caucasian

Atopic Disease Risk Status: Maternal atopy: $67 \%$; Paternal atopy: 54\%; Maternal food allergy: $22 \%$; Paternal food allergy: $12 \%$

Background Diet: BF: 91\% initiated, 2.25wk EBF duration, 16wk BF duration

Gustafsson, 2000
Prospective Cohort Study; Sweden
Sample Size:
Baseline N: 100
Analytic N: 94
Attrition: $6 \%$
Sample Size Calculation: NR
Sex: NR
Race/Ethnicity: NR
Atopic Disease Risk Status: Familial atopy: $69 \%$; Familial
atopic dermatitis: $58 \%$
Background Diet: BF: 53\% <6mo; FF: $44 \%<4 \mathrm{mo}$
Hen's egg: $69 \%<12 \mathrm{mo}$; Fish: $35 \%<7 \mathrm{mo}$

\section{Independent Variable/Exposure, Outcomes Confounders, Limitations}

\section{Outcomes: \\ Food allergy}

Age: $2 y$

Assessment Methods:

Food allergy: Parent report with clinical history, IgE $\geq 0.35$ and/or SPT wheal $\geq 3 \mathrm{~mm}$; exclusion diet with imrpoved symptoms; and DBPCFC (including delayed reactions up to $48 \mathrm{hr}$ after the challenge)

\section{Limitations:}

Cannot determine whether participants were similar at baseline on key characteristics

Did not account for high loss to follow-up Did not adjust for key confounders (SES, feeding practices)

Due to concerns about reverse causality, age of egg introduction was not included in multivariate analyses Small sample size, potentially underpowered

Intervention/Exposure:
Egg (age)
Fish (age)

Assessment Methods: Parent questionnaire

\section{Outcomes:}

Atopic dermatitis, Asthma, Allergic rhinitis

Age: $4 \mathrm{mo}-8 \mathrm{y}$

Assessment Methods:

Atopic dermatitis: Parent report and clinical exam; pruritic, chronic, or chronically relapsing dermatitis

Asthma: Parent report of $\geq 3$ episodes of bronchial obstruction diagnosed by doctor
Confounders: The following confounders were taken into account: Feeding practices, Smoking, Atopy risk status, Pets in home, fever, febrile episodes

\section{Limitations:}

Cannot determine whether participants were similar at baseline on key characteristics

Cannot determine validity/reliability of measures

Cannot determine whether outcome assessors were blinded

Did not adjust for key confounders (education, SES, sex, race/ethnicity, birth size, gestational age)

All subjects were high risk (familial atopy) 


\section{Subject Characteristics (Sample Size, Sex, \\ Race/Ethicity, Atopic Disease Risk Status; \\ Background Diet)}

-

\section{Harris, 2001}

Prospective Cohort Study; United Kingdom

Sample Size:

Baseline N: 667

Analytic N: 624

Attrition: 3\%

Sample Size Calculation: NR

Sex: $46 \%$ Female

Race/Ethnicity: NR

Atopic Disease Risk Status: Familial atopy, eczema, hay fever, asthma: 26\%-38\%

Background Diet: BF: $62 \%$

\section{Hesselmar, 2010}

Prospective Cohort Study; Sweden (ALLERGY-FLORA)

Sample Size:

\section{Baseline N: 207}

Analytic N: 184

Attrition: $11 \%$

Sample Size Calculation: NR

Sex: $50 \%$ Female

Race/Ethnicity: NR

\section{Independent Variable/Exposure, Outcomes Confounders, Limitations}

Allergic rhinitis: Parent report of $\geq 2$ episodes

of rhinitis and conjunctivitis after exposure to a

particular allergen, unrelated to infection

Intervention/Exposure:

Confounders: The following confounders were taken

Grain products (age)

Assessment Methods: Parent questionnaire

\section{Outcomes:}

Atopic eczema

Age: $2 y$

Assessment Methods:

Atopic eczema: Parent report and clinical examination; itchy skin in the past 12 mo plus $\geq 3$ of following: a history of flexural

involvement, history of a generally dry skin, history of allergic disease in parents or siblings and visible dermatitis as per photographic protocol

Intervention/Exposure:

Egg (age)

Fish (age)

Other (fruit, age)

Assessment Methods: Food diary

Outcomes:

Food allergy, Eczema

Age: $18 \mathrm{mo}$ into account: Education, SES, Sex, Feeding practices,

Atopy risk status, House dust mites, crowding

\section{Limitations:}

Cannot determine whether participants were similar at baseline on key characteristics

Cannot determine validity/reliability of measures

Did not adjust for key confounders (race/ethnicity, birth size, gestational age, smoking, pets)

Confounders: The following confounders were taken

into account: Feeding practices, Gestational age,

Atopy risk status

Limitations:

Cannot determine whether participants were similar at baseline on key characteristics

Cannot determine whether outcome assessors were blinded 


\section{Subject Characteristics (Sample Size, Sex, Race/Ethicity, Atopic Disease Risk Status; \\ Background Diet)}

Atopic Disease Risk Status: Familial atopy: 49\% maternal, $41 \%$ paternal

Background Diet: BF: $88 \%$ initiated, $76 \%$ BF at $6 \mathrm{mo}, 4 \mathrm{mo}$ EBF duration, $7.5 \mathrm{mo}$ BF duration

Age of CFB introduction: Solids - 4mo, Fruit - 5mo, Hen's egg - 12mo

\section{Kull, 2006}

Prospective Cohort Study; Sweden

Sample Size:

Baseline N: 4089

Analytic N: 3619

Attrition: $12 \%$

Sample Size Calculation: NR

Sex: $49 \%$ Female

Race/Ethnicity: NR

Atopic Disease Risk Status: Familial atopy: 37\% any parent, $9 \%$ both parents; Recurrent wheeze: $4 \%$ at $1 \mathrm{y}$; Eczema: $15 \%$ at $1 \mathrm{y}$

Background Diet: BF: $20 \%<4 \mathrm{mo}$

Fish consumption at $1 \mathrm{y}: 10 \%$ never, $10 \%<1 / \mathrm{mo}, 35 \%$ $1 / \mathrm{wk}, 26 \%>1 / \mathrm{wk}, 80 \% 2-3 / \mathrm{mo}+$

\section{Kumar, 2010}

Nested Case-Control Study; United States

Sample Size:

Food Allergy Cases: 411
Independent Variable/Exposure, Outcomes

Confounders, Limitations

Assessment Methods:

Food allergy: Symptoms (immediate or lateonset reaction) after food ingestion, with OFC, and/or food-specific IgE, SPT wheal $\geq 3 \mathrm{~mm}$, and/or GI biopsy/multi-organ reactions

Eczema: Doctor diagnosis

Intervention/Exposure:

Fish (age, frequency)

Assessment Methods: Parent questionnaire

Outcomes:

Eczema, Asthma, Allergic rhinitis, Atopy

Age: 1y, 2y, 4y

Assessment Methods:

Asthma: Parent report of 4 episodes of wheeze in the past $12 \mathrm{mo}$ or 1 episode+steroid

Eczema: Parent report of dry skin and itchy rash for $>2 \mathrm{wk}$ in last $12 \mathrm{mo}$ or doctor diagnosis

Allergic rhinitis: Parent report of sneezing, running nose, red itchy eyes after exposure in the past $24 \mathrm{mo}$

Atopy: Parent report of 2+ of asthma, eczema, a/o allergic rhinitis at age $4 y$

Intervention/Exposure:

Grain products (age)

Combined allergens (egg, fish, shellfish, peanut, tree nut, sesame; age)
Did not adjust for key confounders (education, SES,

race/ethnicity, birth size, smoking, pets)

Confounders: The following confounders were taken into account: Feeding practices, Smoking, Atopy risk status, Maternal age

\section{Limitations:}

Cannot determine whether groups were similar at baseline on key characteristics

Outcome assessors were not blinded

Did not adjust for key confounders (education, SES, sex, race/ethnicity, birth size, gestational age, pets)

Confounders: The following confounders were taken into account: SES, Sex, Race/ethnicity, Feeding practices, Atopy risk status, Delivery mode, day care, birth order, pets 


\section{Subject Characteristics (Sample Size, Sex, Race/Ethicity, Atopic Disease Risk Status; Background Diet)}

Asymptomatic Sensitized Cases: 171

Controls: 378

Attrition: NA

Sample Size Calculation: NR

\section{Sex: $44 \%$ Female}

Race/Ethnicity: 86\% Caucasian

Atopic Disease Risk Status: Familial eczema: 36\%; Familial food allergy: 16\%; Familial atopy: $88 \%$

Background Diet: BF: $88 \%$ initiated, 24\% EBF

\section{Nafstad, 2003}

Prospective Cohort Study; Norway (Oslo Birth Cohort)

Sample Size:

Baseline N: 3754

Analytic N: 2531

Attrition: $34 \%$

Sample Size Calculation: NR

Sex: NR

Race/Ethnicity: NR

Atopic Disease Risk Status: NR

Background Diet: NR

\section{Independent Variable/Exposure, Outcomes}

Assessment Methods: Parent questionnaire

\section{Outcomes:}

Food allergy

Age: 6-7y

Assessment Methods:

Food allergy: Serum IgE>0.35 kUA/L; SPT, wheal $\geq 3 \mathrm{~mm}$ with typical symptoms of an allergic reaction within $2 \mathrm{hrs}$ of ingestion for egg, white, sesame, peanut, soy, milk, shrimp, walnut, cod fish, or wheat

Intervention/Exposure:

Fish (age)

Assessment Methods: Parent questionnaire

\section{Outcomes:}

Atopic dermatitis, Asthma, Allergic rhinitis

Age: $4 y$

Assessment Methods:

Atopic dermatitis: Parent report of doctor diagnosis, symptoms experienced in previous $12 \mathrm{mo}$

Asthma: Parent report of doctor diagnosis, symptoms experienced in previous $12 \mathrm{mo}$

Allergic rhinitis: Parent report of doctor diagnosis, symptoms experienced in previous $12 \mathrm{mo}$

\section{Limitations:}

Cannot determine whether groups were similar at baseline on key characteristics

Outcome assessors were not blinded

Did not adjust for key confounders (education, birth size, gestational age, smoking)

Study may have been underpowered

Confounders: The following confounders were taken into account: Education, SES, Sex, Feeding practices, Birth size, Smoking, Atopy risk status, Eczema at $6 \mathrm{mo}$, parity, maternal age, birth order, pregnancy complications, pets, respiratory infections from 0$12 \mathrm{mo}$

\section{Limitations:}

Cannot determine whether groups were similar at baseline on key characteristics

Did not use valid/reliable measures, particularly for assessing outcomes

Outcome assessors were not blinded

Did not account for high loss to follow-up

Did not adjust for key confounders (race/ethnicity, gestational age)

Study may have been underpowered 


\section{Subject Characteristics (Sample Size, Sex, Race/Ethicity, Atopic Disease Risk Status; \\ Background Diet)}

Natsume, 2017

Randomized Controlled Trial; Japan (Prevention of Egg Allergy with Tiny Amount Intake Study)

Sample Size:

Egg Group Baseline N: 74

Placebo Group Baseline N: 73

Egg Group Analytic N: 74

Placebo Group Analytic N: 73

Attrition: $18 \%$

Sample Size Calculation: $\mathrm{N}=92 /$ group to detect relative reduction of egg allergy of $65 \%$

Sex: $35 \%$ Female

Race/Ethnicity: NR

Atopic Disease Risk Status: Atopic dermatitis: 100\%; No food allergy or history of reaction to hen's egg

Background Diet: BF: $94 \%$ at $6 \mathrm{mo}, 70 \%$ at $12 \mathrm{mo}$

Age of CFB introduction: $5.6 \mathrm{mo}$

\begin{tabular}{|c|c|c|}
\hline Niinivirta, 2014 & Intervention/Exposure: & \multirow{3}{*}{$\begin{array}{l}\text { Confounders: The following confounders were taken } \\
\text { into account: Sex, Feeding practices, Gestational age, } \\
\text { Atopy risk status, Parity, animals at home, method of } \\
\text { birth }\end{array}$} \\
\hline Prospective Cohort Study; Finland & Egg (age) & \\
\hline Sample Size: & Fish (age) & \\
\hline Baseline N: 238 & Grain products (age) & \\
\hline Analytic N: 223 at $6 \mathrm{mo} ; 211$ at $12 \mathrm{mo} ; 185$ at $24 \mathrm{mo} ; 129$ at & Other (fruit, vegetables, meat; age) & Limitations: \\
\hline $\begin{array}{l}48 \mathrm{mo} \\
\text { Attrition: } 7 \% \text { at } 6 \mathrm{mo}, 11 \% \text { at } 12 \mathrm{mo}, 22 \% \text { at } 24 \mathrm{mo}, 46 \% \text { at }\end{array}$ & Assessment Methods: Food diary & $\begin{array}{l}\text { Cannot determine whether groups were similar at } \\
\text { baseline on key characteristics }\end{array}$ \\
\hline $\begin{array}{l}48 m 0 \\
\text { Sample Size Calculation: NR }\end{array}$ & Outcomes: & $\begin{array}{l}\text { Cannot determine whether outcome assessors were } \\
\text { blinded }\end{array}$ \\
\hline
\end{tabular}

\section{Independent Variable/Exposure, Outcomes Confounders, Limitations}

Intervention/Exposure:

Egg (egg vs. placebo from 6 to 12mo)

Assessment Methods: NA

\section{Outcomes:}

Food allergy

Age: 1y

Assessment Methods:

Food allergy: OFC with immediate reactions

for egg
Confounders: The following confounders were taken into account: Feeding practices, Gestational age, Atopy risk status

\section{Limitations:}

Study was terminated early because of an unexpectedly large group difference at the planned interim analysis

All subjects were high risk (atopic dermatitis) 


\section{Subject Characteristics (Sample Size, Sex, Race/Ethicity, Atopic Disease Risk Status; \\ Background Diet)}

\section{Sex: $48 \%$ Female}

Race/Ethnicity: NR

Atopic Disease Risk Status: Familial atopy: 100\%

Background Diet: BF: $73 \%>6 \mathrm{mo}, 3.4 \mathrm{mo}$ EBF duration, 8.3mo BF duration

\section{Independent Variable/Exposure, Outcomes}

Atopic eczema

Age: $6 \mathrm{mo}, 1 \mathrm{y}, 2 \mathrm{y}, 4 \mathrm{y}$

Assessment Methods:

Atopic eczema: Doctor diagnosed eczema, based on confirmation with pruritus, facial and/or extensor involvement and chronic course (>1mo); SPT wheal $>3 \mathrm{~mm}$ for cow's milk, raw hen's egg white, wheat and rice

flour, gliadin, cod, soya bean, birch, six grasses, cat, dog, Dermatophagoides pteronyssimus allergen, latex, and potato, carrot and banana

Nwaru, 2013a

Prospective Cohort Study; Finland (Finnish Type 1 Diabetes Prediction and Prevention Study)

Sample Size:

Baseline N: 4075

Analytic N: 3781

Attrition: $7 \%$

Sample Size Calculation: NR

\section{Sex: $47 \%$ Female}

Race/Ethnicity: NR

Atopic Disease Risk Status: Parental asthma: 13\%; Parental allergic rhinitis: $52 \%$

Background Diet: BF: 1.4mo EBF duration, 7mo BF duration

Intervention/Exposure:

Egg (age)

Fish (age)

Grain products (age)

Other (fruit, vegetables, meat; age)

Assessment Methods: Parent questionnaire

Outcomes:

Atopic eczema, Asthma, Allergic rhinitis

Age: $5 y$

Assessment Methods:

Atopic eczema: Parent report of doctor diagnosis

Asthma: Parent report of doctor diagnosis, with any wheezing symptom or use of asthma medication during last $12 \mathrm{mo}$
Did not account for high loss to follow-up

Did not adjust for key confounders (education, SES, race/ethnicity, birth size, smoking)

Only reported cumulative incidence at $4 y$, and not incidence at other time points measured $(6 \mathrm{mo}, 1 \mathrm{y}$, or 2y)

All subjects were high risk (familial atopy)
Confounders: The following confounders were taken into account: Education, Sex, Feeding practices, Birth size, Gestational age, Smoking, Atopy risk status, Number of siblings, hospital of birth, season of birth, maternal age, pets at home, mode of delivery

\section{Limitations:}

Cannot determine whether groups were similar at baseline on key characteristics

Outcome assessors were not blinded

Did not adjust for key confounders (SES, race/ethnicity)

Did not show adjusted data, or data when CFB were analyzed as continuous variables 


\section{Subject Characteristics (Sample Size, Sex, Race/Ethicity, Atopic Disease Risk Status; Background Diet)}

CFB introduction: Cow's milk at $1.8 \mathrm{mo}$, followed by roots, fruits and berries, oats, wheat, rye, barley, meat, fish, and egg

\begin{tabular}{ll}
\hline Nwaru, 2013b & Intervention/Exposure: \\
Prospective Cohort Study; United Kingdom (Study of & Egg (age) \\
Eczema and Asthma To Observe the Influence of & Fish (age) \\
Nutrition) & Milk products (age) \\
Sample Size: & Grain products (age) \\
Baseline N: 1924 & Other (fruit, vegetables, meat; age) \\
Analytic N: 934 & Assessment Methods: Maternal questionnaire \\
Attrition: $51 \%$ &
\end{tabular}

Sample Size Calculation: NR

Sex: $50 \%$ Female

Race/Ethnicity: NR

Atopic Disease Risk Status: Maternal atopy: 41\%

Background Diet: BF: $31 \%<2.25 \mathrm{mo}, 51 \%$ EBF <3.75mo; FF: $81 \%$

\section{Oien, 2010}

Prospective Cohort Study; Norway (Prevention of Allergy Among Children in Trondheim Study)

Sample Size:

Baseline N: 5171

Analytic N: 3086

Attrition: $40 \%$

\section{Independent Variable/Exposure, Outcomes Confounders, Limitations}

Allergic rhinitis: Parent report of sneezing,

nasal congestion, or rhinitis other than with

respiratory tract infections accompanied by

itching of the eye and tearing during last $12 \mathrm{mo}$

Confounders: The following confounders were taken

into account: Education, SES, Sex, Feeding practices, Birth size, Gestational age, Smoking, Atopy risk

status, Birth order, maternal age, use if antibiotics by $1 \mathrm{y}$

Limitations:

Cannot determine whether groups were similar at baseline on key characteristics

Outcome assessors were not blinded

Outcomes:

Eczema, Hay fever (allergic rhinitis)

Age: $10 y$

Assessment Methods:

Eczema: Parent report of doctor diagnosis

Hay fever (allergic rhinitis): Parent report of doctor diagnosis

Intervention/Exposure:

Fish (frequency/type)

Other (vegetables; frequency)

Assessment Methods: FFQ

Outcomes:
Confounders: The following confounders were taken
Groups differed at baseline in terms of maternal

education, but this was not adjusted for in analyses
Did not adjust for key confounders (race/ethnicity, pets)

Did not use valid/reliable measures (parent report of outcomes)

Did not account for high loss to follow-up into account: SES, Sex, Feeding practices, Birth size, Smoking, Atopy risk status

Limitations: 


\section{Subject Characteristics (Sample Size, Sex, \\ Race/Ethicity, Atopic Disease Risk Status; \\ Background Diet)}

Sample Size Calculation: NR

Sex: $51 \%$ Female

Race/Ethnicity: NR

Atopic Disease Risk Status: Familial atopy: 35\% (one family member), 26\% (two), 6\% (three)

Background Diet: BF: $74 \%$ EBF >4mo

Palmer, 2013

Randomized Controlled Trial; Australia

Sample Size:

Egg Group Baseline N: 49

Rice Group Baseline N: 37

Egg group Analytic N: 42

Rice Group Analytic N: 35

Attrition: 10\%

Sample Size Calculation: N=103/group to detect a $20 \%$ risk reduction

\section{Sex: $34 \%$ Female}

Race/Ethnicity: 79\% White (maternal race)

Atopic Disease Risk Status: Eczema, moderate to severe:

100\%; Familial atopy: $>90 \%$

Background Diet: BF: $99 \%$ initiated, $84 \%$ at $4 \mathrm{mo}$

No CFB introduction ( $<4 \mathrm{mo}$ ) or experience ingesting egg

\section{Independent Variable/Exposure, Outcomes Confounders, Limitations}

\section{Eczema, Asthma}

Age: $2 y$

Assessment Methods:

Eczema: Parent report of ever having eczema or itchy rash for at least $6 \mathrm{mo}$

Asthma: Parent report of doctor diagnosis

Intervention/Exposure:

Egg (Egg vs. placebo from 4-6 to 10mo)

Assessment Methods: NA

Outcomes:

Food allergy (egg)

Age: $8 \mathrm{mo}, 12 \mathrm{mo}$

Assessment Methods:

Food allergy: OFC and positive SPT for egg

\section{Outcome assessors were not blinded}

Did not adjust for key confounders (education,

race/ethnicity, gestational age, pets)

Did not use valid/reliable measures (parent report of outcomes)

Did not account for high loss to follow-up

Only conducted multivariate analyses for variables that were significant with univariate analyses

Confounders: N/A

Limitations:

$78 \%$ and $64 \%$ of subjects did not follow advice to consume an egg-free diet (no difference between groups)

Due to concerns about safety, the trial was discontinued early

Study may have been underpowered

All subjects were high risk (eczema) 


\section{Subject Characteristics (Sample Size, Sex, \\ Race/Ethicity, Atopic Disease Risk Status; \\ Background Diet)}

Palmer, 2016

Randomized Controlled Trial; Australia (Starting Time of Egg Protein Trial (STEP))

Sample Size:

Egg Group Baseline N: 407

Egg-free Group Baseline N: 413

Egg Group Analytic N: 371

Egg-free Group Analytic N: 377

Attrition: $9 \%$

Sample Size Calculation: N=597/group to detect a 50\% risk reduction; final sample size provided $68 \%$ power to detect a $50 \%$ risk reduction

\section{Sex: $51 \%$ Female}

Race/Ethnicity: 89\% White (maternal race)

Atopic Disease Risk Status: Maternal atopy: 100\%;

Eczema: 0\%

Background Diet: BF: $99 \%$ initiated, $66 \%$ at $4 \mathrm{mo}$

No CFB introduction (<4mo) or experience ingesting egg

\section{Perkin, 2016}

Randomized Controlled Trial and Prospective Cohort Study; United Kingdom (Enquiring about Tolerance Trial (EAT))

Sample Size:

Standard Introduction Group Baseline N: 651

Early Introduction Group Baseline N: 652

Standard Introduction Group Analytic N: 595

\section{Independent Variable/Exposure, Outcomes}

Intervention/Exposure:

Egg (Egg vs. placebo from 4-6 to 10mo)

Assessment Methods: NA

Outcomes:

Food allergy (egg)

Age: $12 \mathrm{mo}$

Assessment Methods:

Food allergy: OFC and positive SPT for egg
Confounders: The following confounders were taken into account: Sex, Feeding practices, Birth size, Gestational age, Atopy risk status, City

Limitations:

Subjects could introduce eggs beginning at $10 \mathrm{mo}$, but outcomes weren't assessed until 12mo (and egg intake from 10 to 12 mo was not reported or accounted for)

Study was underpowered (68\% power; study recruitment ended due to funding constraints)

All subjects were high risk (maternal atopy)

\section{Intervention/Exposure:}

Egg (amount)

Peanuts, tree nuts, or seeds (amount)

Combined allergens (Early introduction (3mo) of cow's milk (yogurt), peanut, cooked (boiled) hen's egg, sesame, and whitefish, and wheat vs late $(6 \mathrm{mo}))$

Assessment Methods: NA

\section{Confounders: N/A}

Limitations:

C-section rate differed between groups at baseline, and was not adjusted for

Adherence to the study protocol was lower in the early introduction group

Participants were not blinded to their intervention group; Cannot determine whether investigators were blinded 


\section{Subject Characteristics (Sample Size, Sex, Race/Ethicity, Atopic Disease Risk Status; \\ Background Diet)}

\section{Early Introduction Group Analytic N: 567}

\section{Attrition: $11 \%$}

Sample Size Calculation:80\% power at the 5\%

significance level to detect $50 \%$ reduction in prevalence of food allergy

\section{Sex: $49 \%$ Female}

Race/Ethnicity: 85\% White, 3\% Black, 2\% Asian, 1\% Chinese, 9\% Mixed

Atopic Disease Risk Status: Parental atopy: 82\%; Eczema: 24\%

Background Diet: BF: 100\% EBF

\section{Poole, 2006}

Prospective Cohort Study; United States (Diabetes Autoimmunity Study in the Young)

Sample Size:

Baseline N: 1819

Analytic N: 1612

Attrition: 11\%

Sample Size Calculation: NR

Sex: $31 \%$ Female

Race/Ethnicity: 70\% non-Hispanic White, 23\% Hispanic, $4 \%$ Biracial, $2 \%$ Black

Atopic Disease Risk Status: Familial atopy: 63\%

Background Diet: BF: 10.3mo duration

CFB introduction: $75 \%$ introduced to cereal grains $>7 \mathrm{mo}$, $75 \%$ introduced to rice $<6 \mathrm{mo}$

Outcomes:

Food allergy (cow's milk, peanut, egg,

sesame, fish, or wheat)

Age: 1-3y

Assessment Methods:

Food allergy: Positive SPT and DBPCFC for cow's milk, peanut, egg, sesame, fish, or wheat

Intervention/Exposure:

Grain products (age)

Assessment Methods: Parent questionnaire

\section{Outcomes:}

Food allergy (wheat)

Age: 3, 6, 9, 12, 15mo, 2y, 3y, 4y

Assessment Methods:

Food allergy: Parent report of doctor diagnosis of wheat allergy
Confounders: The following confounders were taken into account: Feeding practices, Atopy risk status

\section{Limitations:}

Cannot determine whether groups were similar at baseline on key characteristics

Outcome assessors were not blinded

Did not adjust for key confounders (SES, sex, education, race/ethnicity, birth size, gestational age, smoking, pets)

Did not use valid/reliable measures (parent report of outcomes) 


\section{Subject Characteristics (Sample Size, Sex, Race/Ethicity, Atopic Disease Risk Status; \\ Background Diet)}

Roduit, 2012

Prospective Cohort Study; Austria, Finland, France, Germany, Switzerland (Protection Against Allergy in Rural Environments Study)

Sample Size:

Baseline N: 1133

Analytic N: 1041

Attrition: $8 \%$

Sample Size Calculation: NR

Sex: NR

Race/Ethnicity: NR

Atopic Disease Risk Status: Parental allergy: 54\%

Background Diet: BF: $66 \%$ EBF at $2 \mathrm{mo}, 46 \%>6 \mathrm{mo}$

CFB introduction: $2 \%$ received no CFB by $12 \mathrm{mo}$; Among children with allergic parents, dairy products, egg, nut, and soy were introduced later

Roduit, 2014
Prospective Cohort Study; Austria, Finland, France,
Germany, Switzerland (Protection Against Allergy in Rural
Environments Study)

Sample Size:

Baseline N: 1133

Analytic N: 856

Attrition: $24 \%$

\section{Independent Variable/Exposure, Outcomes}

Confounders, Limitations

Intervention/Exposure:

Egg (age)

Fish (age)

Milk products (age)

Grain products (age)

Peanuts, tree nuts, or seeds (age)

Soybeans (age)

Other (fruit, vegetables, meat, chocolate; age)

Assessment Methods: Food diary

\section{Outcomes:}

Atopic dermatitis

Age: 1y, 4y

Assessment Methods:

Atopic dermatitis: Parent report of doctor diagnosis at least once between $12 \mathrm{mo}$ and $4 \mathrm{y}$, or medical exam with positive SCORAD scores $(>0)$ assessed at $1 \mathrm{y}$, or both

\section{Intervention/Exposure:}

Egg (age)

Fish (age)

Milk products (age)

Grain products (age)

Peanuts, tree nuts, or seeds (age)

Soybeans (age)
Confounders: The following confounders were taken into account: Education, Feeding practices, Smoking Atopy risk status, Study center, farming

Limitations:

Cannot determine whether groups were similar at baseline on key characteristics

Outcome assessors were not blinded

Did not use valid/reliable measures (parent reported outcomes)

Did not adjust for key confounders (SES, sex, race/ethnicity, birth size, gestational age, pets)

Did not report statistical results of analyses shown in figures 


\section{Subject Characteristics (Sample Size, Sex, \\ Race/Ethicity, Atopic Disease Risk Status; \\ Background Diet)}

\section{Sample Size Calculation: NR}

Sex: $50 \%$ Female

Race/Ethnicity: NR

Atopic Disease Risk Status: Parental allergy: 54\%

Background Diet: BF: $91 \%$ initiated, 26\% >10mo
Independent Variable/Exposure, Outcomes

Other (fruit, vegetables, meat, chocolate; age)

Assessment Methods: Food diary

\section{Outcomes:}

Food allergy, Asthma

Age: $6 y$

Assessment Methods:

Food allergy: Parent report of doctor diagnosis

Asthma: Parents report of doctor diagnosis or at least 2 doctor-diagnosed episodes of obstructive bronchitis in the last $12 \mathrm{mo}$

Allergic rhinitis: Parent report of itchy, runny, or blocked nose without a cold and associated with red itchy eyes, or doctor diagnosis

\section{Sariachvili, 2010}

Nested Case-Control Study; Belgium (Influence of Perinatal Factors on the Occurrence of Asthma and Allergies Study)

Sample Size:

\section{Eczema cases: 252}

Controls: 305

Attrition: NA

Sample Size Calculation: NR

Sex: $50 \%$ Female

Race/Ethnicity: NR

\section{Intervention/Exposure:}

Egg (age)

Fish (age)

Milk products (age)

Grain products (age)

Other (fruit, vegetables, meat; age)

Assessment Methods: Parent questionnaire

\section{Outcomes:}

Eczema

Age: 0-4y

\section{Outcome assessors were not blinded}

Did not use valid/reliable measures (parent reported outcomes)

Did not adjust for key confounders (SES, race/ethnicity, birth size, gestational age, pets)

Did not report statistical results of analyses shown in figures
Confounders: The following confounders were taken into account: Education, Sex, Feeding practices, Birth size, Smoking, Atopy risk status, Birth order, maternal age

\section{Limitations:}

Cannot determine whether groups were similar at baseline on key characteristics

\section{Outcome assessors were not blinded}

Did not use valid/reliable measures (parent reported outcomes)

Did not adjust for key confounders (SES, race/ethnicity, gestational age, pets) 


\section{Subject Characteristics (Sample Size, Sex, \\ Race/Ethicity, Atopic Disease Risk Status; \\ Background Diet)}

Atopic Disease Risk Status: Parental allergy: 63\% cases,

$46 \%$ controls

Background Diet: BF: 46\% >4mo; FF: 70\%<4mo

CFB introduction: $45 \%<4 \mathrm{mo}$

Strassburger, 2010

Prospective Cohort Study; Brazil

Sample Size:

Baseline N: 397

Analytic N: 354

Attrition: $12 \%$

Sample Size Calculation: NR

Sex: $44 \%$ Female

Race/Ethnicity: NR

Atopic Disease Risk Status: Maternal asthma: 13\%;

Paternal asthma: $8 \%$

Background Diet: BF: $36 \%$ EBF for $>4 \mathrm{mo}, 12 \%$ EBF for

$>6 \mathrm{mo}$

Cow's milk: $40 \%<4 \mathrm{mo}$

Tromp, 2011 Intervention/Exposure:

Prospective Cohort Study; The Netherlands (Generation R Egg (age)

Study)

Sample Size:

Baseline N: 7893

Analytic N: 6905

Attrition: $13 \%$

\section{Independent Variable/Exposure, Outcomes}

\section{Assessment Methods:}

Eczema: Parent report of $1+$ episode Intervention/Exposure:

Other (fruit, salty pureed food; age)

Assessment Methods: Parent questionnaire

\section{Outcomes:}

Asthma

Age: 3-4y

Assessment Methods:

Asthma: Parent report
Did not report statistical results of analyses shown in figures, or adjusted results for timing of introduction of individual food items
Confounders: The following confounders were taken into account: SES, Sex, Birth size, Gestational age, RCT group

Limitations:

Cannot determine whether groups were similar at baseline on key characteristics

Outcome assessors were not blinded

Did not use valid/reliable measures (parent reported outcomes)

Cannot determine whether key confounders were adjusted for as the confounders included in adjusted analyses were not described
Grain products (age)

Peanuts, tree nuts, or seeds (age)

Soybeans (age)

Assessment Methods: Parent questionnaire; FFQ
Confounders: The following confounders were taken into account: Education, SES, Sex, Race/ethnicity, Feeding practices, Birth size, Gestational age, Smoking, Atopy risk status, Parity, child medication us, day care attendance

Limitations:

Cannot determine whether groups were similar at baseline on key characteristics 


\section{Subject Characteristics (Sample Size, Sex, \\ Race/Ethicity, Atopic Disease Risk Status; \\ Background Diet)}

\section{Sample Size Calculation: NR}

Sex: $49 \%$ Female

Race/Ethnicity: 63\% Dutch/ Western, 37\% non-Western

Atopic Disease Risk Status: Parental atopy: 47\%

Background Diet: BF: $89 \%$ initiated, 5\% EBF for $6 \mathrm{mo}$

\section{Turati, 2016}

Case-Control Study; Italy

Sample Size:

Atopic Dermatitis Cases: 451

Controls: 451

Attrition: NA

Sample Size Calculation: NR

Sex: $33 \%$ Female

Race/Ethnicity: NR

Atopic Disease Risk Status: NR

Background Diet: NR

Independent Variable/Exposure, Outcomes

\section{Confounders, Limitations}

\section{Outcome assessors were not blinded}

Outcomes:

Eczema

Age: 2y, 3y, 4y

Assessment Methods:

Eczema: Parent report

Intervention/Exposure:

Egg (age)

Fish (age)

Milk products (age)

Grain products (age)

Peanuts, tree nuts, or seeds (age)

Other (fruit, vegetables, meat; age)

Assessment Methods: Parent questionnaire

\section{Outcomes:}

Atopic dermatitis

Age: 3-24mo

Assessment Methods:

Atopic dermatitis: Physician diagnosis based on the following eight mandatory conditions: 'no previous diagnosis of AD', 'first symptoms occurring no longer than 5 months before AD diagnosis', 'symptoms occurring also in the last 4 weeks', 'the child is suffering from

itching', 'the child has eczematous lesions', 'age-specific affected areas', 'flexural
Did not use valid/reliable measures (parent reported outcomes)

Did not adjust for key confounders (pets)

Confounders: The following confounders were taken into account: Education, Sex, Race/ethnicity, Feeding practices, Gestational age, Smoking, Atopy risk status, Center, age, period of enrollment, maternal age, number of siblings, type of delivery, vitamin D supplementation

Limitations:

Inclusion and exclusion criteria not described, so cannot determine whether they were the same between cases/controls

Cannot determine whether groups were similar at baseline on key characteristics

Did not use valid/reliable measures (to assess dietary intake)

Did not adjust for key confounders (SES, birth size, smoking, pets)) 


\section{Subject Characteristics (Sample Size, Sex, Race/Ethicity, Atopic Disease Risk Status; \\ Background Diet)}

Independent Variable/Exposure, Outcomes

involvement', and 'the groin and armpits areas

are not affected, unless a diagnosis of inverted psoriasis'

\section{Venter, 2016}

Nested Case-Control Study; United Kingdom (Food

Allergy and Intolerance Research Cohort)

Sample Size:

Food Allergy Cases: 39

Controls: 78

Attrition: NA

Sample Size Calculation: N=96 cases and 192 controls

\section{Sex: 35\% Female}

Race/Ethnicity: Predominantly Caucasian

Atopic Disease Risk Status: Maternal allergy: 23\%;

Maternal hay fever: 35\%; Maternal eczema: 27\%;

Maternal food allergy: $20 \%$

Background Diet: BF: EBF for $1 \mathrm{mo}$

Age of CFB introduction: 112d

\section{Virtanen, 2010}

Prospective Cohort Study; Finland (Diabetes Prediction and Prevention Study)

Sample Size:

Baseline N: 1374

Analytic N: 1288

Attrition: $6 \%$

Sample Size Calculation: NR

Intervention/Exposure:

Egg (age)

Grain products (age)

Peanuts, tree nuts, or seeds (age)

Assessment Methods: Parent questionnaire

\section{Outcomes:}

Food allergy

Age: 1y

Assessment Methods:

Food allergy: SPT wheal $>3 \mathrm{~mm}$ and DBPCFC for milk, egg, wheat, cod, peanut, or sesame

\section{Intervention/Exposure:}

\section{Egg (age)}

Fish (age)

Milk products (age)

Grain products (age)

Other (fruit, vegetables, meat; age)

Assessment Methods: Parent questionnaire
Confounders: No confounders were accounted for

Limitations:

Cannot determine whether groups were similar at baseline on key characteristics

Cannot determine whether outcome assessors were blinded

Did not adjust for any key confounders (Education, SES, sex, race/ethnicity, feeding practices, birth size gestational age, smoking, atopy risk status, pets))
Confounders: The following confounders were taken into account: Sex, Feeding practices, Gestational age, Smoking, Atopy risk status, Maternal age, delivery mode, study area, siblings, pets

\section{Limitations:}

Cannot determine whether groups were similar at baseline on key characteristics 


\section{Subject Characteristics (Sample Size, Sex, Race/Ethicity, Atopic Disease Risk Status; \\ Background Diet)}

\section{Sex: $48 \%$ Female}

Race/Ethnicity: NR

Atopic Disease Risk Status: Parental asthma: 14\%; Parental allergic rhinitis: 60\%; Parental atopic eczema: $48 \%$

Background Diet: NR

\section{Independent Variable/Exposure, Outcomes}

Outcomes:

Asthma, Allergic rhinitis

Age: $5 y$

Assessment Methods:

Asthma: Parent report of doctor diagnosis with either wheezing or asthma medication use during the previous $12 \mathrm{mo}$

Allergic rhinitis: Parent report of sneezing, nasal congestion or rhinitis other than with respiratory infection with the symptoms of rhinitis eye itching and tearing

\section{Wei-Liang Tan, 2016}

Randomized Controlled Trial; Australia (Beating Egg Allergy Trial (BEAT))

Sample Size:

Egg Group Baseline N: 165

Rice Group Baseline N: 154

Egg Group Analytic N: 130

Rice Group Analytic N: 124

Attrition: $20 \%$

Sample Size Calculation: N=160/group had $80 \%$ power at a 2 -sided $5 \%$ a value to detect a difference in sensitization from $15 \%$ in the control group to $5 \%$ in the egg group

Sex: $48 \%$ Female

Race/Ethnicity: NR

Atopic Disease Risk Status: Familial atopy: 100\%; No response to a SPT to commercial egg white at $4 \mathrm{mo}$

Intervention/Exposure:

Egg (Egg vs. placebo from 4 to $8 \mathrm{mo}$ )

Assessment Methods: NA

Outcomes:

Food allergy (egg), Atopic eczema

Age: $4 \mathrm{mo}, 8 \mathrm{mo}, 12 \mathrm{mo}$

Assessment Methods:

Food allergy: OFC, reaction to egg at dietary introduction after $8 \mathrm{mo}$, and/or an egg-white SPT wheal $>3 \mathrm{~mm}$ at $12 \mathrm{mo}$; probable egg allergy defined as reaction to egg with IgEmediated symptoms within $1 \mathrm{hr}$ of ingestion and SPT response $>3 \mathrm{~mm}$ OR reaction to egg OFC OR SPT response $>5 \mathrm{~mm}$

Atopic eczema: Physical exam, with severity scores using SCORAD

\section{Confounders: N/A}

Limitations:

All subjects were high risk (familial atopy)
Cannot determine whether outcome assessors were

blinded

Did not use valid/reliable measures (to assess outcomes)

Did not adjust for any key confounders (Education, SES, race/ethnicity, birth size) 


\section{Subject Characteristics (Sample Size, Sex, \\ Race/Ethicity, Atopic Disease Risk Status; \\ Background Diet)}

Background Diet: BF: 45\% EBF at $4 \mathrm{mo}$

Independent Variable/Exposure, Outcomes

Confounders, Limitations

Yu, 2011

Case-Control Study; United States

Sample Size:

Allergic Cases: 67

Non-Allergic Controls: 191

Attrition: NA

Sample Size Calculation: NR

Sex: NR

Race/Ethnicity: NR

Atopic Disease Risk Status: NR

Background Diet: NR
Intervention/Exposure:

Egg (age)

Fish (age)

Milk products (age)

Grain products (age)

Peanuts, tree nuts, or seeds (age)

Assessment Methods: Parent questionnaire

\section{Outcomes:}

Food allergy

Age: 14-18y

Assessment Methods:

Food allergy: Student/parent report of food allergies and whether those allergies had been confirmed via IgE, SPT, and/or other

confirmation by a health care professional

\section{Zutavern, 2004}

Prospective Cohort Study; United Kingdom

Sample Size:

Baseline N: 642

Analytic N: 552

Attrition: $14 \%$

Sample Size Calculation: NR

Sex: $47 \%$ Female Intervention/Exposure:

\section{Egg (age)}

Fish (age)

Milk products (age)

Grain products (age)

Other (fruit, vegetables, meat; age)

Assessment Methods: Parent questionnaire

Confounders: No confounders were accounted for

Limitations:

Cannot determine whether groups were similar at baseline on key characteristics

Outcome assessors were not blinded

Did not use valid/reliable measures (to assess outcomes)

Did not adjust for any key confounders (Education, SES, sex, race/ethnicity, feeding practices, birth size, gestational age, smoking, atopy risk status, pets)

Small sample size, potentially underpowered

Confounders: The following confounders were taken

into account: Education, SES, Sex, Feeding practices, Birth size, Smoking, Atopy risk status, Number of rooms in house, birth order, number of siblings, maternal age

\section{Limitations:}

Cannot determine whether groups were similar at baseline on key characteristics

Outcome assessors were not blinded 


\section{Subject Characteristics (Sample Size, Sex, Race/Ethicity, Atopic Disease Risk Status; \\ Background Diet)}

Race/Ethnicity: NR

Atopic Disease Risk Status: Parental atopy: 63\%; Parental asthma: $25 \%$

Background Diet: BF: 63\% initiated

CFB introduction: $45 \%$ introduced to solids $>3 \mathrm{mo}$

Zutavern, 2006

Prospective Cohort Study; Germany (Lifestyle-Related Factors on the Immune System and the Development of Allergies in Childhood Study)

Sample Size:

Baseline N: 3097

Analytic N: 2612

Attrition: $16 \%$

Sample Size Calculation: NR

Sex: $48 \%$ Female

Race/Ethnicity: NR

Atopic Disease Risk Status: Parental atopy: 53\%

Background Diet: NR

\section{Independent Variable/Exposure, Outcomes}

\section{Outcomes:}

Eczema

Age: $5 y$

Assessment Methods:

Eczema: Parent report of doctor diagnosis

Intervention/Exposure:

Egg (age)

Fish (age)

Milk products (age)

Grain products (age)

Combined allergens (soybean, nut, cacao, and chocolate; age)

Other (fruit, vegetables, meat; age)

Assessment Methods: Parent questionnaire

Outcomes:

Atopic dermatitis

Age: $2 y$

Assessment Methods:

Atopic dermatitis: Parent report of doctor diagnosis in last 6mo ("doctor-diagnosed"); parent reported of itching eczema within the last $6 \mathrm{mo}$ that was either recurrent or lasted for 2wk and that affected the skin creases, face, neck, extremities, hands, feet, or trunk (not under diaper) ("active AD"); or parent report of doctor diagnosis or eczema related to food

\section{Did not use valid/reliable measures (to assess} outcomes)

Did not adjust for any key confounders (race/ethnicity, gestational age, pets))

Confounders: The following confounders were taken into account: Education, Sex, Feeding practices, Birth size, Smoking, Atopy risk status, Study center

\section{Limitations:}

Cannot determine whether groups were similar at baseline on key characteristics

Outcome assessors were not blinded

Did not use valid/reliable measures (to assess outcomes)

Did not adjust for any key confounders (SES, race/ethnicity, gestational age, pets)) 
Subject Characteristics (Sample Size, Sex, Race/Ethicity, Atopic Disease Risk Status;

Background Diet) 
Table 2. Summary of studies examining the relationship between diet diversity or dietary patterns during the complementary feeding period and food allergy, atopic dermatitis/eczema, asthma, and allergic rhinitis

\begin{tabular}{|c|c|c|}
\hline $\begin{array}{l}\text { Reference; Sample Size; Subject } \\
\text { Characteristics }\end{array}$ & Intervention/Exposure; Outcomes & Confounders; Limitations \\
\hline \multicolumn{3}{|l|}{ Diet Diversity } \\
\hline Fergusson, 1981 & Intervention/Exposure: & \multirow{2}{*}{$\begin{array}{l}\text { Confounders: The following confounders were taken } \\
\text { into account: Atopy risk status }\end{array}$} \\
\hline Prospective Cohort Study; New Zealand & Diet diversity at $4 \mathrm{mo}: 0,1-2,3-4,5+$ food groups & \\
\hline $\begin{array}{l}\text { (Christchurch Health and Development Study) } \\
\text { Sample Size: }\end{array}$ & $\begin{array}{l}\text { Food groups: cereals, vegetables, dairy products, meat, } \\
\text { fruits, egg or related products, other solid foods }\end{array}$ & Limitations: \\
\hline Baseline N: 1262 & Assessment Methods: Parent interview and food diary & $\begin{array}{l}\text { Cannot determine whether groups were similar at } \\
\text { baseline on key characteristics }\end{array}$ \\
\hline Millaty ut iv. 1100 & & \multirow{3}{*}{$\begin{array}{l}\text { Cannot determine recruitment methods, baseline } \\
\text { characteristics, confounding factors, or blinding } \\
\text { methods }\end{array}$} \\
\hline Attrition: $8 \%$ & Outcomes: & \\
\hline Sample Size Calculation: NR & Eczema & \\
\hline Sex: NR & Age: $2 y$ & Outcome assessors were not blinded \\
\hline Race/Ethnicity: NR & Assessment Methods: & \multirow{2}{*}{$\begin{array}{l}\text { Did not use valid/reliable measures to assess } \\
\text { outcomes (maternal report of eczema) }\end{array}$} \\
\hline $\begin{array}{l}\text { Atopic Disease Risk Status: } ~ 24 \% \text { had parental } \\
\text { atopy history }\end{array}$ & \multirow[t]{4}{*}{ Eczema: Maternal report; some with physician follow-up } & \\
\hline \multirow{3}{*}{$\begin{array}{l}\text { Background Diet: } ~ 19 \% \text { received "breast milk } \\
\text { only" at } 4 \text { months }(\mathrm{mo})\end{array}$} & & $\begin{array}{l}\text { Did not adjust for key confounders (education, SES, } \\
\text { sex, race/ethnicity, feeding practices, birth size, } \\
\text { gestational age, smoking, pets) }\end{array}$ \\
\hline & & $\begin{array}{l}\text { Examined diet diversity at a single time point early in } \\
\text { the CF period ( } 4 \mathrm{mo}) \text { when few subjects were } \\
\text { introduced to CFB }\end{array}$ \\
\hline & & $\begin{array}{l}\text { A small percentage of infants ( } 19 \% \text { ) were EBF at } \\
4 \text { mo, which may not be representative of current } \\
\text { infant feeding practices, and could have impact } \\
\text { eczema risk }\end{array}$ \\
\hline Fergusson, 1982 & Intervention/Exposure: & \multirow{3}{*}{$\begin{array}{l}\text { Confounders: The following confounders were taken } \\
\text { into account: Feeding practices }\end{array}$} \\
\hline Prospective Cohort Study; New Zealand & Diet diversity at $4 \mathrm{mo}: 0,1-3,4+$ food groups & \\
\hline $\begin{array}{l}\text { (Christchurch Health a } \\
\text { Sample Size: }\end{array}$ & $\begin{array}{l}\text { Food groups: cereals, vegetables, dairy products, meat } \\
\text { products, fruits, egg }\end{array}$ & \\
\hline
\end{tabular}




\begin{tabular}{|c|c|c|}
\hline $\begin{array}{l}\text { Reference; Sample Size; Subject } \\
\text { Characteristics }\end{array}$ & Intervention/Exposure; Outcomes & Confounders; Limitations \\
\hline $\begin{array}{l}\text { Baseline N: } 1265 \\
\text { Analytic N: } 1143\end{array}$ & Assessment Methods: Parent interview and food diary & $\begin{array}{l}\text { Cannot determine whether groups were similar at } \\
\text { baseline on key characteristics }\end{array}$ \\
\hline $\begin{array}{l}\text { Attrition: } 10 \% \\
\text { Sample Size Calculation: NR }\end{array}$ & $\begin{array}{l}\text { Outcomes: } \\
\text { Eczema }\end{array}$ & $\begin{array}{l}\text { Cannot determine recruitment methods, baseline } \\
\text { characteristics, confounding factors, or blinding } \\
\text { methods }\end{array}$ \\
\hline Sex: NR & Age: $3 y$ & Outcome assessors were not blinded \\
\hline $\begin{array}{l}\text { Race/Ethnicity: NR } \\
\text { Atopic Disease Risk Status: 24\% had parental } \\
\text { atopy history } \\
\text { Background Diet: NR }\end{array}$ & $\begin{array}{l}\text { Eczema: Cumulative rates based on maternal report; } \\
\text { some with physician follow-up }\end{array}$ & $\begin{array}{l}\text { Did not use valid/reliable measures to assess } \\
\text { outcomes (maternal report of eczema) } \\
\text { Did not adjust for key confounders (education, SES, } \\
\text { sex, race/ethnicity, birth size, gestational age, } \\
\text { smoking, atopy risk status, pets) }\end{array}$ \\
\hline $\begin{array}{l}\text { Fergusson, } 1990 \\
\text { Prospective Cohort Study; New Zealand } \\
\text { (Christchurch Health and Development Study) } \\
\text { Sample Size: }\end{array}$ & $\begin{array}{l}\text { Intervention/Exposure: } \\
\text { Diet diversity at } 4 \mathrm{mo}: 0,1-3,4+\text { food groups } \\
\text { Food groups: cow's milk, cereals, vegetables, dairy } \\
\text { products, meat, fruit, egg/related products, other foods }\end{array}$ & $\begin{array}{l}\text { Confounders: The following confounders were taken } \\
\text { into account: Education, SES, Sex, Race/ethnicity, } \\
\text { Feeding practicies, Atopy risk status, Birth order }\end{array}$ \\
\hline $\begin{array}{l}\text { Baseline N: } 1265 \\
\text { Analytic N: } 1067 \\
\text { Attrition: } 15.6 \%\end{array}$ & Assessment Methods: Parent interview and food diary & $\begin{array}{l}\text { Cannot determine recruitment methods, baseline } \\
\text { characteristics, confounding factors, or blinding } \\
\text { methods }\end{array}$ \\
\hline Sample Size Calculation: NR & Eczema & Outcome assessors were not blinded \\
\hline $\begin{array}{l}\text { Sex: NR } \\
\text { Race/Ethnicity: NR }\end{array}$ & $\begin{array}{l}\text { Age: } 10 y \\
\text { Assessment Meth }\end{array}$ & $\begin{array}{l}\text { Did not use valid/reliable measures to assess } \\
\text { outcomes (maternal report of eczema) }\end{array}$ \\
\hline $\begin{array}{l}\text { Atopic Disease Risk Status: } 44 \% \text { of eczema } \\
\text { cases had } 1 \text { or both parents with eczema; } 57 \% \\
\text { of eczema cases parents had history of asthma } \\
\text { or allergic rhinitis } \\
\text { Backaround Diet: NR }\end{array}$ & \multirow[t]{2}{*}{$\begin{array}{l}\text { Eczema: Chronic, recurrent based on maternal report; } \\
\text { some with physician follow-up }\end{array}$} & \multirow[t]{2}{*}{$\begin{array}{l}\text { Examined diet diversity at a single time point early in } \\
\text { the CF period ( } 4 \mathrm{mo}) \text { when few subjects were } \\
\text { introduced to CFB }\end{array}$} \\
\hline Background Diet: NR & & \\
\hline
\end{tabular}


The period of follow-up (10y) may have missed the timeframe during which outcomes were expected to be seen

\section{Fergusson, 1994 \\ Prospective Cohort Study; New Zealand} (Christchurch Health and Development Study)

Sample Size:

Baseline N: 1262

Analytic N: 1141

Attrition: $9.6 \%$

Sample Size Calculation: NR

Sex: NR

Race/Ethnicity: NR

Atopic Disease Risk Status: Of those who had severe eczema, $65 \%$ had a history of asthma or allergic rhinitis; $22 \%$ food allergy

\section{Background Diet: NR}

\section{Intervention/Exposure:}

Diet diversity at 4mo: 0, 1-3, 4+ food groups

Food groups: cows milk, cereals, eggs, vegetables, fruit, meat, dairy products, other foods

Assessment Methods: Parent interview and food diary

Outcomes:

\section{Eczema}

Age: $10 y$

Assessment Methods:

Eczema: Maternal report; some with physician follow-up

Confounders: The following confounders were taken into account: Education, SES, Sex, Feeding pracrtices, Atopy risk status

\section{Limitations:}

Cannot determine whether groups were similar at baseline on key characteristics

Cannot determine recruitment methods, baseline characteristics, confounding factors, or blinding methods

\section{Outcome assessors were not blinded}

Did not use valid/reliable measures to assess outcomes (maternal report of eczema)

Did not adjust for key confounders (race/ethnicity, birth size, gestational age, smoking, pets)

Examined diet diversity at a single time point early in the CF period (4mo) when few subjects were introduced to CFB

The period of follow-up (10y) may have missed the timeframe during which outcomes were expected to be seen

Prospective Cohort Study; Germany (German Infant Nutritional Intervention Program)

\section{Sample Size:}

Baseline N: 5991 ( $\mathrm{N}=2252$ in intervention, $\mathrm{N}=3739$ in non-intervention groups)

Diet diversity at 4mo: 0, 1-2, 3-8 food groups

Diet diversity at $6 \mathrm{mo:}$ 0, 1-2, 3-4, 5-8 food groups

Food groups: vegetables, cereal, fruit, meat, dairy, egg, fish, other (nuts/cocoa/chocolate/ soybean)
Confounders: The following confounders were taken into account: Feeding practices, Atopy risk status, Sex, Study region, Siblings, Education, Smoking, Birth weight, Pets

Limitations: 


\section{Reference; Sample Size; Subject Characteristics}

Analytic N: 4753

Attrition: 21\%

Sample Size Calculation: NR

Sex: NR

Race/Ethnicity: NR

Atopic Disease Risk Status: Familial history of eczema: intervention 39.9\%, non-intervention $9.8 \% ; 100 \%$ of subjects in the intervention group had family history of atopic disease

Background Diet: $~ 50 \%$ EBF for $4 \mathrm{mo}$

\section{Nwaru, 2014}

Prospective Cohort Study; Finland (Finnish Type 1 Diabetes Prediction and Prevention Study)

Sample Size:

\section{Baseline N: 4,075}

Analytic N: 3,142

Attrition: $22.9 \%$

Sample Size Calculation: NR

Sex: $48 \%$ Female

Race/Ethnicity: NR

Atopic Disease Risk Status: $16 \%$ with parental asthma; $62 \%$ with parental allergic rhinitis

Background Diet: NR
Intervention/Exposure; Outcomes

Assessment Methods: Parent questionnaire

Outcomes:

Eczema

Age: 4y

Assessment Methods:

Eczema: Parent report of doctor diagnosis or recurrent symptoms lasting for $6 \mathrm{mo}$ up to age $1 \mathrm{y}$ and for $2 \mathrm{wk}$ at age 2-4y

\section{Confounders; Limitations}

Cannot determine whether groups were similar at baseline on key characteristics

Outcome assessors (parent report) were not blinded

Did not use valid/reliable measures to assess outcomes (parent report of physician diagnosed eczema)

Did not adjust for key confounders (SES, race/ethnicity, gestational age, pets)

Multi-component intervention may have confounded outcome (randomization to four different formulas+ recommendations)

Sample size calculations not reported

Intervention/Exposure:

Diet diversity at 3mo: 0, 1-2, 2+ food groups

Diet diversity at 4mo: 0, 1-2, 3-4, 4+ food groups

Diet diversity at 6mo: 0-4, 5-6, 7-8, 8+ food groups

Diet diversity at $12 \mathrm{mo}: 0-7,8-9,10-11,11+$ food groups

Food groups: cow's milk and infant formula; potatoes; carrots; turnip; fruits and berries (as a combined variable); cereals (rye, wheat, oats, and barley as a combined variable); other cereals (maize, rice, millet, and buckwheat as a combined variable), meat; fish; egg; cabbage; spinach; and lettuce

Assessment Methods: Parent questionnaire

\section{Outcomes:}

Asthma, atopic asthma, nonatopic asthma (asthma not asssociated with any other atopic disease), allergic rhinitis, atopic eczema
Confounders: The following confounders were taken into account: Education, Sex, Birth size, Gestational age, Smoking, Atopy risk status, Number of siblings, Delivery hospital, Season of birth, Maternal age, Pets at home, Delivery mode

Cannot determine whether groups were similar at

Did not use valid/reliable measures for all outcomes

Did not adjust for key confounders (SES, race/ethnicity, feeding practices)

Multiple comparisons could increase risk of type 1 error

Cannot determine whether the associations reported are due to any diversity of food intake, or if certain food groups contributed more than others

\section{Limitations:} baseline on key characteristics 


\section{Roduit, 2012}

Prospective Cohort Study; Austria, Finland, France, Germany, Switzerland (Protection Against Allergy-Study in Rural Environments Study)

Sample Size:

Baseline N: 1,133

Analytic N: 1,041

Attrition: $8.1 \%$

Sample Size Calculation: NR

Sex: NR

Race/Ethnicity: NR

Atopic Disease Risk Status: $53.6 \%$ had at least 1 allergic parent

Background Diet: At $2 \mathrm{mo}, 66 \%$ were EBF; $46.4 \%$ BF for $>6 \mathrm{mo} ; 1.7 \%$ received no CFB by $12 \mathrm{mo}$

\section{Age: 5y}

Assessment Methods:

Asthma: Parent report of doctor-diagnosed asthma plus either any wheezing symptom or use of asthma medication during last $12 \mathrm{mo}$ (validated)

Allergic rhinitis: Parent report of sneezing, nasal congestion, or rhinitis other than with respiratory tract infections accompanied by itching of the eye and tearing during last $12 \mathrm{mo}$

Atopic eczema: Parent report of ever diagnosed by doctor

Cannot rule out potential for reverse causality, where children with early symptoms (e.g. wheezing) may have received restricted diets

Confounders: The following confounders were taken into account: Education, Feeding practices, Smoking, Atopy risk status, Study center, Farming

Diet diversity from 3-12mo: 0-3, 4-5, 6 food groups

Food groups: vegetables or fruits, cereals, bread, meat, cake, and yogurt (food items introduced in the diet of $80 \%$ of the children by $12 \mathrm{mo}$ )

Assessment Methods: Food diary

\section{Outcomes:}

Atopic dermatitis

Age: 1y, 4y

Assessment Methods:

Atopic dermatitis: Parent report that the child had atopic dermatitis diagnosed by a doctor at least once between $12 \mathrm{mo}$ and $4 \mathrm{y}$, positive SCORAD scores $(>0)$ assessed at the age of $1 \mathrm{y}$ during medical examination, or both

\section{Limitations:}

Cannot determine whether groups were similar at baseline on key characteristics

Outcome assessors were not blinded

Did not use valid/reliable measures to assess outcomes (parent reported atopic dermatitis)

Did not adjust for key confounders (SES, sex, race/ethnicity, birth size, gestational age, pets)

Did not report statistical results of analyses shown in figures 
Among children with allergic parents, allergenic

food items, such as dairy products, egg, nut,

and soy, were introduced later

\section{Roduit, 2014 \\ Prospective Cohort Study; Austria, Finland,}

France, Germany, Switzerland (Protection

Against Allergy-Study in Rural Environments Study)

Sample Size:

Baseline N: 1,133

Analytic N: 856

Attrition: $24.4 \%$

Sample Size Calculation: NR

Sex: $49.5 \%$ Female

Race/Ethnicity: NR

Atopic Disease Risk Status: $53.6 \%$ had at least 1 allergic parent

Background Diet: $9.4 \%$ never BF, $26.4 \%$ BF $>10 \mathrm{mo}$

Among children with allergic parents, allergenic food items, such as dairy products, egg, nut, and soy, were introduced later

\section{Intervention/Exposure:}

Diet diversity from 3-12mo: 0-3, 4-5, 6 food groups

Food groups: vegetables or fruits, cereals, bread, meat, cake, and yogurt (food items introduced in the diet of $80 \%$ of the children by $12 \mathrm{mo}$ )

Assessment Methods: Food diary

\section{Outcomes:}

Food allergy, asthma (any asthma, atopic asthma, nonatopic asthma), allergic rhinitis

Age: $6 y$

Assessment Methods:

Food allergy: Parents reported up to age 6y that the child had at least once been given a diagnosis of food allergy by a doctor

Asthma: Parents reported at least once that the child had either doctor-diagnosed asthma or at least 2 doctor-

diagnosed episodes of obstructive bronchitis in the last 12 mo

Allergic rhinitis: Parent reported presence of symptoms (itchy, runny, or blocked nose without a cold and associated with red itchy eyes) or doctor-diagnosed allergic rhinitis

\section{Schoetzau, 2002}

Prospective Cohort Study; Germany (German Infant Nutritional Intervention Program)
Diet diversity at 24wk: Continuous, total number of food groups consumed

\section{Intervention/Exposure:}

Confounders: The following confounders were taken into account: Education, Sex, Feeding practices, Smoking, Atopy risk status, Study center, Farmer, Number of siblings

\section{Limitations:}

Cannot determine whether groups were similar at baseline on key characteristics

Outcome assessors were not blinded

Did not use valid/reliable measures (parent reported outcomes)

Did not adjust for key confounders (SES, race/ethnicity, birth size, gestational age, pets)

Did not report statistical results of analyses shown in figures

Confounders: The following confounders were taken into account: Education, Sex, Race/ethnicity, Feeding practices, Gestational age, Smoking, Atopy risk status, Pets 


\section{Reference; Sample Size; Subject}

Characteristics

Sample Size:

Baseline N: 2,252

Analytic N: 1,121

Attrition: $C D$

Sample Size Calculation: NR

Sex: $49 \%$ Female

Race/Ethnicity: NR

Atopic Disease Risk Status: $42 \%$ with $1+$ core family members with atopic dermatitis

Background Diet: $77 \%$ BF, 23\% fed cow's milk (excluded FF infants from the original studt)

\section{Turati, 2016}

Case-control study; Italy

Sample Size:

Cases: 451

\section{Controls: 451}

\section{Attrition: NA}

Sample Size Calculation: NR

\section{Sex: 33\% Female}

Race/Ethnicity: NR

Atopic Disease Risk Status: NR

Background Diet: All infants were weaned by $6 \mathrm{mo}$

\section{Intervention/Exposure; Outcomes}

Food groups: dairy products, egg, cereals, legumes,

vegetables, fruits, nuts, meat products, fish, and other foods

Assessment Methods: Food diary

Outcomes:

Atopic dermatitis

Age: 1y

Assessment Methods:

Atopic dermatitis: Physician diagnosis defined by a combination of diagnostic criteria, including typical morphology and distribution of skin lesions (face, neck and scalp, flexural folds, hands and extensor sides of extremities); pruritus (signs of scratching); and a tendency towards chronicity (duration of least $14 \mathrm{~d}$ and/or relapsing)

Intervention/Exposure:

Diet diversity at 4mo: 0, 1-2, 3-22 food groups

Diet diversity at 5mo: 0, 1-7, 8-22 food groups

Food groups: vegetables, legumes, or roots (potatoes, carrots, tomatoes, and beans), fruit (apples, pears, peaches, apricots, plums, citrus fruits, red fruits), cereals (maize/tapioca, rice, pasta, and gluten-free pasta), meat (poultry, pork, and beef), dairy products (cheese, and other dairies), fish, eggs, and nuts/cacao/chocolate

Assessment Methods: Parent questionnaire

\section{Outcomes:}

Atopic dermatitis

Age: $3-24 \mathrm{mo}$

\section{Confounders; Limitations}

\section{Limitations:}

Cannot determine whether groups were similar at baseline on key characteristics

Did not adjust for key confounders (SES, birth size)

Cannot rule out reverse causality

Confounders: The following confounders were taken into account: Education, Sex, Race/ethnicity, Feeding practices, Gestational age, Atopy risk status, Center, Age, Nutrient intake, Period of enrollment, Maternal age, Number of siblings, Type of delivery

\section{Limitations:}

Inclusion and exclusion criteria not described, so cannot determine whether they were the same between cases/controls

Cannot determine whether groups were similar at baseline on key characteristics

Did not use valid/reliable measures to assess dietary intake 


\section{Assessment Methods:}

Atopic dermatitis: Physician diagnosed based on the following eight mandatory conditions: 'no previous diagnosis of AD', 'first symptoms occurring no longer than 5 months before AD diagnosis', 'symptoms occurring also in the last 4 weeks', 'the child is suffering from itching', 'the child has eczematous lesions', 'age-specific affected areas', 'flexural involvement', and 'the groin and armpits areas are not affected, unless a diagnosis of inverted psoriasis'

\section{Zutavern, 2006}

Prospective Cohort Study; Germany (LifestyleRelated Factors on the Immune System and the Development of Allergies in Childhood)

\section{Sample Size:}

Baseline N: 3097

Analytic N: 2612

Attrition: $15.7 \%$

\section{Sample Size Calculation: NR}

Sex: $48 \%$ Female

\section{Race/Ethnicity: NR}

Atopic Disease Risk Status: 53\% with parental history of atopy

Background Diet: 59\% EBF at 4mo, 35.7\% mixed fed, $5.2 \%$ formula fed

\section{Intervention/Exposure:}

Diet diversity at $4 \mathrm{mo}:$ 0, 1-2, 3-8 food groups

Diet diversity at 6mo: 0, 1-2, 3-4, 5-8 food groups

Food groups: vegetables, cereal, fruit, meat, dairy products (milk), egg, fish, others (soybean, nuts, cacao, chocolate) (from 48 single food items)

Assessment Methods: Parent questionnaire

\section{Outcomes:}

Atopic dermatitis

Age: $2 y$

Assessment Methods:

Doctor-diagnosed atopic dermatitis: when parents reported a physician's diagnosis of atopic dermatitis in the last $6 \mathrm{mo}$

Symptomatic atopic dermatitis: when parents reported an itching eczema within the last 6 mo that was either recurrent or lasted for $2 \mathrm{wk}$ and that affected the skin creases, face, neck, extremities, hands, feet, or trunk (not underneath the diaper)
Did not adjust for key confounders (SES, birth size, smoking, pets)

Confounders: The following confounders were taken into account: Education, Sex, Feeding practices, Birth size, Smoking, Atopic risk status, Study center,

Number of siblings

Limitations:

Cannot determine whether groups were similar at baseline on some key characteristics

Outcome assessors were not blinded

Did not use valid/reliable measures (to assess outcomes)

Did not adjust for any key confounders (SES, race/ethnicity, gestational age, pets)

The period of follow-up (6y) may have missed the timeframe during which outcomes were expected to be seen 
Early skin or allergic symptoms: when parents gave an affirmative response to the question, "Has a doctor diagnosed your child with 1 of the following conditions within the first $6 \mathrm{mo}$ of life: atopic dermatitis; allergic or atopic eczema; food allergy, hives, urticaria, or allergic edema; milk crust or seborrheic eczema; eczema without further specification?" or when parents reported an increase of eczema as a result of food intolerance within the first $6 \mathrm{mo}$ of their child's life

\section{Zutavern, 2008}

Prospective Cohort Study; Germany (LifestyleRelated Factors on the Immune System and the Development of Allergies in Childhood)

\section{Sample Size:}

Baseline N: 3097

Analytic N: 2073

Attrition: $33.1 \%$

Sample Size Calculation: NR

Sex: $49.2 \%$ Female

Race/Ethnicity: NR

Atopic Disease Risk Status: 53\% with parental history of atopy

\section{Background Diet:}

$59 \% \mathrm{EBF}$ at $4 \mathrm{mo}, 35.7 \%$ mixed fed, $5.2 \%$ formula fed

Intervention/Exposure:

Diet diversity at $4 \mathrm{mo}:$ 0, 1-2, 3-8 food groups

Food groups: vegetables, cereal, fruit, meat, dairy products (milk), egg, fish, others (egg, soybean, nuts, cacao, chocolate) (from 48 single food items)

Assessment Methods: Parent questionnaire

Outcomes:

Atopic dermatitis, asthma, allergic rhinitis

Age: $6 y$

Assessment Methods:

Atopic dermatitis: Parent report of doctor diagnosis, or report of itching eczema within the last 6 mo that was recurrent or lasted for $2 \mathrm{wk}$ and that affected the skin creases, face, neck, extremities, hands, feet, or trunk (not underneath the diaper)

Asthma: Parent report of doctor diagnosis of asthma or reported the child's wheezing or intake of asthma medication

Allergic rhinitis: Parent report of doctor diagnosis of allergic rhinitis, or reported sneezing or a blocked or running nose of the child without having a cold
Confounders: The following confounders were taken

into account: Education, Sex, Birth weight, Feeding practicies, Smoking, Atopic risk status, Study center, Number of older siblings, Maternal age

\section{Limitations:}

Cannot determine whether groups were similar at baseline on key characteristics

Outcome assessors were not blinded

Did not use valid/reliable measures (to assess outcomes)

Did not adjust for any key confounders (SES race/ethnicity, gestational age, pets) 


\section{Characteristics}

\section{Dietary Patterns}

Nested Case-Control Study; United Kingdom (Prevalence of Infant Food Allergy EuroPrevall)

\section{Sample Size:}

Food Allergy Cases: 41

Controls: 82

\section{Attrition: NA}

Sample Size Calculation: NR

Sex: $46 \%$ Female

Race/Ethnicity: 95\% Caucasian

Atopic Disease Risk Status: Maternal atopy: $88 \%$ cases, $63 \%$ controls; Paternal atopy: $58 \%$ cases, $61 \%$ controls; Maternal asthma: $27 \%$ cases, $13 \%$ controls; Maternal allergy: $54 \%$ cases, $38 \%$ controls

\section{Background Diet: NR}

Grimshaw, 2014

\section{Intervention/Exposure:}

Adherence to dietary patterns from 0-12 mo, identified using principal component analysis:

'Infant guidelines' pattern: high scores for 'healthy' foods [commercial baby food, toddler snacks, carrots, potatoes, bananas, lentils, broccoli) and low scores for adult foods (potato products, ready meals, sauces)

'Finger food' pattern: high scores for healthy finger foods and low scores for unhealthy finger foods and pureed baby food

'Adult food pattern': high scores for adult foods and low scores for 'healthy' foods

Assessment Methods: Food diary

Outcomes:

Food allergy

Age: $2 y$

Assessment Methods:

Food allergy: Parent report with clinical history, IgE $\geq 0.35$ and/or SPT wheal $\geq 3 \mathrm{~mm}$; exclusion diet with improved symptoms; and DBPCFC (including delayed reactions up to $48 \mathrm{hr}$ after the challenge)

\section{Grimshaw, 2016}

Nested Case-Control Study; United Kingdom (Prevalence of Infant Food Allergy EuroPrevall) Sample Size:

Food Allergy Cases: 41

\section{Intervention/Exposure:}

Adherence to a dietary pattern from $0-12 \mathrm{mo}$, identified using principal component analysis:

'Healthy dietary' pattern: high scores for 'healthy' foods [commercial baby food, toddler snacks, carrots, potatoes,
Confounders: The following confounders were taken into account: Education, Sex, Feeding practices, Smoking, Atopy risk status, Pet ownership, Siblings, Maternal Age

Limitations:

Cannot determine whether outcome assessors were blinded

Cannot determine whether participants were similar at baseline on key characteristics

Did not adjust for key confounders (SES, race/ethnicity, birth size, gestational age)

Potentially underpowered due to small number of cases included

Confounders: The following confounders were taken into account: Gestational age, Atopy risk status, Age, Dog at home, Received skin creams, powders, lotions, No nut/peanut intake during pregnancy, Reduced soy intake during BF 


\begin{tabular}{|c|c|c|}
\hline $\begin{array}{l}\text { Reference; Sample Size; Subject } \\
\text { Characteristics }\end{array}$ & Intervention/Exposure; Outcomes & Confounders; Limitations \\
\hline $\begin{array}{l}\text { Controls: } 82 \\
\text { Attrition: NA } \\
\text { Sample Size Calculation: NR } \\
\text { Sex: } 46 \% \text { Female } \\
\text { Race/Ethnicity: } 95.8 \% \text { Caucasian } \\
\text { Atopic Disease Risk Status: Maternal atopy: } \\
88 \% \text { cases, } 63 \% \text { controls; Paternal atopy: } 58 \% \\
\text { cases, } 61 \% \text { controls; Maternal asthma: } 27 \% \\
\text { cases, } 13 \% \text { controls; Maternal allergy: } 54 \% \\
\text { cases, } 38 \% \text { controls } \\
\text { Background Diet: NR }\end{array}$ & $\begin{array}{l}\text { Outcomes: } \\
\text { Food allergy } \\
\text { Age: } 2 y \\
\text { Assessment Methods: } \\
\text { Food allergy: Parent report with clinical history, lgE } \geq 0.35 \\
\text { and/or SPT wheal } \geq 3 \mathrm{~mm} \text {; exclusion diet with improved } \\
\text { symptoms; and DBPCFC (including delayed reactions up } \\
\text { to } 48 \mathrm{hr} \text { after the challenge) }\end{array}$ & $\begin{array}{l}\text { Limitations: } \\
\text { Cannot determine whether participants were similar } \\
\text { at baseline on key characteristics } \\
\text { Did not account for high rate of attrition } \\
\text { Did not adjust for key confounders (SES, feeding } \\
\text { practices, smoking, pets) } \\
\text { Potentially underpowered due to small number of } \\
\text { cases included }\end{array}$ \\
\hline
\end{tabular}


Table 3. Results of studies that examined the relationship between peanut, tree nut, and/or seed product consumption during the complementary feeding period and risk of atopic disease.

\begin{tabular}{|c|c|c|}
\hline $\begin{array}{l}\text { Reference; Study design (Study or cohort } \\
\text { name); Country; Sample size }\end{array}$ & Intervention or exposure & Results \\
\hline \multirow[t]{3}{*}{$\begin{array}{l}\text { Du Toit, } 2015 \text { (1); Randomized Controlled } \\
\text { Trial (Learning Early About Peanut Allergy } \\
\text { Trial (LEAP)); UK; Peanut avoidance group: } \\
\text { 295, Peanut consumption group: } 294\end{array}$} & $\begin{array}{l}\text { Peanut consumption group: Peanuts (at least } 6 \\
\text { g/wk via } 3+\text { meals) from } 4 \text { to } 60 \mathrm{mo}\end{array}$ & $\begin{array}{l}\text { Peanut allergy at } 5 y \text {, subjects with a negative } \\
\text { baseline skin prick test: } 1.9 \% \text { v. } 13.7 \% \text {; } \\
\mathrm{P}<0.001 \text { ); absolute risk: } 11.8 \% \text { points, } 95 \% \\
\mathrm{Cl}: 3.4,20.3 \text {, or } 86 \% \text { risk reduction }\end{array}$ \\
\hline & $\begin{array}{l}\text { Peanut avoidance group: Avoided peanuts until } \\
60 \mathrm{mo}\end{array}$ & $\begin{array}{l}\text { Peanut allergy at } 5 y \text {, subjects with a positive } \\
\text { baseline skin prick test: } 10.6 \% \text { vs. } 35.3 \% \text {; } \\
P=0.004 \text {; absolute risk: } 24.7 \% \text { points, } 95 \% \mathrm{Cl} \text { : } \\
4.9,43.3 \text {, or } 70 \% \text { risk reduction }\end{array}$ \\
\hline & & Asthma at 5y: No significant group differences \\
\hline \multirow{2}{*}{$\begin{array}{l}\text { Filipiak, } 2007 \text { (3); Prospective Cohort Study } \\
\text { (German Infant Nutritional Intervention } \\
\text { Program); Germany; N: } 4753\end{array}$} & Age of nuts/cocoa introduction: $>6$ vs. $<6 \mathrm{mo}$ & $\begin{array}{l}\text { Atopic dermatitis (doctor-diagnosed) at 4y: } \\
\text { OR }=0.71 .95 \% \mathrm{Cl}: 0.50,1.00\end{array}$ \\
\hline & & $\begin{array}{l}\text { Atopic dermatitis (sympomatic) at } 4 \mathrm{y} \text { : } \\
\mathrm{OR}=0.70,95 \% \mathrm{Cl}: 0.50,0.99\end{array}$ \\
\hline $\begin{array}{l}\text { Greenhawt, } 2016 \text { (4); Prospective Cohort } \\
\text { Study (using RCT data from the LEAP trial); } \\
\text { UK; Peanut Avoidance Group = 295, Peanut } \\
\text { Consumption Group }=294\end{array}$ & Age of peanut introduction: $6-11$ vs $4-6 \mathrm{mo}$ & $\begin{array}{l}\text { Peanut allergy at 5y: Reduced risk for 6-11 vs. } \\
4-6 \text { mo, data not provided }\end{array}$ \\
\hline $\begin{array}{l}\text { Grimshaw, } 2013 \text { (10); Nested Case-Control } \\
\text { Study (Prevalence of Infant Food Allergy } \\
\text { EuroPrevall); UK; Food Allergy Cases: 41, }\end{array}$ & $\begin{array}{l}\text { Age of peanut, tree nut, and/or sesame } \\
\text { (combined) introduction: Food allergy cases vs. } \\
\text { controls consuming by } 52 \mathrm{wk}\end{array}$ & Food allergy at 2y: No significant associations \\
\hline
\end{tabular}

Age of peanut introduction: Number of consuming infants by $52 \mathrm{wk}$

Age of sesame introduction: Number of consuming infants by $52 \mathrm{wk}$
Food allergy at $2 \mathrm{y}: 12.2 \%$ vs. $7.3 \% ; P=0.055$

Food allergy at $2 \mathrm{y}: 12.2 \%$ vs. $9.8 \% ; \mathrm{P}=0.051$
Grimshaw, 2015 (9); Nested Case-Control

Study (Prevalence of Infant Food Allergy
Age of peanut introduction: Median in weeks, food allergy cases vs. controls
Food allergy (IgE and non-lgE-mediated)

cases at 2y: No significant associations 


\begin{tabular}{ll}
\hline \hline $\begin{array}{l}\text { Reference; Study design (Study or cohort } \\
\text { name); Country; Sample size }\end{array}$ & Intervention or exposure \\
\hline $\begin{array}{l}\text { EuroPrevall); UK; Food Allergy Cases: } 41 \\
\text { (21 lgE-mediated), Controls: } 82\end{array}$ & \\
\hline $\begin{array}{l}\text { Kumar, 2010 (11); Nested Case-Control } \\
\text { Study; US; Food Allergy Cases: 411, }\end{array}$ & Age of egg, peanut, tree nut, shellfish, fish, and \\
Asymptomatic Sensitized Cases: 171, & \\
Controls: 378 & \\
& \\
& \\
& \\
& \\
& Age of egg, peanut, tree nut, shellfish, fish, and \\
& sesame (combined) introduction: $>1$ vs. $<1 \mathrm{y}$
\end{tabular}

Results

sesame (combined) introduction: $>1$ vs. <1y

Food allergy at $\sim 6 y$, children without atopic dermatitis: No significant associations

Food allergy at $\sim 6 y$, children with atopic

dermatitis: No significant associations

Food allergy at $\sim 6 y$ : No significant associations

Food allergy at $\sim 6 y$, children without atopic dermatitis: $\mathrm{OR}=0.5,95 \% \mathrm{Cl}: 0.3,0.95 ; \mathrm{P}<0.05$

Food allergy at $\sim 6 y$, children with atopic dermatitis: No significant associations

Perkin, 2016 (2); Randomized Controlled Trial (Enquiring about Tolerance Trial (EAT); UK; Standard Introduction Group: 595, Early Introduction Group: 567
Early introduction group: Introduction to allergenic foods at 3mo (cow's milk (yogurt) first, then (in random order) peanut, cooked (boiled) hen's egg, sesame, and whitefish, and wheat last)

Standard introduction group: Introduction to allergenic foods at $6 \mathrm{mo}$ according to parental discretion
Food allergy from 1-3y, intention to treat analyses: No significant group differences based on intention to treat analyses

Food allergy from 1-3y, per protocol analyses: $2.4 \%$ vs. $6.4 \% ; P=0.03$

Peanut allergy from 1-3y, per protocol analyses: $0 \%$ vs. $2.5 \% ; P=0.003$

Sesame allergy from 1-3y, per protocol analyses: No significant group differences

Amount of peanut consumed: $2 \mathrm{~g} / \mathrm{wk}$ vs. $<2 \mathrm{~g} / \mathrm{wk}$ from 3 to $6 \mathrm{mo}$ (early introduction group Peanut allergy from 1-3y: Reduced risk with only) 


\begin{tabular}{|c|c|c|}
\hline $\begin{array}{l}\text { Reference; Study design (Study or cohort } \\
\text { name); Country; Sample size }\end{array}$ & Intervention or exposure & Results \\
\hline \multirow{3}{*}{$\begin{array}{l}\text { Roduit, } 2012 \text { (6); Prospective Cohort Study } \\
\text { (Protection Against Allergy in Rural } \\
\text { Environments Study); Austria, Finland, } \\
\text { France, Germany, Switzerland; N: } 1041\end{array}$} & Age of nut introduction: $3-12$ vs. $>12 \mathrm{mo}$ & $\begin{array}{l}\text { Atopic dermatitis at } 1 \mathrm{y}: \mathrm{OR}=0.54,95 \% \mathrm{Cl} \text { : } \\
0.33,0.90 ; P<0.05\end{array}$ \\
\hline & & $\begin{array}{l}\text { Atopic dermatitis (asymptomatic) at } 1 \mathrm{y} \text { : } \\
\mathrm{OR}=0.51,95 \% \mathrm{Cl}: 0.26,0.99 ; \mathrm{P}<0.05\end{array}$ \\
\hline & & $\begin{array}{l}\text { Atopic Dermatitis at } 4 y \text { : No significant } \\
\text { associations }\end{array}$ \\
\hline \multirow{2}{*}{$\begin{array}{l}\text { Roduit, } 2014 \text { (5); Prospective Cohort Study } \\
\text { (Protection Against Allergy in Rural } \\
\text { Environments Study); Austria, Finland, } \\
\text { France, Germany, Switzerland: N: } 856\end{array}$} & Age of nuts introduction: $<12$ mo vs. Never & Food allergy at 6y: No significant associations \\
\hline & & Ashma at 6y: No significant associations \\
\hline $\begin{array}{l}\text { Tromp, } 2011 \text { (7); Prospective Cohort Study } \\
\text { (Generation R Study); The Netherlands; N: } \\
6905\end{array}$ & $\begin{array}{l}\text { Age of peanut or tree nut introduction: Not } \\
\text { defined }\end{array}$ & $\begin{array}{l}\text { Atopic dermatitis at } 2,3 \text {, and } 4 \text { : No significant } \\
\text { associations }\end{array}$ \\
\hline $\begin{array}{l}\text { Turati, } 2016 \text { (13); Case-Control Study; Italy; } \\
\text { Atopic Dermatitis Cases: 451, Controls: } 451\end{array}$ & $\begin{array}{l}\text { Age of nut/cacao/chocolate introduction: }<7 \text { vs. } \\
>7 \mathrm{mo}\end{array}$ & $\begin{array}{l}\text { Atopic dermatitis at } 24 \mathrm{mo} \text { : No significant } \\
\text { associations }\end{array}$ \\
\hline $\begin{array}{l}\text { Venter, } 2016 \text { (12); Nested Case-Control } \\
\text { Study (Food Allergy and Intolerance } \\
\text { Research Cohort); UK; Food Allergy Cases: } \\
\text { 39, Controls: } 78\end{array}$ & $\begin{array}{l}\text { Age of peanut introduction: }<3,3-6,6-9, \text { vs. 9- } \\
12 \text { mo }\end{array}$ & Food allergy at 1y: No significant associations \\
\hline $\begin{array}{l}\text { Yu, } 2011 \text { (14); Case-Control Study; US; } \\
\text { Allergic Cases: 67, Non-Allergic Controls: } \\
191\end{array}$ & $\begin{array}{l}\text { Age of common nut introduction: } 0-12,13-24 \text {, } \\
25-36 \text {, vs. }>36 \text { mo }\end{array}$ & $\begin{array}{l}\text { Food allergy at 14-18y: No significant } \\
\text { associations }\end{array}$ \\
\hline $\begin{array}{l}\text { Zutavern, } 2006 \text { (8); Prospective Cohort } \\
\text { Study (Lifestyle-Related Factors on the } \\
\text { Immune System and the Development of } \\
\text { Allergies in Childhood Study); Germany; N: } \\
2612\end{array}$ & $\begin{array}{l}\text { Age of soybean/nut/cacao/chocolate } \\
\text { introduction: } 0-4,5-6 \text {, vs. }>6 \text { mo }\end{array}$ & $\begin{array}{l}\text { Atopic dermatitis at } 2 \mathrm{y} \text { : No significant } \\
\text { associations }\end{array}$ \\
\hline
\end{tabular}


Table 4. Results of studies that examined the relationship between egg consumption during the complementary feeding period and risk of atopic disease.

\begin{tabular}{|c|c|c|}
\hline $\begin{array}{l}\text { Reference; Study design (Study or cohort } \\
\text { name); Country; Sample size }\end{array}$ & Intervention or exposure & Results \\
\hline \multicolumn{3}{|l|}{ Randomized Controlled Trials } \\
\hline \multirow{2}{*}{$\begin{array}{l}\text { Bellach, } 2016 \text { (15); Randomized Controlled } \\
\text { Trial (Hen's Egg Allergy Prevention Study } \\
\text { (HEAP); Germany; Egg-powder Group: 124, } \\
\text { Rice-powder Group: } 152\end{array}$} & $\begin{array}{l}\text { Egg group: Pasteurized (uncooked) egg-white } \\
\text { powder ( 1/3 egg, } 3 \text { times/wk) from 4-6mo to } \\
\text { 12mo }\end{array}$ & $\begin{array}{l}\text { Egg allergy at 12mo: No significant group } \\
\text { differences }\end{array}$ \\
\hline & $\begin{array}{l}\text { Rice group: Rice powder and an egg-free diet } \\
\text { from } 4-6 \text { mo to } 12 \mathrm{mo}\end{array}$ & \\
\hline \multirow{2}{*}{$\begin{array}{l}\text { Natsume, } 2017 \text { (16); Randomized Controlled } \\
\text { Trial (Prevention of Egg Allergy with Tiny } \\
\text { Amount Intake Study); Japan; Egg Group: } \\
\text { 74, Placebo Group: } 73\end{array}$} & $\begin{array}{l}\text { Egg group: Cooked whole egg powder (0.2- } \\
1.1 \mathrm{~g} / \mathrm{d} \text { egg) from } 6 \text { to } 12 \mathrm{mo}\end{array}$ & $\begin{array}{l}\text { Egg allergy at } 1 \mathrm{y}: 8 \% \text { vs. } 38 \% ; \mathrm{RR}=0.221 \\
95 \% \mathrm{Cl}: 0.090,0.543 ; \mathrm{P}=0.0001\end{array}$ \\
\hline & $\begin{array}{l}\text { Placebo group: Squash powder and an egg- } \\
\text { free diet from } 6 \text { to } 12 \mathrm{mo}\end{array}$ & \\
\hline \multirow{2}{*}{$\begin{array}{l}\text { Palmer, } 2013 \text { (17); Randomized Controlled } \\
\text { Trial; Australia; Egg group: 42, Rice Group: } \\
35\end{array}$} & $\begin{array}{l}\text { Egg group: Pasteurized raw whole egg } \\
\text { powder }(\sim 1 / 6 \text { egg/d) from } 4-6 \text { to } 10 \mathrm{mo}\end{array}$ & $\begin{array}{l}\text { Egg allergy at } 8 \mathrm{mo} \text { and } 12 \mathrm{mo} \text { : No significant } \\
\text { group differences }\end{array}$ \\
\hline & $\begin{array}{l}\text { Rice group: Rice flour powder and egg-free } \\
\text { diet from } 4-6 \text { to } 10 \mathrm{mo}\end{array}$ & \\
\hline \multirow{2}{*}{$\begin{array}{l}\text { Palmer, } 2016 \text { (18); Randomized Controlled } \\
\text { Trial (Starting Time of Egg Protein Trial } \\
\text { (STEP); Australia; Egg Group: 371, Egg-free } \\
\text { Group: } 377\end{array}$} & $\begin{array}{l}\text { Egg group: Received } 0.9 \mathrm{~g} / \mathrm{d} \text { pasteurized raw } \\
\text { whole egg powder }(0.4 \mathrm{~g} / \mathrm{d} \text { egg protein, } \sim 1 / 2 \\
\text { egg/wk) from } 4-6 \text { to } 10 \mathrm{mo}\end{array}$ & $\begin{array}{l}\text { Egg allergy at } 12 \mathrm{mo} \text { : No significant group } \\
\text { differences }\end{array}$ \\
\hline & $\begin{array}{l}\text { Control group: Rice powder and egg-free diet } \\
\text { from } 4-6 \text { to } 10 \mathrm{mo}\end{array}$ & \\
\hline $\begin{array}{l}\text { Perkin, } 2016 \text { (2); Randomized Controlled } \\
\text { Trial (Enquiring about Tolerance Trial (EAT); } \\
\text { UK; Standard Introduction Group: 595, Early } \\
\text { Introduction Group: } 567\end{array}$ & $\begin{array}{l}\text { Early introduction group: Introduction to } \\
\text { allergenic foods at } 3 \text { mo (cow's milk (yogurt) } \\
\text { first, then (in random order) peanut, cooked }\end{array}$ & $\begin{array}{l}\text { Food allergy from 1-3y, intention to treat } \\
\text { analyses: No significant group differences } \\
\text { based on intention to treat analyses }\end{array}$ \\
\hline
\end{tabular}




\begin{tabular}{|c|c|c|}
\hline \multirow[t]{5}{*}{$\begin{array}{l}\text { Reference; Study design (Study or cohort } \\
\text { name); Country; Sample size }\end{array}$} & Intervention or exposure & Results \\
\hline & $\begin{array}{l}\text { (boiled) hen's egg, sesame, and whitefish, and } \\
\text { wheat last) }\end{array}$ & \\
\hline & $\begin{array}{l}\text { Standard introduction group: Introduction to } \\
\text { allergenic foods at } 6 \text { mo according to parental } \\
\text { discretion }\end{array}$ & $\begin{array}{l}\text { Food allergy from } 1-3 y \text {, per protocol analyses: } \\
2.4 \% \text { vs. } 6.4 \% ; P=0.03\end{array}$ \\
\hline & & $\begin{array}{l}\text { Egg allergy from } 1-3 y \text {, per protocol analyses: } \\
1.4 \% \text { vs. } 5.2 \% ; P=0.02\end{array}$ \\
\hline & $\begin{array}{l}\text { Amount of egg consumed: } 4 \mathrm{vs} .<4 \mathrm{~g} / \mathrm{wk} \text { from } 3 \\
\text { to } 6 \mathrm{mo} \text { (early introduction group only) }\end{array}$ & $\begin{array}{l}\text { Egg allergy from 1-3y: Reduced risk with } \\
4 \mathrm{~g} / \mathrm{wk} \text { vs. }<4 \mathrm{~g} / \mathrm{wk} ; \mathrm{P}<0.05\end{array}$ \\
\hline \multirow{2}{*}{$\begin{array}{l}\text { Wei-Liang Tan, } 2016 \text { (19); Randomized } \\
\text { Controlled Trial (Beating Egg Allergy Trial } \\
\text { (BEAT); Australia; Egg Group: 130, Rice } \\
\text { Group: } 124\end{array}$} & $\begin{array}{l}\text { Egg powder: Pasteurized whole egg (raw) } \\
\text { powder }(350 \mathrm{mg} / \mathrm{d}) \text { from } 4 \text { to } 8 \mathrm{mo}\end{array}$ & $\begin{array}{l}\text { Egg allergy at 12mo: No significant group } \\
\text { differences }\end{array}$ \\
\hline & $\begin{array}{l}\text { Rice powder: Rice powder and egg-free diet } \\
\text { from } 4 \text { to } 8 \mathrm{mo}\end{array}$ & $\begin{array}{l}\text { Atopic dermatitis at 12mo: No significant group } \\
\text { differences }\end{array}$ \\
\hline \multicolumn{3}{|l|}{ Observational Studies } \\
\hline $\begin{array}{l}\text { Alm, } 2011 \text { (20); Prospective Cohort Study } \\
\text { (Infants of Western Sweden); Sweden; N: } \\
4496\end{array}$ & $\begin{array}{l}\text { Early egg introduction: Not defined (univariate } \\
\text { analyses) }\end{array}$ & $\begin{array}{l}\text { Allergic rhinitis at } 4.5 y \text { : No significant } \\
\text { associations }\end{array}$ \\
\hline $\begin{array}{l}\text { Bisgaard, } 2009 \text { (21); Prospective Cohort } \\
\text { Study; Denmark (Copenhagen Study on } \\
\text { Asthma in Childhood); Denmark; N:356 }\end{array}$ & $\begin{array}{l}\text { Age of egg introduction: Duration in days } \\
\text { (continuous) }\end{array}$ & $\begin{array}{l}\text { Atopic dermatitis at } 3 y \text { : No significant } \\
\text { associations }\end{array}$ \\
\hline \multirow{2}{*}{$\begin{array}{l}\text { Fergusson, } 1981 \text { (22); Prospective Cohort } \\
\text { Study (Christchurch Health and } \\
\text { Development Study); New Zealand; N: } 1156\end{array}$} & $\begin{array}{l}\text { Consumption of egg: Presence vs. absence at } \\
4 \mathrm{mo} \text {; }\end{array}$ & $\begin{array}{l}\text { Atopic dermatitis at 2y: No significant } \\
\text { associations }\end{array}$ \\
\hline & $\begin{array}{l}\text { Amount of egg consumed: Teaspoonful/d at } \\
4 \mathrm{mo}\end{array}$ & $\begin{array}{l}\text { Atopic dermatitis at 2y: No significant } \\
\text { associations }\end{array}$ \\
\hline $\begin{array}{l}\text { Filipiak, } 2007 \text { (3); Prospective Cohort Study } \\
\text { (German Infant Nutritional Intervention } \\
\text { Program); Germany; N: } 4753\end{array}$ & Age of egg introduction: $>12$ vs. $<12 \mathrm{mo}$ & $\begin{array}{l}\text { Atopic dermatitis, doctor-diagnosed, at } 4 \mathrm{y} \text { : } \\
\mathrm{OR}=1.80,95 \% \mathrm{Cl}: 1.23,2.64 ; \mathrm{P}<0.05\end{array}$ \\
\hline
\end{tabular}




\begin{tabular}{|c|c|c|}
\hline $\begin{array}{l}\text { Reference; Study design (Study or cohort } \\
\text { name); Country; Sample size }\end{array}$ & Intervention or exposure & Results \\
\hline & & $\begin{array}{l}\text { Atopic dermatitis, symptomatic, at 4y: No } \\
\text { significant associations }\end{array}$ \\
\hline $\begin{array}{l}\text { Grimshaw, } 2013 \text { (10); Nested Case-Control } \\
\text { Study (Prevalence of Infant Food Allergy } \\
\text { EuroPrevall); UK; Food Allergy Cases: 41, } \\
\text { Controls: } 82\end{array}$ & Age of hen's egg introduction: Median in wk & Food allergy at 2y: No significant associations \\
\hline $\begin{array}{l}\text { Grimshaw, } 2015 \text { (9); Nested Case-Control } \\
\text { Study (Prevalence of Infant Food Allergy } \\
\text { EuroPrevall); UK; Food Allergy Cases: 41, } \\
\text { Controls: } 82\end{array}$ & Age of egg introduction: Median in mo & $\begin{array}{l}\text { Food allergy, IgE-mediated, at 2y: OR=1.05, } \\
95 \% \mathrm{Cl}: 1.00,1.11 ; \mathrm{P}=0.026\end{array}$ \\
\hline \multirow[t]{3}{*}{$\begin{array}{l}\text { Gustafsson, } 2000 \text { (23); Prospective Cohort } \\
\text { Study; Sweden; N:94 }\end{array}$} & Age of hen's egg introduction: $<12$ vs. $>12$ mo & $\begin{array}{l}\text { Atopic dermatitis through 8y: No significant } \\
\text { associations }\end{array}$ \\
\hline & & Asthma through 8y: No significant associations \\
\hline & & $\begin{array}{l}\text { Allergic rhinitis through 8y: No significant } \\
\text { associations }\end{array}$ \\
\hline \multirow[t]{2}{*}{$\begin{array}{l}\text { Hesselmar, } 2010 \text { (24); Prospective Cohort } \\
\text { Study (ALLERGY-FLORA); Sweden; N: } 184\end{array}$} & $\begin{array}{l}\text { Age of hen's egg introduction: Months, } \\
\text { continuous }\end{array}$ & $\begin{array}{l}\text { Food allergy at 18mo: No significant } \\
\text { associations }\end{array}$ \\
\hline & & $\begin{array}{l}\text { Atopic dermatitis at 18mo: No significant } \\
\text { associations }\end{array}$ \\
\hline \multirow{4}{*}{$\begin{array}{l}\text { Kumar, } 2010 \text { (11); Nested Case-Control } \\
\text { Study; US; Food Allergy Cases: 411, } \\
\text { Asymptomatic Sensitized Cases: 171, } \\
\text { Controls: } 378\end{array}$} & $\begin{array}{l}\text { Age of egg, peanut, tree nut, shellfish, fish, } \\
\text { and sesame (combined) introduction: }>6 \text { vs. } \\
<6 \text { mo }\end{array}$ & $\begin{array}{l}\text { Food allergy at } \sim 6 y: \text { OR=0.8, } 95 \% \mathrm{Cl}: 0.4,1.6 \text {; } \\
\mathrm{P}<0.05)\end{array}$ \\
\hline & & $\begin{array}{l}\text { Food allergy at } \sim 6 y \text {, children without atopic } \\
\text { dermatitis: No significant associations }\end{array}$ \\
\hline & & $\begin{array}{l}\text { Food allergy at } \sim 6 y, \text { children with atopic } \\
\text { dermatitis: No significant associations }\end{array}$ \\
\hline & $\begin{array}{l}\text { Age of egg, peanut, tree nut, shellfish, fish, } \\
\text { and sesame (combined) introduction: }>1 \text { vs. } \\
<1 y\end{array}$ & $\begin{array}{l}\text { Food allergy at } \sim 6 y: \text { No significant } \\
\text { associations }\end{array}$ \\
\hline
\end{tabular}




\section{Reference; Study design (Study or cohort Intervention or exposure name); Country; Sample size}

\section{Results}

Food allergy at $\sim 6 y$, children without atopic dermatitis: $\mathrm{OR}=0.5,95 \% \mathrm{Cl}$ : 0.3, 0.95; $\mathrm{P}<0.05$

Food allergy at $\sim 6 y$, children with atopic dermatitis: No significant associations

Niinivirta, 2014 (25); Prospective Cohort Study; Finland; N: 223 at $6 \mathrm{mo} ; 211$ at $12 \mathrm{mo}$; 185 at $24 \mathrm{mo} ; 129$ at $48 \mathrm{mo}$

Nwaru, 2013 (27); Prospective Cohort Study; Age of egg introduction: 8, 8-11 vs. >11mo Finland (Finnish Type 1 Diabetes Prediction and Prevention Study); Finland; N: 3781

Atopic dermatitis through 4y: No significant associations

Atopic dermatitis at 5y: No significant associations
Age of egg introduction: 6 vs. $\geq 7 \mathrm{mo}$

Asthma, total, at $5 \mathrm{y}: \mathrm{HR}=0.61,95 \% \mathrm{Cl}: 0.39$, 0.94 ; $\mathrm{HR}=0.55,95 \% \mathrm{Cl}: 0.38,0.81 ; \mathrm{P}=0.005$ _

Asthma, atopic, at $5 \mathrm{y}: \mathrm{HR}=0.46,95 \% \mathrm{Cl}: 0.25$, 0.84; $\mathrm{HR}=0.55,95 \% \mathrm{Cl}: 0.34,0.91 ; \mathrm{P}<0 . .001$

Allergic rhinitis at $5 \mathrm{y}: \mathrm{HR}=0.73,95 \% \mathrm{Cl}: 0.52$, 1.02; $\mathrm{HR}=0.72,95 \% \mathrm{Cl}: 0.55,0.94 ; \mathrm{P}=0.04$

\begin{tabular}{lll}
\hline Nwaru, 2013 (26); Prospective Cohort Study & Age of egg introduction: $<5$ vs. $>5$ mo & Atopic dermatitis at 10y: No significant \\
(Study of Atopic dermatitis and Asthma To & & associations \\
Observe the Influence of Nutrition); UK; N: & &
\end{tabular}

934
Allergic rhinitis at 10y: No significant associations

Atopic dermatitis at 1y: OR=0.55, 95\% Cl: $0.38,0.80 ; P<0.05$

Age of egg introduction: 3-12 vs. >12mo (Protection Against Allergy in Rural

Environments Study); Austria, Finland,

France, Germany, Switzerland; N: 1041

Roduit, 2014 (5); Prospective Cohort Study

(Protection Against Allergy in Rural

Environments Study); Austria, Finland,

France, Germany, Switzerland; N: 856
Age of egg introduction: $<12 \mathrm{mo}$ vs. Never

Food allergy at 6y: No significant associations

Asthma at 6y: No significant associations 


\begin{tabular}{|c|c|c|}
\hline $\begin{array}{l}\text { Reference; Study design (Study or cohort } \\
\text { name); Country; Sample size }\end{array}$ & Intervention or exposure & Results \\
\hline $\begin{array}{l}\text { Sariachvili, } 2010 \text { (30); Nested Case-Control } \\
\text { Study (Influence of Perinatal Factors on the } \\
\text { Occurrence of Asthma and Allergy Study); } \\
\text { Belgium; atopic dermatitis cases: } 252 \text {, } \\
\text { Controls: } 305\end{array}$ & Age of egg introduction: $<4$ vs. $>4 \mathrm{mo}$ & Atopic dermatitis: No significant associations \\
\hline $\begin{array}{l}\text { Tromp, } 2011 \text { (7); Prospective Cohort Study } \\
\text { (Generation R Study); The Netherlands; N: } \\
6905\end{array}$ & Age of hen's egg introduction: Not defined & $\begin{array}{l}\text { Atopic dermatitis at 2, 3, and 4y: No significant } \\
\text { associations }\end{array}$ \\
\hline $\begin{array}{l}\text { Turati, } 2016 \text { (13); Case-Control Study; Italy; } \\
\text { Atopic Dermatitis Cases: 451, Controls: } 451\end{array}$ & Age of egg introduction: $<8$ vs. $>8 \mathrm{mo}$ & $\begin{array}{l}\text { Atopic dermatitis at } 24 \mathrm{mo} \text { : No significant } \\
\text { associations }\end{array}$ \\
\hline $\begin{array}{l}\text { Venter, } 2016 \text { (12); Nested Case-Control } \\
\text { Study (Food Allergy and Intolerance } \\
\text { Research Cohort); UK; Food Allergy Cases: } \\
\text { 39. Controls: } 78\end{array}$ & $\begin{array}{l}\text { Age of egg introduction: }<3,3-6,6-9, \text { vs. } 9- \\
12 \mathrm{mo}\end{array}$ & Food allergy at 1y: No significant associations \\
\hline \multirow[t]{2}{*}{$\begin{array}{l}\text { Virtanen, } 2010 \text { (28); Prospective Cohort } \\
\text { Study (Diabetes Prediction and Prevention } \\
\text { Study); Finland; N: } 1288\end{array}$} & Age of egg introduction: $<8,8.5-11,>11 \mathrm{mo}$ & Asthma at 5y: No significant associations \\
\hline & & $\begin{array}{l}\text { Allergic rhinitis at } 5 y \text { : No significant } \\
\text { associations }\end{array}$ \\
\hline $\begin{array}{l}\text { Yu, } 2011 \text { (14); Case-Control Study; US; } \\
\text { Allergic Cases: 67, Non-Allergic Controls: } \\
191\end{array}$ & $\begin{array}{l}\text { Age of egg introduction: } 0-12,13-24,25-36 \text {, } \\
\text { vs. }>36 \mathrm{mo}\end{array}$ & $\begin{array}{l}\text { Food allergy at } 14-18 \mathrm{y}: \mathrm{OR}=0.15,95 \% \mathrm{Cl} \text { : } \\
0.18,0.27 ; \mathrm{P}<0.01\end{array}$ \\
\hline $\begin{array}{l}\text { Zutavern, } 2004 \text { (29); Prospective Cohort } \\
\text { Study; UK; N: } 552\end{array}$ & Age of egg introduction: $>8$ vs. $<8 \mathrm{mo}$ & $\begin{array}{l}\text { Atopic dermatitis at } 5 \mathrm{y}: \mathrm{OR}=1.6,95 \% \mathrm{Cl}: 1.1 \text {, } \\
2.4 ; \mathrm{P}<0.05\end{array}$ \\
\hline $\begin{array}{l}\text { Zutavern, } 2006 \text { (8); Prospective Cohort } \\
\text { Study (Lifestyle-Related Factors on the } \\
\text { Immune System and the Development of } \\
\text { Allergies in Childhood Study); Germany; } \\
\text { N:2612 }\end{array}$ & Age of egg introduction: $0-4,5-6$, vs. $>6 \mathrm{mo}$ & $\begin{array}{l}\text { Atopic dermatitis at 5y: No significant } \\
\text { associations }\end{array}$ \\
\hline
\end{tabular}


Table 5. Results of studies that examined the relationship between fish and/or shellfish consumption during the complementary feeding period and risk of atopic disease.

\begin{tabular}{|c|c|c|}
\hline $\begin{array}{l}\text { Reference; Study design (Study or } \\
\text { cohort name); Country; Sample size }\end{array}$ & Intervention or exposure & Results \\
\hline \multirow[t]{4}{*}{$\begin{array}{l}\text { Alm, } 2009 \text { (31); Prospective Cohort Study } \\
\text { (Infants of Western Sweden); Sweden; N: } \\
4921\end{array}$} & $\begin{array}{l}\text { Age of fish introduction: }<9 \text { vs. }>9 \text { mo } \\
\text { (multivariate analyses); } 0-2,3-5 \text {, or } 6-8 \text { vs .9- } \\
12 \text { mo (univariate analyses) }\end{array}$ & $\begin{array}{l}\text { Atopic dermatitis at } 12 \mathrm{mo}: \mathrm{OR}=0.76,95 \% \mathrm{Cl} \text { : } \\
0.62,0.94 ; \mathrm{P}=0.009\end{array}$ \\
\hline & & $\begin{array}{l}\text { Atopic dermatitis at } 12 \mathrm{mo}: 3-5 \text { vs } 9-12 \mathrm{mo}: \\
\text { OR=0.7, 95\% Cl: } 0.6,0.9 ; \mathrm{P}=0.003 ; 6-8 \text { vs } \\
\text { 912mo: OR=0.6, } 95 \% \mathrm{Cl}: 0.5,0.7 ; \mathrm{P}<0.001\end{array}$ \\
\hline & $\begin{array}{l}\text { Frequency of fish consumption: Never, a few } \\
\text { times a year, } 1-3 / \mathrm{mo}, 1-3 / \mathrm{wk} \text {, vs. 3+/wk } \\
\text { (univariate analyses) }\end{array}$ & $\begin{array}{l}\text { Atopic dermatitis at 12mo: Never vs. } 3+/ w k: \\
\text { OR=2.73, } 95 \% \mathrm{Cl}: 1.80,4.13 ; \mathrm{P}<0.001\end{array}$ \\
\hline & $\begin{array}{l}\text { Type of fish consumed: lean fish (cod, } \\
\text { haddock) vs. other fish (salmon, flatfish, } \\
\text { mackerel, herring) (univariate analyses) }\end{array}$ & $\begin{array}{l}\text { Atopic dermatitis at } 12 \mathrm{mo}: \mathrm{OR}=0.81,95 \% \mathrm{Cl} \text { : } \\
0.68,0.97 ; \mathrm{P}=0.025\end{array}$ \\
\hline \multirow{3}{*}{$\begin{array}{l}\text { Alm, } 2011(20) ; \text { Prospective Cohort Study } \\
\text { (Infants of Western Sweden); Sweden; N: } \\
4496\end{array}$} & Age of fish introduction: $<9$ vs. $>9 \mathrm{mo}$ & $\begin{array}{l}\text { Allergic rhinitis at } 4.5 y: O R=0.49,95 \% \mathrm{Cl}: 0.29 \text {, } \\
0.82 ; \mathrm{P}=0.007\end{array}$ \\
\hline & $\begin{array}{l}\text { Frequency of fish consumption from } 0-12 \mathrm{mo} \text { : } \\
>1 \mathrm{x} / \mathrm{mo} \text { vs. }<1 \mathrm{x} / \mathrm{mo}\end{array}$ & $\begin{array}{l}\text { Allergic rhinitis at } 4.5 y \text { : No significant } \\
\text { associations }\end{array}$ \\
\hline & $\begin{array}{l}\text { Type of fish consumed: lean fish, fatty fish; } \\
\text { not defined }\end{array}$ & $\begin{array}{l}\text { Allergic rhinitis at 4.5y: No significant } \\
\text { associations }\end{array}$ \\
\hline $\begin{array}{l}\text { Bisgaard, } 2009 \text { (21); Prospective Cohort } \\
\text { Study (Copenhagen Study on Asthma in } \\
\text { Childhood); Denmark; N: } 356\end{array}$ & Age of fish introduction: Duration in days & $\begin{array}{l}\text { Atopic dermatitis at 3y: No significant } \\
\text { associations }\end{array}$ \\
\hline $\begin{array}{l}\text { Filipiak, } 2007 \text { (3); Prospective Cohort Study } \\
\text { (German Infant Nutritional Intervention } \\
\text { Program); Germany; N: } 4753\end{array}$ & Age of fish introduction: $1-4,5-6$, vs. $7-12 \mathrm{mo}$ & $\begin{array}{l}\text { Atopic dermatitis at } 4 \mathrm{y} \text { : No significant } \\
\text { associations }\end{array}$ \\
\hline $\begin{array}{l}\text { Goksor, } 2013 \text { (32); Prospective Cohort } \\
\text { Study (Infants of Western Sweden); } \\
\text { Sweden; N: } 4051\end{array}$ & Age of fish introduction: $<9$ vs. $>9 \mathrm{mo}$ & Asthma at 8y: OR=0.6, 95\% Cl: $0.4,0.96 ; \mathrm{P}<.05$ \\
\hline
\end{tabular}




\begin{tabular}{ll}
\hline \hline $\begin{array}{l}\text { Reference; Study design (Study or } \\
\text { cohort name); Country; Sample size }\end{array}$ & Intervention or exposure \\
\hline & \\
& \\
\hline & Frequency of fish consumption: $1+$ time/mo \\
vs. 0 time/mo at $1 \mathrm{y}$
\end{tabular}

Grimshaw, 2013 (10); Nested Case-Control Age of fish introduction: Median in wk

Study (Prevalence of Infant Food Allergy

EuroPrevall); UK; Food Allergy Cases: 41,

Controls: 82

\section{Results}

Atopic asthma at $8 y$ : OR=0.5, 95\% Cl: 0.3, 0.8; $P<0.95$ at $8 y$

Non-atopic asthma at 8y: No significant associations

Asthma (total, atopic, non-atopic) at 8y: No significant associations

Food allergy at 2y: No significant associations
Gustafsson, 2000 (23); Prospective Cohort

Study; Sweden; N: 94
Age of fish introduction: $<7$ vs. $>7 \mathrm{mo}$
Atopic dermatitis from 4mo-8y: No significant associations

Asthma from 4mo-8y: No significant associations

Allergic rhinitis from 4mo-8y: No significant associations
Hesselmar, 2010 (24); Prospective Cohort Age of fish introduction: Median mo for no

Study (ALLERGY-FLORA); Sweden; N: 184

Food allergy at 18mo: 9 vs. $13 \mathrm{mo} ; \mathrm{P}=0.01$

diagnosis vs. diagnosed

Age of egg, peanut, tree nut, shellfish, fish, and sesame (combined) introduction: $>6$ vs. $<6 \mathrm{mo}$

Atopic dermatitis at $18 \mathrm{mo}: 8$ vs. $11 \mathrm{mo} ; \mathrm{P}=0.004$

Kumar, 2010 (11); Nested Case-Control

Study; US; Food Allergy Cases: 411,

Asymptomatic Sensitized Cases: 171,

Controls: 378

Food allergy at $\sim 6 y$ : OR=0.8, 95\% Cl: 0.4, 1.6;

$\mathrm{P}<0.05)$
Food allergy at $\sim 6 y$, children without atopic dermatitis: No significant associations

Food allergy at $\sim 6 y$, children with atopic dermatitis: No significant associations

Age of egg, peanut, tree nut, shellfish, fish, and sesame (combined) introduction: $>1$ vs.

$<1 \mathrm{y}$
Food allergy at $\sim 6 y$ : No significant associations 


\section{Reference; Study design (Study or cohort name); Country; Sample size}

Intervention or exposure
Results

Food allergy at $\sim 6 y$, children without atopic dermatitis: $\mathrm{OR}=0.5,95 \% \mathrm{Cl}: 0.3,0.95 ; \mathrm{P}<0.05$

Food allergy at $\sim 6 y$, children with atopic dermatitis: No significant associations

Kull, 2006 (33); Prospective Cohort Study; $\quad$ Age of fish introduction: 3-8 vs. >9mo Sweden; N: 3619

Nafstad, 2003 (34); Prospective Cohort

Age of fish introduction: $<12$ vs. $>12$ mo

Frequency of fish consumption: 1/mo, 2 $3 / \mathrm{mo}, 1 / \mathrm{wk}, \geq 1 / \mathrm{wk}$ vs. Never
Atopic dermatitis at 4y: OR=0.77, 95\% Cl: 0.64, $0.92 ; \mathrm{P}<0.001$

Asthma at $4 \mathrm{y}$ : OR=0.73, 95\% Cl: 0.55, 0.97; $\mathrm{P}<0.001$

Allergic rhinitis at $4 \mathrm{y}: \mathrm{OR}=0.77,95 \% \mathrm{Cl}: 0.60$, $0.97 ; \mathrm{P}<0.001$

Atopic dermatitis at 4y: $1 / \mathrm{mo}: \mathrm{OR}=0.72,95 \% \mathrm{Cl}$ : 0.51, 1.00; 2-3/mo: OR=0.71, 95\% Cl: 0.53, 0.95; 1/wk: OR=0.54, 95\% Cl: 0.41, 0.70; $\geq 1 / \mathrm{wk}$ : $\mathrm{OR}=0.57,95 \% \mathrm{Cl}: 0.43,0.76 ; \mathrm{P}<0.001$

Asthma at 4y: 1/mo: OR=0.94, 95\% Cl: 0.57 , 1.56; 2-3/mo: OR=0.82, 95\% Cl: 0.54, 1.29; 1/wk: OR=0.66, 95\% Cl: $0.43,1.01 ; \geq 1 / w k$ : $\mathrm{OR}=0.55,95 \% \mathrm{Cl}: 0.34,0.87 ; \mathrm{P}<0.001$

Allergic rhinitis at $4 \mathrm{y}: 1 / \mathrm{mo}: \mathrm{OR}=0.52,95 \% \mathrm{Cl}$ : 0.34, 0.79; 2-3/mo: OR=0.51, 95\% Cl: 0.35, 0.73; $\mathrm{OR}=0.34,95 \% \mathrm{Cl}: 0.24,0.49 ; \mathrm{P}<0.001$

Atopy at 4y: 1/mo: OR=0.61, 95\% Cl: 0.45, 0.84; 2-3/mo: OR=0.58, 95\% Cl: $0.45,0.76 ; 1 / \mathrm{wk}$ : $\mathrm{OR}=0.50,95 \% \mathrm{Cl}: 0.39,0.64 ; \geq 1 / \mathrm{wk}: \mathrm{OR}=0.46$; , 95\% Cl: 0.35, 0.60; $\mathrm{P}<0.001$

Atopic dermatitis at 4y: OR=0.66, $95 \% \mathrm{Cl}: 0.52$ $0.84 ; P=0.001$

Asthma at 4y: No significant associations Allergic rhinitis at $4 \mathrm{y}: \mathrm{OR}=0.45,95 \% \mathrm{Cl}: 0.28$, 0.74 1/wk: OR=0.47, 95\% Cl: 0.32, 0.62; $\geq 1 / w k$ : 


\section{Reference; Study design (Study or cohort name); Country; Sample size}

Intervention or exposure

Results

Allergic rhinitis at $4 \mathrm{y}$, in those $\mathrm{BF}>6 \mathrm{mo}$ : $\mathrm{OR}=0.28,95 \% \mathrm{Cl}: 0.15,0.52$

Allergic rhinitis at $4 \mathrm{y}$, in those with atopic dermatitis <6mo: OR=0.32, 95\% Cl: 0.15, 0.69

Allergic rhinitis at $4 \mathrm{y}$, in those without respiratory infection <12mo: OR=0.39, 95\% Cl: 0.24, 0.66

Niinivirta, 2014 (25); Prospective Cohort Study; Finland; N: 223 at 6mo; 211 at 12mo;

Age of fish introduction: 6 vs. $\geq 7 \mathrm{mo}$ 185 at $24 \mathrm{mo} ; 129$ at $48 \mathrm{mo}$

Nwaru, 2013 (27); Prospective Cohort Study Age of fish introduction: 6, 6-9 vs >9mo (Finnish Type 1 Diabetes Prediction and Prevention Study); Finland; N: 3781

Atopic dermatitis from $6 \mathrm{mo}-4 \mathrm{y}$ : No significant association

Atopic dermatitis at 5y: No significant associations

Asthma at 5y: No significant associations

Allergic rhinitis at 5y: No significant associations

Nwaru, 2013 (26); Prospective Cohort Study Age of fish introduction: Categorized by

(Study of Eczema and Asthma To Observe tertiles, based on month of introduction the Influence of Nutrition); UK; N: 934

Atopic dermatitis at 10y: No significant

associations

Allergic rhinitis at 10y: No significant associations

Perkin, 2016 (2); Randomized Controlled Trial (Enquiring about Tolerance Trial

(EAT); UK; Standard Introduction Group: 595, Early Introduction Group: 567 first, then (in random order) peanut, cooked
Early introduction group: Introduction to allergenic foods at 3mo (cow's milk (yogurt) (boiled) hen's egg, sesame, and whitefish, and wheat last)

Standard introduction group: Introduction to allergenic foods at $6 \mathrm{mo}$ according to parental discretion
Food allergy from 1-3y, intention to treat analyses: No significant group differences based on intention to treat analyses

Food allergy from 1-3y, per protocol analyses: $2.4 \%$ vs. $6.4 \% ; P=0.03$

Fish allergy from 1-3y, per protocol analyses: No significant group differences 


\section{Reference; Study design (Study or cohort name); Country; Sample size}

Oien, 2010 (35); Prospective Cohort Study (Prevention of Allergy Among Children in

Trondheim Study); Norway; N: 3086
Intervention or exposure

Frequency of fish consumption: $>1$ time/wk vs. $<1$ time/wk, Never

Frequency of oily fish (redfish, halibut, salmon, trout, herring, mackerel) consumption: Never, $>1$ time/wk consumption: Never, $>1$ time/wk

\section{Results}

Atopic dermatitis at $2 \mathrm{y}: \mathrm{OR}=0.66,95 \% \mathrm{Cl}: 0.53$, $0.81, P<0.001$, which persisted when early onset Atopic dermatitis $(<1 \mathrm{y})$ children were excluded $(P=0.02)$

Asthma at 2y: No significant associations

Atopic dermatitis at 2y: OR=0.57, 95\% Cl: 0.35 $0.94, \mathrm{P}=0.03$, which persisted when early onset atopic dermatitis $(<1 \mathrm{y})$ children were excluded $(\mathrm{P}=0.03)$

Asthma at 2y: No significant associations

Atopic dermatitis at 2y: OR=0.66, 95\% Cl: 0.50 $0.86, \mathrm{P}<0.001$, though, this was no longer significant when early onset atopic dermatitis $(<1 \mathrm{y})$ children were excluded (NS)

Atopic dermatitis at $2 y$, without early onset atopic dermatitis (<1y) children: No significant associations

Asthma at 2y: No significant associations

Frequency of cod liver oil consumption:

Never, $<3$, >4 times/w
Atopic dermatitis at 2y: No significant associations

Asthma at 2y: No significant associations

(Protection Against Allergy in Rural

Age of fish introduction: $3-12$ vs. $>12 \mathrm{mo}$

Atopic dermatitis at 4y: OR=0.52; 0.35, 0.77; $P<0.05$

Environments Study); Austria, Finland,

France, Germany, Switzerland; N: 1041

Atopic dermatitis at $4 \mathrm{y}$, with no itchy rash by 6mo: $\mathrm{OR}=0.51,95 \% \mathrm{Cl}: 0.30,0.87 ; \mathrm{P}<0.05$

Roduit, 2014 (5); Prospective Cohort Study Age of fish introduction: <12mo vs. Never (Protection Against Allergy in Rural

Environments Study); Austria, Finland,

France, Germany, Switzerland; N: 856 


\begin{tabular}{|c|c|c|}
\hline $\begin{array}{l}\text { Reference; Study design (Study or } \\
\text { cohort name); Country; Sample size }\end{array}$ & Intervention or exposure & Results \\
\hline & & $\begin{array}{l}\text { Asthma at } 6 y: O R=0.41,95 \% \mathrm{Cl}: 0.19,0.88 \\
P<0.05\end{array}$ \\
\hline $\begin{array}{l}\text { Sariachvili, } 2010 \text { (30); Nested Case-Control } \\
\text { Study (Influence of Perinatal Factors on the } \\
\text { Occurrence of Asthma and Allergies Study); } \\
\text { Belgium; atopic dermatitis cases: } 252 \text {, } \\
\text { Controls: } 305\end{array}$ & Age of fish introduction: $<4$ vs. $>4 \mathrm{mo}$ & $\begin{array}{l}\text { Atopic dermatitis from 0-4y: No significant } \\
\text { associations }\end{array}$ \\
\hline $\begin{array}{l}\text { Turati, } 2016 \text { (13); Case-Control Study; Italy; } \\
\text { Atopic Dermatitis Cases: 451, Controls: } 451\end{array}$ & Age of fish introduction: $<7$ vs. $>7 \mathrm{mo}$ & $\begin{array}{l}\text { Atopic dermatitis at 24mo: No significant } \\
\text { associations }\end{array}$ \\
\hline \multirow{2}{*}{$\begin{array}{l}\text { Virtanen, } 2010 \text { (28); Prospective Cohort } \\
\text { Study (Diabetes Prediction and Prevention } \\
\text { Study); Finland; N: } 1288\end{array}$} & $\begin{array}{l}\text { Age of fish introduction:: Categorized by } \\
\text { tertiles, based on month of introduction }\end{array}$ & Asthma at 5y: No significant association \\
\hline & & $\begin{array}{l}\text { Allergic rhinitis at } 5 \mathrm{y}:<6 \text { vs. }>8.5 \mathrm{mo}: \mathrm{OR}=0.49 \text {, } \\
95 \% \mathrm{Cl}: 0.29,0.84 ; 6.1-8.5 \mathrm{vs} .>8.5 \mathrm{mo}: \\
\mathrm{OR}=0.91,95 \% \mathrm{Cl}: 0.56,1.48 ; \mathrm{P}=0.032)\end{array}$ \\
\hline $\begin{array}{l}\text { Yu, } 2011 \text { (14); Case-Control Study; US; } \\
\text { Allergic Cases: 67, Non-Allergic Controls: } \\
191\end{array}$ & $\begin{array}{l}\text { Age of shellfish introduction: } 0-12,13-24,25- \\
36, \text { vs. }>36 \mathrm{mo}\end{array}$ & $\begin{array}{l}\text { Food allergy at } 14-18 y \text { : No significant } \\
\text { associations }\end{array}$ \\
\hline $\begin{array}{l}\text { Zutavern, } 2004 \text { (29); Prospective Cohort } \\
\text { Study; UK; N: } 552\end{array}$ & Age of fish introduction: $<6$ vs. $>6 \mathrm{mo}$ & $\begin{array}{l}\text { Atopic dermatitis at 5y: No significant } \\
\text { associations }\end{array}$ \\
\hline $\begin{array}{l}\text { Zutavern, } 2006 \text { (8); Prospective Cohort } \\
\text { Study (Lifestyle-Related Factors on the } \\
\text { Immune System and the Development of } \\
\text { Allergies in Childhood Study); Germanyy; N: } \\
2612\end{array}$ & Age of fish introduction: $0-4,5-6$, vs. $>6 \mathrm{mo}$ & $\begin{array}{l}\text { Atopic dermatitis at 2y: No significant } \\
\text { associations }\end{array}$ \\
\hline
\end{tabular}


Table 6. Results of studies that examined the relationship between milk product consumption during the complementary feeding period and risk of atopic disease.

\begin{tabular}{|c|c|c|}
\hline $\begin{array}{l}\text { Reference; Study design (Study or cohort } \\
\text { name); Country; Sample size }\end{array}$ & Intervention or exposure & Results \\
\hline $\begin{array}{l}\text { Alm, } 2009 \text { (31); Prospective Cohort Study } \\
\text { (Infants of Western Sweden); Sweden; N: } \\
4921\end{array}$ & Type of fat on bread: Margarine vs. Dairy & $\begin{array}{l}\text { Atopic dermatitis at } 12 \mathrm{mo}: \mathrm{OR}=1.32,95 \% \mathrm{Cl} \text { : } \\
1.13,1.55 ; \mathrm{P}=0.001\end{array}$ \\
\hline $\begin{array}{l}\text { Alm, } 2011 \text { (20); Prospective Cohort Study } \\
\text { (Infants of Western Sweden); Sweden; N: } \\
4496\end{array}$ & Type of spread: Dairy vs. margarine & $\begin{array}{l}\text { Allergic rhinitis at } 4.5 y \text { : No significant } \\
\text { associations }\end{array}$ \\
\hline \multirow{2}{*}{$\begin{array}{l}\text { Fergusson, } 1981 \text { (22); Prospective Cohort } \\
\text { Study (Christchurch Health and Development } \\
\text { Study); New Zealand; N: } 1156\end{array}$} & $\begin{array}{l}\text { Consumption of dairy products: Presence vs. } \\
\text { absence at } 4 \mathrm{mo}\end{array}$ & $\begin{array}{l}\text { Atopic dermatitis at } 2 \mathrm{y} \text { : No significant } \\
\text { associations }\end{array}$ \\
\hline & $\begin{array}{l}\text { Amount of dairy products consumed: } \\
\text { Teaspoonful/d at } 4 \mathrm{mo}\end{array}$ & $\begin{array}{l}\text { Atopic dermatitis at } 2 \mathrm{y} \text { : No significant } \\
\text { associations }\end{array}$ \\
\hline $\begin{array}{l}\text { Filipiak, } 2007 \text { (3); Prospective Cohort Study } \\
\text { (German Infant Nutritional Intervention } \\
\text { Program); Germany; N: } 4753\end{array}$ & $\begin{array}{l}\text { Age of dairy product introduction: } 1-4,5-6 \text {, } \\
\text { vs. } 7-12 \text { mo }\end{array}$ & $\begin{array}{l}\text { Atopic dermatitis at } 4 \mathrm{y} \text { : No significant } \\
\text { associations }\end{array}$ \\
\hline $\begin{array}{l}\text { Goksor, } 2013 \text { (32); Prospective Cohort Study } \\
\text { (Infants of Western Sweden); Sweden; N: } \\
4051\end{array}$ & $\begin{array}{l}\text { Type of spread: Margarine, or no spread vs. } \\
\text { butter at } 1 y\end{array}$ & $\begin{array}{l}\text { Asthma at } 8 y: \mathrm{OR}=1.7,95 \% \mathrm{Cl}: 1.2,2.3 \\
\mathrm{OR}=1.2,95 \% \mathrm{Cl}: 0.7,2.3 \text {; both } \mathrm{P}<0.01\end{array}$ \\
\hline $\begin{array}{l}\text { Grimshaw, } 2013 \text { (10); Nested Case-Control } \\
\text { Study (Prevalence of Infant Food Allergy } \\
\text { EuroPrevall); UK; Food Allergy Cases: 41, } \\
\text { Controls: } 82\end{array}$ & $\begin{array}{l}\text { Age of cow's milk (as an ingredient) } \\
\text { introduction: Median in wk for cases vs. } \\
\text { controls }\end{array}$ & $\begin{array}{l}\text { Food allergy at } 2 y, \text { cases vs. controls: } 22 w k \text { vs. } \\
26 w k, P=0.049\end{array}$ \\
\hline \multirow{2}{*}{$\begin{array}{l}\text { Nwaru, } 2013 \text { (26); Prospective Cohort Study } \\
\text { (Study of Eczema and Asthma To Observe } \\
\text { the Influence of Nutrition); UK; N: } 934\end{array}$} & $\begin{array}{l}\text { Age of milk product introduction: Categorized } \\
\text { by tertiles, based on month of introduction }\end{array}$ & $\begin{array}{l}\text { Atopic dermatitis at 10y: No significant } \\
\text { associations }\end{array}$ \\
\hline & & $\begin{array}{l}\text { Allergic rhinitis at 10y: No significant } \\
\text { associations }\end{array}$ \\
\hline $\begin{array}{l}\text { Perkin, } 2016 \text { (2); Randomized Controlled } \\
\text { Trial (Enquiring about Tolerance Trial (EAT); }\end{array}$ & $\begin{array}{l}\text { Early introduction group: Introduction to } \\
\text { allergenic foods at 3mo (cow's milk (yogurt) } \\
\text { first, then (in random order) peanut, cooked }\end{array}$ & $\begin{array}{l}\text { Food allergy from 1-3y, intention to treat } \\
\text { analyses: No significant group differences based } \\
\text { on intention to treat analyses }\end{array}$ \\
\hline
\end{tabular}




\section{Reference; Study design (Study or cohort name); Country; Sample size \\ UK; Standard Introduction Group: 595, Early Introduction Group: 567}

\section{Intervention or exposure}

(boiled) hen's egg, sesame, and whitefish, and wheat last)

Standard introduction group: Introduction to allergenic foods at $6 \mathrm{mo}$ according to parenta discretion

Age of yogurt, other milk products, margarine, and butter introduction: 3-12 vs. $>12 \mathrm{mo}$

\section{Results} (Protection Against Allergy in Rural

Environments Study); Austria, Finland,

France, Germany, Switzerland; N: 1041
Roduit, 2014 (5); Prospective Cohort Study

(Protection Against Allergy in Rural

Environments Study); Austria, Finland,

France, Germany, Switzerland; N: 856
Age of yogurt, other milk products, margarine, and butter introduction: $<12 \mathrm{mo}$ vs. Never
Food allergy from 1-3y, per protocol analyses:

$2.4 \%$ vs. $6.4 \% ; P=0.03$

Milk allergy from 1-3y, per protocol analyses: No significant group differences

Atopic dermatitis at 1y: OR for yogurt $=0.41$,

Atopic dermatitis at 1y: No significant associations with age of other milk product, margarine, and butter introduction

Food allergy at 6y: No significant associations 95\% Cl: $0.23,0.73 ; \mathrm{P}<0.05$

Asthma at 6y: No significant associations

Sariachvili, 2010 (30); Nested Case-Control Study (Influence of Perinatal Factors on the Occurrence of Asthma and Allergy Study); Belgium; Atopic dermatitis cases: 252,

Controls: 305

Turati, 2016 (13); Case-Control Study; Italy; Atopic Dermatitis Cases: 451, Controls: 451
Age of dairy product or cheese introduction: $<4$ vs. $>4 \mathrm{mo}$
Atopic dermatitis cases and controls (0-4y): No significant associations
Virtanen, 2010 (28); Prospective Cohort Study); Finland; N: 1288
Study (Diabetes Prediction and Prevention
Age of dairy product (cheese, and other dairies) introduction: $<4$ vs. $>4 \mathrm{mo}$

Age of milk product introduction: Categorized by tertiles, based on month of introduction associations
Atopic dermatitis at 24mo: No significant

\section{Asthma at 5y: No significant associations}




\begin{tabular}{lll}
\hline \hline $\begin{array}{l}\text { Reference; Study design (Study or cohort } \\
\text { name); Country; Sample size }\end{array}$ & Intervention or exposure & Results \\
\hline $\begin{array}{l}\text { Yu, 2011 (14); Case-Control Study; US; } \\
\begin{array}{l}\text { Allergic Cases: 67, Non-Allergic Controls: } \\
191\end{array}\end{array}$ & $\begin{array}{l}\text { Age of dairy introduction: 0-12, 13-24, 25-36, } \\
\text { vs. >36mo }\end{array}$ & $\begin{array}{l}\text { Food allergy at 14-18y: OR=0.32, 95\% Cl: 0.18, } \\
0.57 ; \mathrm{P}<0.01\end{array}$ \\
\hline $\begin{array}{l}\text { Zutavern, 2004 (29); Prospective Cohort } \\
\text { Study; UK; N: 552 }\end{array}$ & Age of milk product introduction: <6 vs. >6mo & $\begin{array}{l}\text { Atopic dermatitis at 5y: No significant } \\
\text { associations }\end{array}$ \\
\hline $\begin{array}{l}\text { Zutavern, 2006 (8); Prospective Cohort Study } \\
\text { (Lifestyle-Related Factors on the Immune }\end{array}$ & $\begin{array}{l}\text { Age of dairy product introduction: 0-4, 5-6, } \\
\text { System and the Development of Allergies in } \\
\text { Childhood Study); Germany; N: } 2612\end{array}$ & $\begin{array}{l}\text { Atopic dermatitis at 2y: No significant } \\
\text { associations }\end{array}$ \\
\hline
\end{tabular}


Table 7. Results of studies that examined the relationship between wheat consumption during the complementary feeding period and risk of atopic disease.

\begin{tabular}{|c|c|c|}
\hline $\begin{array}{l}\text { Reference; Study design (Study or } \\
\text { cohort name); Country; Sample size }\end{array}$ & Intervention or exposure & Results \\
\hline \multirow{2}{*}{$\begin{array}{l}\text { Fergusson, } 1981 \text { (22); Prospective Cohort } \\
\text { Study (Christchurch Health and } \\
\text { Development Study); New Zealand; N: } 1156\end{array}$} & $\begin{array}{l}\text { Consumption of cereal: Presence vs. absence } \\
\text { at } 4 \mathrm{mo}\end{array}$ & $\begin{array}{l}\text { Atopic dermatitis at 2y: No significant } \\
\text { associations }\end{array}$ \\
\hline & $\begin{array}{l}\text { Amount of cereals consumed: Teaspoonful/d at } \\
4 \mathrm{mo}\end{array}$ & $\begin{array}{l}\text { Atopic dermatitis at } 2 \mathrm{y} \text { : No significant } \\
\text { associations }\end{array}$ \\
\hline $\begin{array}{l}\text { Filipiak, } 2007 \text { (3); Prospective Cohort Study } \\
\text { (German Infant Nutritional Intervention } \\
\text { Program); Germany; N: } 4753\end{array}$ & Age of cereal introduction: $1-4,5-6$, vs. $7-12$ mo & $\begin{array}{l}\text { Atopic dermatitis at 4y: No significant } \\
\text { associations }\end{array}$ \\
\hline $\begin{array}{l}\text { Grimshaw, } 2013 \text { (10); Nested Case-Control } \\
\text { Study (Prevalence of Infant Food Allergy } \\
\text { EuroPrevall); UK; Food Allergy Cases: 41, } \\
\text { Controls: } 82\end{array}$ & Age of wheat introduction: Median in wk & Food allergy at 2y: No significant associations \\
\hline $\begin{array}{l}\text { Grimshaw, } 2015 \text { (9); Nested Case-Control } \\
\text { Study (Prevalence of Infant Food Allergy } \\
\text { EuroPrevall); UK; Food Allergy Cases: } 41 \\
\text { (21 lgE-mediated), Controls: } 82\end{array}$ & Age of wheat introduction: Median in wk & $\begin{array}{l}\text { Food allergy (IgE- and non-IgE-mediated): No } \\
\text { significant associations }\end{array}$ \\
\hline $\begin{array}{l}\text { Kumar, } 2010 \text { (11); Nested Case-Control } \\
\text { Study; US; Food Allergy Cases: 411, } \\
\text { Asymptomatic Sensitized Cases: } 171 \text {, } \\
\text { Controls: } 378\end{array}$ & $\begin{array}{l}\text { Age of rice/wheat cereal introduction: }>6 \text { vs. } \\
<6 \text { mo }\end{array}$ & $\begin{array}{l}\text { Food allergy at } 6-7 y: 0 R=0.6,95 \% \mathrm{Cl}: 0.4, \\
0.996 ; \mathrm{P}<0.05\end{array}$ \\
\hline \multirow[t]{3}{*}{$\begin{array}{l}\text { Perkin, } 2016 \text { (2); Randomized Controlled } \\
\text { Trial (Enquiring about Tolerance Trial (EAT); } \\
\text { UK; Standard Introduction Group: 595, Early } \\
\text { Introduction Group: } 567\end{array}$} & $\begin{array}{l}\text { Early introduction group: Introduction to } \\
\text { allergenic foods at 3mo (cow's milk (yogurt) } \\
\text { first, then (in random order) peanut, cooked } \\
\text { (boiled) hen's egg, sesame, and whitefish, and } \\
\text { wheat last) }\end{array}$ & $\begin{array}{l}\text { Food allergy from 1-3y, intention to treat } \\
\text { analyses: No significant group differences } \\
\text { based on intention to treat analyses }\end{array}$ \\
\hline & $\begin{array}{l}\text { Standard introduction group: Introduction to } \\
\text { allergenic foods at } 6 \mathrm{mo} \text { according to parental } \\
\text { discretion }\end{array}$ & $\begin{array}{l}\text { Food allergy from } 1-3 y \text {, per protocol analyses: } \\
2.4 \% \text { vs. } 6.4 \% ; P=0.03\end{array}$ \\
\hline & & $\begin{array}{l}\text { Wheat allergy from 1-3y, per protocol analyses: } \\
\text { No significant group differences }\end{array}$ \\
\hline
\end{tabular}




\section{Reference; Study design (Study or cohort name); Country; Sample size}

Niinivirta, 2014 (25); Prospective Cohort Study; Finland; N: 223 at $6 \mathrm{mo} ; 211$ at 12mo; 185 at $24 \mathrm{mo} ; 129$ at $48 \mathrm{mo}$

\section{Intervention or exposure \\ Results}

Age of cereal introduction: 6 vs. $\geq 7 \mathrm{mo}$

Atopic dermatitis at 4y: No significant associations

Atopic dermatitis at 5y: No significant associations

(Finnish Type 1 Diabetes Prediction and $\quad>5.5 \mathrm{mo}$

Prevention Study); Finland; N: 3781
Asthma at 5y: Reduced risk for $<5,5-5.5$ vs. $>5.5 \mathrm{mo}$ (data not shown); $\mathrm{P}<0.01$

Allergic rhinitis at 5y: Reduced risk for $<5,5-5.5$ vs. $>5.5 \mathrm{mo}$ (data not shown); $\mathrm{P}<0.01$

Age of biscuits/bread introduction: $\geq 6$ vs. $<6 \mathrm{mo}$ Atopic dermatitis at 10y, all subjects: No significant associations

Atopic dermatitis at $10 \mathrm{y}$, children without atopic dermatitis by $6 \mathrm{mo}$ : $\mathrm{OR}=1.51,95 \% \mathrm{Cl}: 1.14$, $2.01 ; P=0.005$

Atopic dermatitis at $10 \mathrm{y}$, children without family atopy history: $\mathrm{OR}=1.58,95 \% \mathrm{Cl}: 1.01,2.46$; $P=0.045$

Allergic rhinitis at 10y: No significant associations

\begin{tabular}{|c|c|c|}
\hline $\begin{array}{l}\text { Poole, } 2006 \text { (36); Prospective Cohort Study } \\
\text { (Diabetes Autoimmunity Study in the } \\
\text { Young); US; N: } 1612\end{array}$ & $\begin{array}{l}\text { Age of cereal grains (wheat, barley, rye oats) } \\
\text { introduction: }>7 \text { vs. } 0-6 \text { mo }\end{array}$ & $\begin{array}{l}\text { Wheat allergy at } 4 \mathrm{y}: \mathrm{OR}=3.8,95 \% \mathrm{Cl}: 1.18 \text {, } \\
12.28 ; \mathrm{P}=0.025\end{array}$ \\
\hline \multirow{3}{*}{$\begin{array}{l}\text { Roduit, } 2012 \text { (6); Prospective Cohort Study } \\
\text { (Protection Against Allergy in Rural } \\
\text { Environments Study); Austria, Finland, } \\
\text { France, Germany, Switzerland; N: } 1041\end{array}$} & Age of cereal introduction: $3-12$ vs. $>12 \mathrm{mo}$ & $\begin{array}{l}\text { Atopic dermatitis at } 4 y \text { : No significant } \\
\text { associations }\end{array}$ \\
\hline & Age of bread introduction: $3-12$ vs. $>12 \mathrm{mo}$ & $\begin{array}{l}\text { Atopic dermatitis at } 4 y \text { : No significant } \\
\text { associations }\end{array}$ \\
\hline & Age of cake introduction: $3-12$ vs. $>12 \mathrm{mo}$ & $\begin{array}{l}\text { Atopic dermatitis at 4y: No significant } \\
\text { associations }\end{array}$ \\
\hline
\end{tabular}




\section{Reference; Study design (Study or cohort name); Country; Sample size}

Roduit, 2014 (5); Prospective Cohort Study

Environments Study); Austria, Finland,

France, Germany, Switzerland; N: 856 (Protection Against Allergy in Rural

Intervention or exposure $\quad$ Results

Age of cereal introduction: $<12 \mathrm{mo}$ vs. Never

Food allergy at 6y: No significant associations

Asthma at 6y: No significant associations

Age of bread introduction: 3-12 vs. $>12 \mathrm{mo}$

Food allergy at 6y: No significant associations

Asthma at 6y: No significant associations

Age of cake introduction: $3-12$ vs. $>12 \mathrm{mo}$

Food allergy at $6 \mathrm{y}: \mathrm{OR}=0.31,95 \% \mathrm{Cl}: 0.15$, $0.64 ; \mathrm{P}<0.05$

Asthma at 6y: No significant associations

Sariachvili, 2010 (30); Nested Case-Control Study (Influence of Perinatal Factors on the Occurrence of Asthma and Allergies Study);

Age of cereal introduction: $<4$ vs. $>4 \mathrm{mo}$ Atopic dermatitis at 0-4y: No significant associations

Belgium; Atopic dermatitis cases: 252,

Controls: 305

Tromp, 2011 (7); Prospective Cohort Study (Generation R Study); The Netherlands; N: 6905

Venter, 2016 (12); Nested Case-Control Study (Food Allergy and Intolerance Research Cohort); UK; Food Allergy Cases:

39. Controls: 78

Virtanen, 2010 (28); Prospective Cohort

Study (Diabetes Prediction and Prevention

Study); Finland; N: 1288

Yu, 2011 (14); Case-Control Study; US; Allergic Cases: 67, Non-Allergic Controls: 191
24, 25-36, >36mo
Age of wheat introduction: Categorized by

Asthma at 5y: No significant associations

tertiles, based on month of introduction

Allergic rhinitis at 5y: No significant

associations Age of wheat/gluten introduction: 0-12 vs. 13-

Atopic dermatitis at 2, 3, and 4y: No significant associations

Age of wheat introduction: <3, 3-6, 6-9, vs. 9- Food allergy at 1y: No significant associations $12 \mathrm{mo}$

Food allergy at $14-18 \mathrm{y}: \mathrm{OR}=0.18,95 \% \mathrm{Cl}$ : $0.10,0.33 ; P<0.01$ 


\begin{tabular}{|c|c|c|}
\hline $\begin{array}{l}\text { Reference; Study design (Study or } \\
\text { cohort name); Country; Sample size }\end{array}$ & Intervention or exposure & Results \\
\hline $\begin{array}{l}\text { Zutavern, } 2004 \text { (29); Prospective Cohort } \\
\text { Study; UK; N: } 552\end{array}$ & Age of cereal introduction: $<4 \mathrm{vs} .>4 \mathrm{mo}$ & $\begin{array}{l}\text { Atopic dermatitis at 5y: No significant } \\
\text { associations }\end{array}$ \\
\hline $\begin{array}{l}\text { Zutavern, } 2006 \text { (8); Prospective Cohort } \\
\text { Study (Lifestyle-Related Factors on the } \\
\text { Immune System and the Development of } \\
\text { Allergies in Childhood Study); Germany; N: } \\
2612\end{array}$ & Age of cereal introduction: $0-4,5-6$, vs. $>6 \mathrm{mo}$ & $\begin{array}{l}\text { Atopic dermatitis at 2y: No significant } \\
\text { associations }\end{array}$ \\
\hline
\end{tabular}


Table 8. Results of studies that examined the relationship between soybean consumption during the complementary feeding period and risk of atopic disease.

\begin{tabular}{|c|c|c|}
\hline $\begin{array}{l}\text { Reference; Study design (Study or } \\
\text { cohort name); Country; Sample size }\end{array}$ & Intervention or exposure & Results \\
\hline $\begin{array}{l}\text { Roduit, } 2012 \text { (6); Prospective Cohort Study } \\
\text { (Protection Against Allergy in Rural } \\
\text { Environments Study); Austria, Finland, } \\
\text { France, Germany, Switzerland N: } 1041\end{array}$ & Age of soy introduction: $3-12$ vs. $>12 \mathrm{mo}$ & $\begin{array}{l}\text { Atopic dermatitis at 4y: No significant } \\
\text { associations }\end{array}$ \\
\hline \multirow[t]{2}{*}{$\begin{array}{l}\text { Roduit, } 2014 \text { (5); Prospective Cohort Study } \\
\text { (Protection Against Allergy in Rural } \\
\text { Environments Study); Austria, Finland, } \\
\text { France, Germany, Switzerland ;N: } 856\end{array}$} & Age of soy introduction: $<12 \mathrm{mo}$ vs. Never & Food allergy at 6y: No significant associations \\
\hline & & Asthma at 6y: No significant associations \\
\hline $\begin{array}{l}\text { Tromp, } 2011 \text { (7); Prospective Cohort Study } \\
\text { (Generation R Study); The Netherlands; N: } \\
6905\end{array}$ & Age of soy introduction: Not defined & $\begin{array}{l}\text { Atopic dermatitis at 2, 3,4 and } 4 \mathrm{y} \text { : No significant } \\
\text { associations }\end{array}$ \\
\hline $\begin{array}{l}\text { Zutavern, } 2006 \text { (8); Prospective Cohort } \\
\text { Study (Lifestyle-Related Factors on the } \\
\text { Immune System and the Development of } \\
\text { Allergies in Childhood Study); Germany; N: } \\
2612\end{array}$ & $\begin{array}{l}\text { Age of soybean/nut/cacao/chocolate } \\
\text { introduction: } 0-4,5-6 \text {, vs. }>6 \text { mo }\end{array}$ & $\begin{array}{l}\text { Atopic dermatitis at 2y: No significant } \\
\text { associations }\end{array}$ \\
\hline
\end{tabular}


Table 9. Results of studies that examined the relationship between consumption of complementary foods and beverages not considered to be major allergens and risk of atopic disease.

\begin{tabular}{|c|c|c|}
\hline $\begin{array}{l}\text { Reference; Study design (Study or } \\
\text { cohort name); Country; Sample size }\end{array}$ & Intervention or exposure & Results \\
\hline \multirow{3}{*}{$\begin{array}{l}\text { Andreasyan, } 2007 \text { (39); Prospective Cohort } \\
\text { Study (Tasmanian Infant Health Survey; } \\
\text { Childhood Allergy and Respiratory Health } \\
\text { Survey); Tasmania; N: } 499\end{array}$} & $\begin{array}{l}\text { Age of fruit syrup, orange juice, water, } \\
\text { vitamins, and honey introduction: }<5 \text { vs. }>5 \text { wk }\end{array}$ & $\begin{array}{l}\text { Atopic dermatitis at } 8 y \text { : No significant } \\
\text { associations }\end{array}$ \\
\hline & & Asthma at 8y: No significant associations \\
\hline & & Allergic rhinitis at 8y: No significant associations \\
\hline \multirow{6}{*}{$\begin{array}{l}\text { Fergusson, } 1981 \text { (22); Prospective Cohort } \\
\text { Study (Christchurch Health and } \\
\text { Development Study); New Zealand; N: } \\
1156\end{array}$} & $\begin{array}{l}\text { Consumption of fruit: Presence vs. absence at } \\
4 \mathrm{mo}\end{array}$ & $\begin{array}{l}\text { Atopic dermatitis at } 2 \mathrm{y} \text { : No significant } \\
\text { associations }\end{array}$ \\
\hline & $\begin{array}{l}\text { Amount of fruit consumed: Teaspoonful (Tsp)/d } \\
\text { at } 4 \mathrm{mo}\end{array}$ & $\begin{array}{l}\text { Atopic dermatitis at } 2 \mathrm{y} \text { : No significant } \\
\text { associations }\end{array}$ \\
\hline & $\begin{array}{l}\text { Consumption of vegetables: Presence vs. } \\
\text { absence at } 4 \mathrm{mo}\end{array}$ & $\begin{array}{l}\text { Atopic dermatitis at 2y: No significant } \\
\text { associations }\end{array}$ \\
\hline & Amount of vegetables consumed: Tsp/d at $4 \mathrm{mo}$ & $\begin{array}{l}\text { Atopic dermatitis at } 2 \mathrm{y} \text { : No significant } \\
\text { associations }\end{array}$ \\
\hline & $\begin{array}{l}\text { Consumption of meat: Presence vs. absence } \\
\text { at } 4 \mathrm{mo}\end{array}$ & $\begin{array}{l}\text { Atopic dermatitis at } 2 \mathrm{y} \text { : No significant } \\
\text { associations }\end{array}$ \\
\hline & Amount of meat consumed: Tsp/d at $4 \mathrm{mo}$ & $\begin{array}{l}\text { Atopic dermatitis at } 2 \mathrm{y} \text { : No significant } \\
\text { associations }\end{array}$ \\
\hline \multirow{3}{*}{$\begin{array}{l}\text { Filipiak, } 2007 \text { (3); Prospective Cohort } \\
\text { Study (German Infant Nutritional } \\
\text { Intervention Program); Germany; N: } 4753\end{array}$} & Age of fruit introduction: $>6$ vs. $<6 \mathrm{mo}$ & $\begin{array}{l}\text { Atopic dermatitis at 4y: No significant } \\
\text { associations }\end{array}$ \\
\hline & Age of vegetable introduction: $>6$ vs. $<6 \mathrm{mo}$ & $\begin{array}{l}\text { Atopic dermatitis at } 2 \mathrm{y} \text { : No significant } \\
\text { associations }\end{array}$ \\
\hline & Age of meat introduction: $>6$ vs. $<6 \mathrm{mo}$ & $\begin{array}{l}\text { Atopic dermatitis at } 2 \mathrm{y} \text {, intervention subjects: } \\
\mathrm{OR}=1.57,95 \% \mathrm{Cl}: 0.82,2.95\end{array}$ \\
\hline
\end{tabular}




\begin{tabular}{|c|c|c|}
\hline $\begin{array}{l}\text { Reference; Study design (Study or } \\
\text { cohort name); Country; Sample size }\end{array}$ & Intervention or exposure & Results \\
\hline & & $\begin{array}{l}\text { Atopic dermatitis at } 2 \mathrm{y} \text {, intervention subjects: } \\
\mathrm{OR}=1.42,95 \% \mathrm{Cl}: 1.11,1.81\end{array}$ \\
\hline $\begin{array}{l}\text { Goksor, } 2013 \text { (32); Prospective Cohort } \\
\text { Study (Infants of Western Sweden); } \\
\text { Sweden; N: } 4051\end{array}$ & $\begin{array}{l}\text { Frequency of fermented food consumption: } 1+ \\
\text { vs. } 0 \text { time/mo at } 1 \mathrm{y}\end{array}$ & Asthma at $8 \mathrm{y}: \mathrm{OR}=0.5,95 \% \mathrm{Cl}: 0.3,0.7 ; \mathrm{P}<0.10$ \\
\hline $\begin{array}{l}\text { Harris, } 2001 \text { (38); Prospective Cohort } \\
\text { Study; UK; N: } 624\end{array}$ & Age of rice introduction: $<12 \mathrm{vs} .>12 \mathrm{mo}$ & $\begin{array}{l}\text { Atopic dermatitis (visible) at } 2 \mathrm{y}: 12 \% \text { vs. } 2 \% \text {; } \\
\mathrm{P}=0.02\end{array}$ \\
\hline \multirow{2}{*}{$\begin{array}{l}\text { Hesselmar, } 2010 \text { (24); Prospective Cohort } \\
\text { Study (ALLERGY-FLORA); Sweden; N: } 184\end{array}$} & Age of fruit introduction: Months, continuous & Food allergy at $18 \mathrm{mo}$ : No significant associations \\
\hline & & $\begin{array}{l}\text { Atopic dermatitis at } 18 \mathrm{mo} \text { : No significant } \\
\text { associations }\end{array}$ \\
\hline \multirow{3}{*}{$\begin{array}{l}\text { Niinivirta, } 2014 \text { (25); Prospective Cohort } \\
\text { Study; Finland; N: } 223 \text { at } 6 \mathrm{mo} ; 211 \text { at } \\
12 \mathrm{mo} ; 185 \text { at } 24 \mathrm{mo} ; 129 \text { at } 48 \mathrm{mo}\end{array}$} & $\begin{array}{l}\text { Age of fruit/berry introduction: }<4,4-6, \text { vs. } \\
\geq 7 \mathrm{mo}\end{array}$ & $\begin{array}{l}\text { Atopic dermatitis at 4y: No significant } \\
\text { associations }\end{array}$ \\
\hline & Age of vegetable introduction: $4 \mathrm{vs} . \geq 4 \mathrm{mo}$ & $\begin{array}{l}\text { Atopic dermatitis at } 4 \mathrm{y} \text { : No significant } \\
\text { associations }\end{array}$ \\
\hline & $\begin{array}{l}\text { Age of meat (red/white) introduction: } 6 \mathrm{vs.} \\
\geq 7 \mathrm{mo}\end{array}$ & $\begin{array}{l}\text { Atopic dermatitis at 4y: No significant } \\
\text { associations }\end{array}$ \\
\hline \multirow{6}{*}{$\begin{array}{l}\text { Nwaru, } 2013 \text { (27); Prospective Cohort } \\
\text { Study (Finnish Type } 1 \text { Diabetes Prediction } \\
\text { and Prevention Study); Finland; N: } 3781\end{array}$} & $\begin{array}{l}\text { Age of rye, oats, and barley introduction: }<5,5- \\
5.5 \text { vs. }>5.5 \mathrm{mo}\end{array}$ & $\begin{array}{l}\text { Atopic dermatitis at 5y: No significant } \\
\text { associations }\end{array}$ \\
\hline & & $\begin{array}{l}\text { Asthma at } 5 y \text { : Reduced risk with earlier } \\
\text { introduction (data not shown); } P<0.01\end{array}$ \\
\hline & & $\begin{array}{l}\text { Allergic rhinitis at 5y: Reduced risk with earlier } \\
\text { introduction (data not shown); } P<0.001\end{array}$ \\
\hline & $\begin{array}{l}\text { Age of maize, rice, millet, and buckwheat } \\
\text { introduction: }<4.5,4.5-5.5 \text { vs. }>5.5 \mathrm{mo}\end{array}$ & $\begin{array}{l}\text { Atopic Atopic dermatitis at } 5 \mathrm{y} \text { : Increased risk with } \\
\text { earlier introduction (data not shown); } \mathrm{P}<0.01\end{array}$ \\
\hline & & Asthma at 5y: No significant associations \\
\hline & & Allergic rhinitis at $5 y$ : No significant associations \\
\hline
\end{tabular}




\begin{tabular}{|c|c|c|}
\hline \multirow[t]{11}{*}{$\begin{array}{l}\text { Reference; Study design (Study or } \\
\text { cohort name); Country; Sample size }\end{array}$} & Intervention or exposure & Results \\
\hline & $\begin{array}{l}\text { Age of fruit/berry introduction: Categorized by } \\
\text { tertiles, based on month of introduction }\end{array}$ & $\begin{array}{l}\text { Atopic dermatitis at } 5 y \text { : No significant } \\
\text { associations }\end{array}$ \\
\hline & & Asthma at 5y: No significant associations \\
\hline & & Allergic rhinitis at $5 \mathrm{y}$ : No significant associations \\
\hline & $\begin{array}{l}\text { Age of root (potatoes, carrot, and turnip) } \\
\text { introduction: Categorized by tertiles, based on } \\
\text { month of introduction }\end{array}$ & $\begin{array}{l}\text { Atopic dermatitis at 5y: No significant } \\
\text { associations }\end{array}$ \\
\hline & & Asthma at 5y: No significant associations \\
\hline & & Allergic rhinitis at 5y: No significant associations \\
\hline & & Allergic rhinitis at 5y: No significant associations \\
\hline & $\begin{array}{l}\text { Age of meat introduction: Categorized by } \\
\text { tertiles, based on month of introduction }\end{array}$ & $\begin{array}{l}\text { Atopic dermatitis at } 5 y \text { : No significant } \\
\text { associations }\end{array}$ \\
\hline & & Asthma at 5y: No significant associations \\
\hline & & Allergic rhinitis at 5y: No significant associations \\
\hline \multirow{6}{*}{$\begin{array}{l}\text { Nwaru, } 2013 \text { (26); Prospective Cohort } \\
\text { Study (Study of Eczema and Asthma To } \\
\text { Observe the Influence of Nutrition); UK; N: } \\
934\end{array}$} & $\begin{array}{l}\text { Age of rice/cereal introduction: Categorized by } \\
\text { tertiles, based on month of introduction }\end{array}$ & $\begin{array}{l}\text { Atopic dermatitis at 10y: No significant } \\
\text { associations }\end{array}$ \\
\hline & & $\begin{array}{l}\text { Allergic rhinitis at } 10 y \text { : No significant } \\
\text { associations }\end{array}$ \\
\hline & $\begin{array}{l}\text { Age of fruit juice and fruit introduction: } \\
\text { Categorized by tertiles, based on month of } \\
\text { introduction }\end{array}$ & $\begin{array}{l}\text { Atopic dermatitis at 10y: : No significant } \\
\text { associations }\end{array}$ \\
\hline & & $\begin{array}{l}\text { Allergic rhinitis at } 10 y \text { : No significant } \\
\text { associations }\end{array}$ \\
\hline & $\begin{array}{l}\text { Age of vegetable introduction: Categorized by } \\
\text { tertiles, based on month of introduction }\end{array}$ & $\begin{array}{l}\text { Atopic dermatitis at 10y: : No significant } \\
\text { associations }\end{array}$ \\
\hline & & $\begin{array}{l}\text { Allergic rhinitis at } 10 y \text { : No significant } \\
\text { associations }\end{array}$ \\
\hline
\end{tabular}




\begin{tabular}{|c|c|c|}
\hline $\begin{array}{l}\text { Reference; Study design (Study or } \\
\text { cohort name); Country; Sample size }\end{array}$ & Intervention or exposure & Results \\
\hline & $\begin{array}{l}\text { Age of meat introduction: Categorized by } \\
\text { tertiles, based on month of introduction }\end{array}$ & $\begin{array}{l}\text { Atopic dermatitis at 10y: : No significant } \\
\text { associations }\end{array}$ \\
\hline & & $\begin{array}{l}\text { Allergic rhinitis at } 10 y \text { : No significant } \\
\text { associations }\end{array}$ \\
\hline \multirow{2}{*}{$\begin{array}{l}\text { Oien, } 2010 \text { (35); Prospective Cohort Study } \\
\text { (Prevention of Allergy Among Children in } \\
\text { Trondheim Study); Norway; N: } 3086\end{array}$} & $\begin{array}{l}\text { Frequency of vegetable consumption: } 1 \\
\text { time/wk or less, } 2-5 \text { times/wk, almost daily }\end{array}$ & $\begin{array}{l}\text { Atopic dermatitis at 2y: No significant } \\
\text { associations }\end{array}$ \\
\hline & & Asthma at 2y: No significant associations \\
\hline $\begin{array}{l}\text { Poole, } 2006 \text { (36); Prospective Cohort Study } \\
\text { (Diabetes Autoimmunity Study in the } \\
\text { Young); US: N: } 1612\end{array}$ & Age of rice cereal introduction: $0-6 \mathrm{vs} .>7 \mathrm{mo}$ & Food allergy at 4y: No significant associations \\
\hline \multirow{3}{*}{$\begin{array}{l}\text { Roduit, } 2012 \text { (6); Prospective Cohort Study } \\
\text { (Protection Against Allergy in Rural } \\
\text { Environments Study); Austria, Finland, } \\
\text { France, Germany, Switzerland; N: } 1041\end{array}$} & $\begin{array}{l}\text { Age of fruit and vegetable introduction: } 3-12 \text { vs. } \\
>12 \mathrm{mo}\end{array}$ & $\begin{array}{l}\text { Atopic dermatitis at } 4 \mathrm{y} \text { : No significant } \\
\text { associations }\end{array}$ \\
\hline & Age of meat introduction: $3-12$ vs. $>12 \mathrm{mo}$ & $\begin{array}{l}\text { Atopic dermatitis at } 4 \mathrm{y} \text { : No significant } \\
\text { associations }\end{array}$ \\
\hline & Age of chocolate introduction: $3-12$ vs. $>12 \mathrm{mo}$ & $\begin{array}{l}\text { Atopic dermatitis at } 4 \mathrm{y}: \mathrm{OR}=0.66,95 \% \mathrm{Cl}: 0.45 \text {, } \\
0.98 ; \mathrm{P}<0.05\end{array}$ \\
\hline \multirow{5}{*}{$\begin{array}{l}\text { Roduit, } 2014 \text { (5); Prospective Cohort Study } \\
\text { (Protection Against Allergy in Rural } \\
\text { Environments Study); Austria, Finland, } \\
\text { France, Germany, Switzerland; N: } 856\end{array}$} & $\begin{array}{l}\text { Age of fruit/vegetable introduction: } 3-12 \text { vs. } \\
>12 \mathrm{mo}\end{array}$ & Food allergy at 6y: No significant associations \\
\hline & & Asthma at 6y: No significant associations \\
\hline & Age of meat introduction: $3-12$ vs. $>12 \mathrm{mo}$ & Food allergy at 6y: No significant associations \\
\hline & & $\begin{array}{l}\text { Asthma at } 6 y: O R=0.43,95 \% \mathrm{Cl}: 0.20,0.93 \\
P<0.05\end{array}$ \\
\hline & Age of chocolate introduction: $3-12$ vs. $>12 \mathrm{mo}$ & $\begin{array}{l}\text { Food allergy at } 6 y: \text { OR=0.36, 95\% Cl: } 0.19,0.70 ; \\
P<0.05\end{array}$ \\
\hline
\end{tabular}




\section{Reference; Study design (Study or} cohort name); Country; Sample size

Sariachvili, 2010 (30); Nested Case-Control Study (Influence of Perinatal Factors on the Occurrence of Asthma and Allergies Study); Belgium; Atopic dermatitis cases: 252
Controls: 305

\section{Intervention or exposure}

Age of fruit juice introduction: $<4$ vs. $>4 \mathrm{mo}$

Age of fruit introduction: $<4$ vs. $>4$ mo

Age of vegetable introduction: $<4$ vs. $>4 \mathrm{mo}$

Age of meat introduction: $<4$ vs. $>4$ mo

Age of fruit and fruit juice introduction: $<4$ vs. $>4 \mathrm{mo}$ $>4 \mathrm{mo}$

Strassburger, 2010 (37); Prospective Cohort Study; Brazil; N: 354

Age of cereal (maize/tapioca, rice, pasta, gluten-free pasta) introduction: $<4$ vs. $>4 \mathrm{mo}$

Turati, 2016 (13); Case-Control Study; Italy Atopic Dermatitis Cases: 451, Controls: 451

Age of fruit introduction: $<4$ vs. $>4 \mathrm{mo}$

Age of vegetable, legume and root

introduction: $<4$ vs. $>4 \mathrm{mo}$

Age of meat (poultry, pork, beef) introduction: $<4$ vs. $>4 \mathrm{mo}$

Virtanen, 2010 (28); Prospective Cohort

Study (Diabetes Prediction and Prevention

Study); Finland; N: 1288

vs. $>5.5 \mathrm{mo}$
Age of salty pureed food introduction: $<4$ vs.

\section{Results}

Atopic dermatitis at 0-4y: OR=0.64, 95\% Cl: 045, $0.93 ; P=0.02$

Atopic dermatitis at 0-4y: No significant associations

Atopic dermatitis at 0-4y: No significant associations

Atopic dermatitis at 0-4y: No significant associations

Asthma at 3-4y: No significant associations

Asthma at 3-4y: No significant associations

Atopic dermatitis at 24mo: No significant associations

Atopic dermatitis at 24mo: Reduced risk with earlier introduction (data not shown); $\mathrm{P}=0.02$

Atopic dermatitis at 24mo: Trend for reduced risk with earlier introduction; $P=0.06$

Age of oat introduction: $<5$ vs. $>5.5 \mathrm{mo}, 5-5.5 \quad$ Asthma at $5 \mathrm{y}$ : $\mathrm{OR}=0.31,95 \% \mathrm{Cl}: 0.10,0.97$

$\mathrm{OR}=0.39,95 \% \mathrm{Cl}: 0.21,0.72 ; \mathrm{P}=0.005$

Allergic rhinitis at 5y: No significant associations

Age of barley, rye introduction: Categorized by Asthma at 5y: No significant associations tertiles, based on month of introduction 


\begin{tabular}{|c|c|c|}
\hline \multirow[t]{12}{*}{$\begin{array}{l}\text { Reference; Study design (Study or } \\
\text { cohort name); Country; Sample size }\end{array}$} & Intervention or exposure & Results \\
\hline & & Allergic rhinitis at 5y: No significant associations \\
\hline & $\begin{array}{l}\text { Age of maize, rice, millet and buckwheat } \\
\text { introduction: Categorized by tertiles, based on } \\
\text { month of introduction }\end{array}$ & Asthma at 5y: No significant associations \\
\hline & & Allergic rhinitis at 5y: No significant associations \\
\hline & $\begin{array}{l}\text { Age of fruit/berry introduction: Categorized by } \\
\text { tertiles, based on month of introduction }\end{array}$ & Asthma at 5y: No significant associations \\
\hline & & Allergic rhinitis at $5 \mathrm{y}$ : No significant associations \\
\hline & $\begin{array}{l}\text { Age of root (potato, carrot, turnip and swede) } \\
\text { introduction: Categorized by tertiles, based on } \\
\text { month of introduction }\end{array}$ & Asthma at 5y: No significant associations \\
\hline & & Allergic rhinitis at $5 \mathrm{y}$ : No significant associations \\
\hline & $\begin{array}{l}\text { Age of cabbage (cauliflower, broccoli, kale and } \\
\text { Chinese, red and turnip cabbage) introduction: } \\
\text { Categorized by tertiles, based on month of } \\
\text { introduction }\end{array}$ & Asthma at 5y: No significant associations \\
\hline & & Allergic rhinitis at 5y: No significant associations \\
\hline & $\begin{array}{l}\text { Age of meat (pork, beef, chicken and other } \\
\text { meat) introduction: Categorized by tertiles, } \\
\text { based on month of introduction }\end{array}$ & Asthma at 5y: No significant associations \\
\hline & & Allergic rhinitis at 5y: No significant associations \\
\hline \multirow[t]{3}{*}{$\begin{array}{l}\text { Zutavern, } 2004 \text { (29); Prospective Cohort } \\
\text { Study; UK; N: } 552\end{array}$} & Age of rice introduction: $<3$ vs. $>3 \mathrm{mo}$ & $\begin{array}{l}\text { Atopic dermatitis at 5y: No significant } \\
\text { associations }\end{array}$ \\
\hline & $\begin{array}{l}\text { Age of fruit or vegetable introduction: }<4 \text { vs. } \\
>4 \mathrm{mo}\end{array}$ & $\begin{array}{l}\text { Atopic dermatitis at 5y: No significant } \\
\text { associations }\end{array}$ \\
\hline & Age of meat introduction: $<5 \mathrm{vs} .>5 \mathrm{mo}$ & $\begin{array}{l}\text { Atopic dermatitis at } 5 y \text { : No significant } \\
\text { associations }\end{array}$ \\
\hline $\begin{array}{l}\text { Zutavern, } 2006 \text { (8); Prospective Cohort } \\
\text { Study (Lifestyle-Related Factors on the }\end{array}$ & Age of fruit introduction: $>6$ vs. $0-4,5-6 \mathrm{mo}$ & $\begin{array}{l}\text { Atopic dermatitis at 2y: No significant } \\
\text { associations }\end{array}$ \\
\hline
\end{tabular}


Reference; Study design (Study or

Intervention or exposure

Results

cohort name); Country; Sample size

Immune System and the Development of

Allergies in Childhood Study); Germany; N:

2612

Age of vegetable introduction: $>6$ vs. $0-4,5$
$6 \mathrm{mo}$

Age of meat introduction: $>6$ vs. 0-4, 5-6mo

Age of soybean, nut, and cacao/chocolate

introduction: $>6$ vs. $0-4,5-6 \mathrm{mo}$
Atopic dermatitis (doctor-diagnosed) at $2 \mathrm{y}$ :

$\mathrm{OR}=1.49,95 \% \mathrm{Cl}: 1.06,2.10 ; \mathrm{P}<0.05$

Atopic dermatitis (with early skin or allergic

symptoms) at 2y: $\mathrm{OR}=1.64,95 \% \mathrm{Cl}: 1.01,2.65$; $\mathrm{P}<0.05$

Atopic dermatitis (doctor-diagnosed) at $2 \mathrm{y}$ :

$\mathrm{OR}=1.63,95 \% \mathrm{Cl}: 1.00,2.65 ; \mathrm{P}<0.05$

Atopic dermatitis at 2y: No significant associations 
Table 10. Results of studies examining the relationship between diet diversity and dietary patterns during the complementary feeding period and food allergy, atopic dermatitis, asthma, and allergic rhinitis.

\begin{tabular}{|c|c|c|}
\hline $\begin{array}{l}\text { Reference; Study Design (Study or } \\
\text { Cohort Name); Country; Sample Size }\end{array}$ & Intervention/Exposure & Results \\
\hline \multicolumn{3}{|l|}{ Diet Diversity } \\
\hline $\begin{array}{l}\text { Fergusson, } 1981 \text { (22); Prospective } \\
\text { Cohort Study (Christchurch Health and } \\
\text { Development Study); v New Zealand; } \\
\text { N: } 1156\end{array}$ & $\begin{array}{l}\text { Diet diversity at } 4 \mathrm{mo}: 0,1-2,3-4,5+\text { food } \\
\text { groups }\end{array}$ & $\begin{array}{l}\text { Atopic dermatitis at } 2 \mathrm{y}: 0 \text { food groups: } 13 \% ; 1-2 \text { food } \\
\text { groups: } 17 \% ; 3-4 \text { food groups: } 17 \% ; 5+\text { food groups: } \\
33 \% ; \mathrm{P}<0.05)\end{array}$ \\
\hline $\begin{array}{l}\text { Fergusson, } 1982 \text { (42); Prospective } \\
\text { Cohort Study (Christchurch Health and } \\
\text { Development Study); New Zealand; N: } \\
1143\end{array}$ & $\begin{array}{l}\text { Diet diversity at } 4 \mathrm{mo}: 0,1-3,4+\text { food } \\
\text { groups }\end{array}$ & $\begin{array}{l}\text { Atopic dermatitis at } 3 y: 0 \text { food groups: } 16.6 \% \text { vs. } 4+ \\
\text { food groups: } 25 \% ; P<0.01 \text { ) }\end{array}$ \\
\hline $\begin{array}{l}\text { Fergusson,1990 (40); Prospective } \\
\text { Cohort Study (Christchurch Health and } \\
\text { Development Study); New Zealand; N: } \\
1067\end{array}$ & $\begin{array}{l}\text { Diet diversity at } 4 \mathrm{mo}: 0,1-3,4+\text { food } \\
\text { groups }\end{array}$ & $\begin{array}{l}\text { Atopic dermatitis at 10y: } 0 \text { food groups: } \beta=1,1-3 \text { food } \\
\text { groups: } \beta=1.69,4+\text { food groups: } \beta=2.87 ; P<0.01\end{array}$ \\
\hline $\begin{array}{l}\text { Fergusson, } 1994 \text { (41); Prospective } \\
\text { Cohort Study (Christchurch Health and } \\
\text { Development Study); New Zealand; N: } \\
1141\end{array}$ & $\begin{array}{l}\text { Diet diversity at } 4 \mathrm{mo}: 0,1-3,4+\text { food } \\
\text { groups }\end{array}$ & $\begin{array}{l}\text { Atopic dermatitis at 10y: } 0 \text { food groups: } 4.9 \% ; 1-3 \text { food } \\
\text { groups: } 8.2 \% ; 4+\text { food groups: } 13.4 \% ; P<0.01\end{array}$ \\
\hline \multirow{2}{*}{$\begin{array}{l}\text { Filipiak, } 2007 \text { (3); Prospective Cohort } \\
\text { Study (German Infant Nutritional } \\
\text { Intervention Program); Germany; N: } \\
4753\end{array}$} & $\begin{array}{l}\text { Diet diversity at } 4 \mathrm{mo}: 0,1-2,3-8 \text { food } \\
\text { groups }\end{array}$ & $\begin{array}{l}\text { Atopic dermatitis at } 4 y \text { (doctor-diagnosed, } \\
\text { symptomatic): No significant associations }\end{array}$ \\
\hline & $\begin{array}{l}\text { Diet diversity at } 6 \mathrm{mo}: 0,1-2,3-4,5-8 \text { food } \\
\text { groups }\end{array}$ & \\
\hline \multirow{2}{*}{$\begin{array}{l}\text { Nwaru, } 2014 \text { (45); Prospective Cohort } \\
\text { Study (Finnish Type } 1 \text { Diabetes } \\
\text { Prediction and Prevention Study); } \\
\text { Finland; N: 3,142 }\end{array}$} & $\begin{array}{l}\text { Diet diversity at } 3 \mathrm{mo}: 0,1-2,2+\text { food } \\
\text { groups }\end{array}$ & $\begin{array}{l}\text { Atopic dermatitis, asthma (any, atopic, non-atopic), } \\
\text { allergic rhinitis at 5y: No significant associations }\end{array}$ \\
\hline & $\begin{array}{l}\text { Diet diversity at } 4 \mathrm{mo}: 0,1-2,3-4,4+\text { food } \\
\text { groups }\end{array}$ & $\begin{array}{l}\text { Asthma (non-atopic) at } 5 y: 1-2 \text { vs. }>4 \text { food groups at } \\
4 \mathrm{mo}: \mathrm{HR}=0.26,95 \% \mathrm{Cl}: 0.09,0,75 ; \mathrm{P}=0.03\end{array}$ \\
\hline
\end{tabular}




\begin{tabular}{|c|c|c|}
\hline $\begin{array}{l}\text { Reference; Study Design (Study or } \\
\text { Cohort Name); Country; Sample Size }\end{array}$ & Intervention/Exposure & Results \\
\hline
\end{tabular}

Atopic dermatitis/, asthma (any, atopic), allergic rhinitis at 5y: No significant associations

Diet diversity at 6mo: 0-4, 5-6, 7-8, 8+ food
groups

Asthma (atopic) at 5y: 0-4 vs. $>8$ food groups: $\mathrm{HR}=2.52,95 \% \mathrm{Cl}: 1.01,6.29 ; 5-6$ vs. $>8$ food groups: $\mathrm{HR}=2.43,95 \% \mathrm{Cl}: 1.23,4.82 ; \mathrm{P}=0.04$

Allergic rhinitis at $5 \mathrm{y}: 0-4$ vs. $>8$ food groups: $H R=2.16$, $95 \% \mathrm{Cl}: 1.20,3.89 ; 5-6$ vs. $>8$ food groups: $\mathrm{HR}=1.91$, $95 \% \mathrm{Cl}: 1.24,2.95 ; \mathrm{P}=0.02$

Atopic dermatitis, asthma (any, non-atopic) at 5y: No significant associations

\begin{tabular}{ll}
\hline Diet diversity at $12 \mathrm{mo}: 0-7,8-9,10-11,11+$ & Asthma (any) at 5y: $0-7$ vs. $>11$ food groups: $\mathrm{HR}=4.19$, \\
food groups & $95 \% \mathrm{Cl}: 2.31,7.58 ; 8-9$ vs. $>11$ food groups: $\mathrm{HR}=1.87$, \\
& $95 \% \mathrm{Cl}: 1.17,2.98 ; \mathrm{P}<0.001$
\end{tabular}
$95 \%$ Cl: $1.17,2.98 ; \mathrm{P}<0.001$

Asthma (atopic) at 5y: 0-7 vs. $>11$ food groups: $\mathrm{HR}=5.17,95 \% \mathrm{Cl}: 2.34,11.42 ; 8-9$ vs. $>11$ food groups: $\mathrm{HR}=2.93,95 \% \mathrm{Cl}: 1.60,5.37 ; \mathrm{P}<0.001$

Allergic rhinitis at $5 \mathrm{y}: 0-7 \mathrm{vs.}>11$ food groups: $\mathrm{HR}=3.10,95 \% \mathrm{Cl}: 1.66,5.78 ; 8-9$ vs. $>11$ food groups: $\mathrm{HR}=2.43,95 \% \mathrm{Cl}: 1.63,3.63 ; \mathrm{P}<0.001$

Atopic dermatitis, asthma (non-atopic) at 5y: No significant associations

\begin{abstract}
Roduit, 2012 (6); Prospective Cohort Study (Protection Against Allergy-Study in Rural Environments Study); Austria,

Finland, France, Germany, Switzerland; $\mathrm{N}: 1,041$
\end{abstract}

Diet diversity from 3-12mo: 0-3, 4-5, 6 food groups

Atopic dermatitis at 1y: 6 vs. 4-5 food groups: $\mathrm{OR}=1.72 ; 95 \% \mathrm{Cl}: 1.06,2.8, \mathrm{P}=0.03 ; 6$ vs. $0-3$ groups: $\mathrm{OR}=2.87 ; 95 \% \mathrm{Cl}: 1.26,6.56, \mathrm{P}=0.01$; Per food group introduced: $\mathrm{OR}=0.75,95 \% \mathrm{Cl}: 0.62,0.91, \mathrm{P}=0.003$

Atopic dermatitis at $1 \mathrm{y}$ with no symptoms by $6 \mathrm{mo}: 0-1$ vs. $>3$ food groups: $\mathrm{OR}=2.12,95 \% \mathrm{Cl}: 1.12,4.03$; $\mathrm{P}=0.02$

Roduit, 2014 (5); Prospective Cohort

Study (Protection Against Allergy-Study

in Rural Environments Study); Austria,
Diet diversity from 3-12mo: 0-3, 4-5, 6 food groups
Food allergy at $6 y$ : 6 vs $4-5$ food groups: OR=1.85; $95 \%$ Cl: $1.02,3.35 ; 6$ vs $0-3$ food groups: $O R=4.43$, 


\section{Reference; Study Design (Study or Cohort Name); Country; Sample Size}

Finland, France, Germany, Switzerland;

N: 856

\section{Intervention/Exposure}

Results

95\% Cl: 1.62, 12.1; Per food group introduced:

$\mathrm{OR}=0.70,95 \% \mathrm{Cl}: 0.57,0.86$; all $\mathrm{P}<0.05$

Asthma (any, atopic, non-atopic) at $6 y$ : 6 vs $0-3$ food groups: $\mathrm{OR}=3.77,95 \% \mathrm{Cl}: 1.23,11.53$; Per food group introduced: $\mathrm{OR}=0.76,95 \% \mathrm{Cl}$ : 0.60, 0.96; both $\mathrm{P}<0.05$

Allergic rhinitis at 6y: No significant associations Atopic dermatitis at 1y: No significant associations

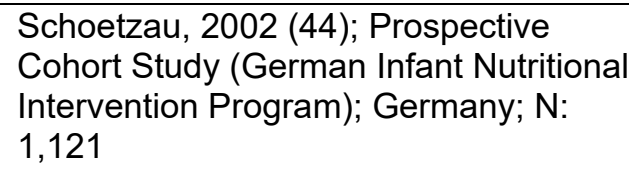

Turati, 2016 (13); Case-control study; Italy; Atopic Dermatitis Cases: 451, Controls: 451

Diet diversity at 24wk: Continuous, total

number of food groups consumed

Diet diversity at 4mo: 0, 1-2, 3-22 food groups

Diet diversity at 5mo: $0,1-7,8-22$ food groups

Zutavern, 2006 (8); Prospective Cohort
Study (Lifestyle-Related Factors on the
Immune System and the Development
of Allergies in Childhood); Germany; N:
2612

Diet diversity at 4mo: 0, 1-2, 3-8 food groups
Diet diversity at 6mo: 0, 1-2, 3-4, 5-8 food groups

Zutavern, 2008 (43); Prospective Cohort Study (Lifestyle-Related Factors on the Immune System and the Development of Allergies in Childhood); Germany; N: 2073

\section{Diet diversity at 4mo: 0, 1-2, 3-8 food}

groups

\section{Atopic dermatitis from 3-24mo: 0 vs. 8-22 food groups:} $\mathrm{OR}=0.44,95 \% \mathrm{Cl}: 0.21,0.91 ; \mathrm{P}=0.025$

\section{Atopic dermatitis from 3-24mo: 0 vs. 3-22 food groups: $\mathrm{OR}=0.30,95 \% \mathrm{Cl}: 0.11,0.81 ; \mathrm{P}=0.017$}

Atopic dermatitis at 2y: No significant associations

Atopic dermatitis (doctor-diagnosed) at 2y: 3-4 vs. 5-8 food groups, OR=0.66, 95\% Cl: 0.46, 0.94; $\mathrm{P}<0.05$

Atopic dermatitis (early skin or allergic symptoms) at 2y: 3-4 vs, 5-8 food groups: OR=0.47, 95\% Cl: 0.28 , $0.77 ; \mathrm{P}<0.05)$

Atopic dermatitis at 6y in children without early skin or allergic symptoms: 0 vs. 1-2 food groups: $O R=1.00$ $95 \% \mathrm{Cl}: 0.45,2.20 ; 0$ vs. $3-8$ food groups: $2.72,95 \%$ $\mathrm{Cl}: 1.24,5.99$

Asthma at 6y: No significant associations 


\begin{tabular}{|c|c|c|}
\hline $\begin{array}{l}\text { Reference; Study Design (Study or } \\
\text { Cohort Name); Country; Sample Size }\end{array}$ & Intervention/Exposure & Results \\
\hline
\end{tabular}

Allergic rhinitis at 6y: No significant associations

Dietary Patterns

Grimshaw, 2014 (46); Nested CaseControl Study (Prevalence of Infant Food Allergy EuroPrevall); UK; Food Allergy Cases: 41 , Controls: 82
Infant guidelines pattern: High scores for 'healthy' foods [commercial baby food, toddler snacks, carrots, potatoes,

bananas, lentils, broccoli) and low scores for adult foods (potato products, ready meals, sauces); adherence scores from 0 $12 \mathrm{mo}$

Finger food pattern: High scores for healthy finger foods and low scores for unhealthy finger foods and pureed baby food; adherence scores from 0-12mo

Adult food pattern: High scores for adult foods and low scores for 'healthy' foods; adherence scores from 0-12mo

Grimshaw, 2015 (9); Nested CaseControl Study (Prevalence of Infant Food Allergy EuroPrevall); UK; Food Allergy Cases: 41, Controls: 82

Healthy dietary pattern: High scores for 'healthy' foods (commercial baby food, toddler snacks, carrots, potatoes, bananas, lentils, broccoli) and low scores for adult foods (potato products, ready meals, sauces); adherence scores from 0 $12 \mathrm{mo}$
Food allergy at $2 \mathrm{y}: \mathrm{OR}=2.136,95 \% \mathrm{Cl}: 1.233,3.700$, $\mathrm{P}<0.05$

Food allergy at 2y: No significant associations

Food allergy at 2y: No significant associations

Food allergy at 2y: OR=0.155, 95\% Cl: $0.028,0.868$; $\mathrm{P}=0.034$ $0.16,0.66 ; \mathrm{P}=0.012$

Food allergy (non-IgE-mediated) at $2 \mathrm{y}$ : OR $=0.28,95 \%$ Cl: $0.09,0.87 ; P=0.028$ 


\section{ANALYTIC FRAMEWORK}

The analytic framework (Figure 1) illustrates the overall scope of the systematic review, including the population, the interventions and/or exposures, comparators, and outcomes of interest. It also includes definitions of key terms and identifies key confounders considered in the systematic review. The analytic framework in Figure 1 is for systematic reviews conducted to examine the relationship between complementary feeding and food allergy, atopic dermatitis/eczema, asthma, and allergic rhinitis

\section{Figure 1: Analytic framework}

\begin{tabular}{|c|c|c|c|}
\hline \multicolumn{2}{|l|}{ Target Population } & \multicolumn{2}{|l|}{ Key Definitions } \\
\hline \multicolumn{2}{|c|}{$\begin{array}{l}\text { Generally healthy infants fed human milk, infant formula, or both, } \\
\text { examined through age } 18 \text { years }\end{array}$} & \multirow{4}{*}{\multicolumn{2}{|c|}{$\begin{array}{l}\text { Complementary feeding is defined as the process that starts when } \\
\text { human milk or infant formula is complemented by other foods and } \\
\text { beverages. The complementary feeding period typically continues to } 24 \\
\text { months as the young child transitions fully to family foods. } \\
\text { Complementary foods and beverages (CFB) are food and beverages } \\
\text { (liquids, semisolids, and solids) other than human milk or infant formula } \\
\text { provided to an infant or young child to provide nutrients and energy. }\end{array}$}} \\
\hline & 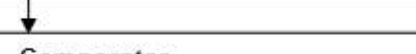 & & \\
\hline Intervention/Exposure & Comparator & & \\
\hline $\begin{array}{l}\text { - Timing of introduction of } \\
\text { complementary foods } \\
\text { and beverages (CFB) }\end{array}$ & $\begin{array}{l}\text { - Different timing of introduction } \\
\text { of CFB }\end{array}$ & & \\
\hline \multirow[t]{2}{*}{$\begin{array}{l}\text { Types and amounts of } \\
\text { CFB }\end{array}$} & $\begin{array}{l}\text { - Different types and amounts } \\
\text { of CFB }\end{array}$ & Key Confounders & Other Confounders \\
\hline & $\downarrow$ & - Education & - Maternal age \\
\hline \multicolumn{2}{|l|}{ Health Outcomes } & $\begin{array}{l}\text { - Socioeconomic status } \\
\text { - Sex }\end{array}$ & $\begin{array}{l}\text { - Maternal diet } \\
\text { - Maternal weight }\end{array}$ \\
\hline \multirow{2}{*}{\multicolumn{2}{|c|}{$\begin{array}{l}\text { - Incidence, prevalence, and/or severity of: } \\
\text { ○ Food allergy } \\
\circ \text { Atopic dermatitis/eczema } \\
\text { ○ Asthma } \\
\circ \text { Allergic rhinitis }\end{array}$}} & $\begin{array}{l}\text { - Race and/or ethnicity } \\
\text { Milk feeding practices } \\
\text { (breast milk, infant } \\
\text { formula, or both) } \\
\text { - Birth size }\end{array}$ & $\begin{array}{l}\text { - Maternal parity } \\
\text { - Childcare arrangement } \\
\text { - Marital status/head of household } \\
\text { - Medical support/access } \\
\text { - Participation in a supplemental }\end{array}$ \\
\hline & & $\begin{array}{l}\text { - Gestational age } \\
\text { - Aigarette smoke exposure } \\
\text { - Household pets }\end{array}$ & $\begin{array}{l}\text { food program } \\
\text { - Home environment } \\
\text { - Rural or urban setting } \\
\text { - Antibiotic use }\end{array}$ \\
\hline
\end{tabular}

\section{SEARCH PLAN AND RESULTS}

\section{Inclusion and exclusion criteria}

The inclusion and exclusion criteria are a set of characteristics to determine which studies will be included or excluded in the systematic review. This table provides the inclusion and exclusion criteria for the systematic review question(s): What is the relationship between timing of introduction of complementary foods/beverages or types and amounts of complementary foods/beverages and food allergy, atopic dermatitis/eczema, asthma, and allergic rhinitis?

\section{Table 11. Inclusion and exclusion criteria}

\begin{tabular}{lll}
\hline \hline Category & Inclusion Criteria & Exclusion Criteria \\
\hline Study design & Randomized controlled trials & Cross-sectional studies \\
\hline
\end{tabular}




\begin{tabular}{|c|c|c|}
\hline Category & Inclusion Criteria & Exclusion Criteria \\
\hline & Non-randomized controlled trials & Uncontrolled studies \\
\hline & Prospective cohort studies & Pre/post studies without a control \\
\hline & Retrospective cohort studies & Narrative reviews \\
\hline & Case-control studies & Systematic reviews \\
\hline & Pre/post studies with a control & Meta-analyses \\
\hline \multirow[t]{2}{*}{$\begin{array}{l}\text { Independent } \\
\text { variable } \\
\text { (intervention or } \\
\text { exposure) }\end{array}$} & $\begin{array}{l}\text { Timing of introduction to complementary } \\
\text { foods and beverages (CFB) (i.e., foods } \\
\text { and beverages (liquids, semisolids, and } \\
\text { solids) other than human milk or infant } \\
\text { formula provided to an infant or young } \\
\text { child to provide nutrients and energy) }\end{array}$ & $\begin{array}{l}\text { Consumption of fluid cow's milk before } 12 \text { months of } \\
\text { age }\end{array}$ \\
\hline & Types or amounts of CFB & \\
\hline \multirow[t]{2}{*}{ Comparator } & Different timing of introduction of CFB & \\
\hline & Different types and amounts of CFB & \\
\hline \multirow[t]{2}{*}{$\begin{array}{l}\text { Dependent } \\
\text { variables } \\
\text { (outcomes) }\end{array}$} & $\begin{array}{l}\text { Incidence, prevalence, and/or severity } \\
\text { of: food allergy, atopic } \\
\text { dermatitis/eczema, asthma, and/or } \\
\text { allergic rhinitis }\end{array}$ & $\begin{array}{l}\text { Food allergy when diagnosis was based solely on } \\
\text { food allergen sensitization (i.e., skin prick test, or } \\
\text { serum lgE measure) }\end{array}$ \\
\hline & & $\begin{array}{l}\text { Asthma when diagnosis was based solely on report } \\
\text { of wheeze }\end{array}$ \\
\hline Date range & January 1980 - February 2017 & \\
\hline Language & Studies published in English & Studies published in languages other than English \\
\hline $\begin{array}{l}\text { Publication } \\
\text { status }\end{array}$ & $\begin{array}{l}\text { Studies published in peer-reviewed } \\
\text { journals }\end{array}$ & $\begin{array}{l}\text { Grey literature, including unpublished data, } \\
\text { manuscripts, reports, abstracts, conference } \\
\text { proceedings }\end{array}$ \\
\hline Country ${ }^{1,2,3}$ & $\begin{array}{l}\text { Studies conducted in Very High or High } \\
\text { Human Development Countries }\end{array}$ & $\begin{array}{l}\text { Studies conducted in Medium or Low Human } \\
\text { Development Countries }\end{array}$ \\
\hline \multirow[t]{3}{*}{$\begin{array}{l}\text { Study } \\
\text { participants }\end{array}$} & Human subjects & $\begin{array}{l}\text { Hospitalized patients, not including birth and } \\
\text { immediate post-partum hospitalization of healthy } \\
\text { babies }\end{array}$ \\
\hline & Males & \\
\hline & Females & \\
\hline \multirow[t]{2}{*}{$\begin{array}{l}\text { Age of study } \\
\text { participants }\end{array}$} & $\begin{array}{l}\text { Age at intervention or exposure: Infants } \\
(0-12 \mathrm{mo}) \text {; Toddlers }(12-24 \mathrm{mo})\end{array}$ & $\begin{array}{l}\text { Age at intervention or exposure: Child ( } 2-5 \text { years } \\
\text { (y)); Child (6-12y); Adolescents (13-18y); Adults } \\
\text { (19y and older); Older adults ( } 65 \text { to } 79 y) ; \text { Older } \\
\text { adults }(80+y)\end{array}$ \\
\hline & $\begin{array}{l}\text { Age at outcome: Infants }(0-12 \mathrm{mo} \text {; food } \\
\text { allergy, atopic dermatitis, allergic } \\
\text { rhinitis); Toddlers ( } 12-24 \mathrm{mo} \text {; food } \\
\text { allergy, atopic dermatitis, allergic } \\
\text { rhinitis); Child ( } 2-5 \text { years (y)); Child (6- } \\
\text { 12y); Adolescents (13-18y) }\end{array}$ & $\begin{array}{l}\text { Age at outcome: Infants ( } 0-12 \text { months) (asthma); } \\
\text { Toddlers ( } 12-24 \text { months) (asthma); Adults (19y and } \\
\text { older); Older adults ( } 65 \text { to } 79 y) \text {; Older adults }(80+y)\end{array}$ \\
\hline $\begin{array}{l}\text { Health status of } \\
\text { study } \\
\text { participants }\end{array}$ & $\begin{array}{l}\text { Studies done in generally healthy } \\
\text { populations }\end{array}$ & $\begin{array}{l}\text { Studies that exclusively enroll subjects with a } \\
\text { disease or with the health outcome of interest }\end{array}$ \\
\hline
\end{tabular}




\begin{tabular}{|c|c|c|}
\hline Category & "Inclusion Criteria & Exclusion Criteria \\
\hline & $\begin{array}{l}\text { Studies done in populations where } \\
\text { infants were full term }(\geq 37 \text { wk gestational } \\
\text { age) }\end{array}$ & $\begin{array}{l}\text { Studies done in hospitalized or malnourished } \\
\text { subjects }\end{array}$ \\
\hline & $\begin{array}{l}\text { Studies done in populations with } \\
\text { elevated chronic disease risk, or that } \\
\text { enroll some participants with a disease } \\
\text { or with the health outcome of interest }\end{array}$ & $\begin{array}{l}\text { Studies of exclusively pre-term babies (gestational } \\
\text { age }<37 \mathrm{wk}) \text { or babies that are small for gestational } \\
\text { age }(<2500 \mathrm{~g})\end{array}$ \\
\hline & & $\begin{array}{l}\text { Studies of subjects with infectious diseases (e.g. } \\
\text { HIVIAIDS) (or with mothers diagnosed with an } \\
\text { infectious disease) }\end{array}$ \\
\hline \multicolumn{3}{|c|}{$\begin{array}{l}1 \text { United Nations Development Programme. Human Development Report 2014: Reducing Vulnerabilities and } \\
\text { Building Resilience. Available from: http://hdr.undp.org/en/content/human-development-report-2014. (32) }\end{array}$} \\
\hline \multicolumn{3}{|c|}{$\begin{array}{l}2 \text { Medium Development Countries were originally included, but due to concerns about generalizability to the U.S. of } \\
\text { study participants (i.e., baseline health status) and CFB typically consumed, a decision was made to exclude } \\
\text { "Medium" countries in October } 2017 \text {. }\end{array}$} \\
\hline \multicolumn{3}{|c|}{$\begin{array}{l}{ }^{3} \text { When a country was not included in the Human Development Report 2014, country classification from the World } \\
\text { Bank was used instead. (33) }\end{array}$} \\
\hline
\end{tabular}

\section{Search terms and electronic databases used}

PubMed, US National Library of Medicine (1966 to 8 February 2017):

Date(s) Searched: 2/8/2017

Search Terms:

Date range searched: $1980-2 / 8 / 17$

(Complementary OR supplementary OR wean* OR transition* OR introduc* OR "Infant Nutritional Physiological Phenomena"[Mesh:noexp] OR weaning[mesh] OR bottle*)

AND (feeding* OR food* OR beverage*[tiab] OR beverages[mh] OR eating OR

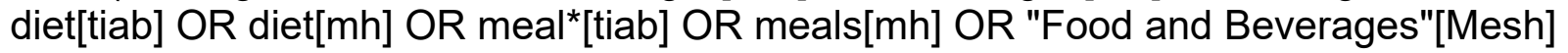
OR diets[tiab] OR cereal*[tiab] OR "Edible Grain"[Mesh] OR bread*[tiab] OR whole grain* OR juice*[tiab] OR milk[tiab] OR "Milk"[Mesh] OR dairy[tiab] OR "Dairy Products"[Mesh] OR meat[tiab] OR cheese[tiab] OR yogurt[tiab] OR yoghurt*[tiab] OR fruit*[tiab] OR "Fruit"[Mesh] OR vegetable*[tiab] OR "Vegetables"[Mesh] OR egg*[tiab] OR "Eggs"[Mesh] OR nut[tiab] OR nuts[tiab] OR peas[tiab] OR beans[tiab] OR legume*[tiab] OR snack ${ }^{*}[\mathrm{tiab}] \mathrm{OR}$ bread[mh] OR honey[mh] OR vegetable*[tiab] OR "Vegetables"[Mesh] OR egg*[tiab] OR "Eggs"[Mesh:noexp] OR "egg white"[mh] OR "egg yolk"[mh] OR snack*[tiab] OR candy[mh] OR "Fast Foods"[Mesh] OR meat[mh] OR molasses[mh] OR nuts[mh] OR "Raw Foods"[Mesh] OR seeds[mh])

OR

"infant food"[mesh] OR infant feed* OR Bottle feeding[mh] OR bottle feeding*[tiab] OR bottle feeding OR bottle-feeding*[tiab] OR bottle-feedings OR bottle-fed[tiab] OR "bottle fed"[tiab] OR solid food*

AND 
NOT (editorial[ptyp] OR comment[ptyp] OR news[ptyp] OR letter[ptyp] OR review[ptyp] OR systematic[sb])

("Study Characteristics" [Publication Type] OR "clinical trial"[ptyp] OR "Epidemiologic Studies"[Mesh] OR "Support of Research"[ptyp] OR cohort[tiab] OR observational[tiab] OR retrospective[tiab] OR longitudinal[tiab] OR trial[tiab] OR trials[tiab] OR case control*[tiab] OR case-control*[tiab] OR before-after stud ${ }^{*}[$ tiab] OR before after stud*[tiab]

(includes age filter: Filters: Infant: birth-23 months and preschool child 2-5 yrs)

OR ((Solid food*) OR solids))

AND

(("Allergy and Immunology"[Mesh:NoExp] OR allergy[tiab] OR allergies[tiab] OR allergic[tiab] OR Hypersensitivit*[tiab]) AND (food OR peanut* OR nut OR nuts OR egg OR eggs OR milk OR shellfish OR wheat)) OR "Food Hypersensitivity"[Mesh] OR asthma OR "Rhinitis, Allergic"[Mesh] OR (allergic[tiab] AND Rhiniti*[tiab]) OR "Dermatitis, Atopic"[Mesh] OR ((Dermatiti*[tiab] OR eczema[tiab]) AND Atopic[tiab]) OR (Infant* AND Eczema) OR "Immunoglobulin E"[Mesh] OR "Immunoglobulin E"[tiab] OR $\lg E[t i a b]$

for nonmedline[sb]: NOT animals by: NOT (sheep[ti] OR lamb[ti] OR lambs[ti] OR calving[ti] OR calves[ti] OR mice[ti] OR mouse[ti] OR pigs[ti] OR cows[ti] OR piglets[ti] OR cow[ti] OR piglet[ti] OR monkey[ti] OR rats[ti] OR rat[ti] OR animal*[ti])

infant* OR baby OR babies OR toddler* OR newborn*[tiab] OR "Child, Preschool"[Mesh] OR preschool*[tiab] OR pre-school*[tiab] OR "early childhood"[tiab] OR early year*[tiab] OR pre-k[tiab] OR pre-primary[tiab] OR under five*[ti] OR young child*[ti] OR prekindergarten[tiab] OR pre-kindergarten[tiab] OR weanling* OR "first two years" OR "first 2 years"

\section{Embase, Elsevier (1947 to February 2017):}

Date(s) Searched: 2/2017

Search Terms:

'complementary feeding'/exp OR

(Complementary OR supplementa* OR wean* OR transition* OR introduc* OR family) NEAR/3 (feed ${ }^{*}$ OR food ${ }^{*}$ OR beverage* OR eating OR diet)

OR

(Complementary OR transition* OR introduct* OR wean*) AND (food/exp OR 'baby food'/exp OR 'cereal'/exp OR 'dairy product'/exp OR 'egg'/exp OR 'fruit'/exp OR 
'meat'/exp OR 'sea food'/exp OR 'milk'/exp OR fish/exp OR 'poultry'/exp OR 'beverage'/exp OR 'vegetable'/exp OR nut/exp OR pea/exp OR meal/exp OR 'infant feeding'/exp)

OR

(Complementary OR supplementa* OR wean* OR transition* OR introduc*) NEAR/5 ('whole grain' OR 'whole grains' OR dairy OR egg OR eggs OR meat OR poultry OR seafood OR fruit* OR milk OR fish* OR poultry OR beverage* OR vegetables* OR pea OR peas OR nut OR nuts OR cereal OR bread* OR yog* urt $^{*}$ OR cheese* OR juice* OR rice OR soup OR legume* OR snack* OR meal*)

OR 'baby food'/de OR (solid NEAR/2 food*):ab,ti

AND

(infant*:ti,ab OR infant/exp) OR (baby OR babies OR toddler* OR newborn* OR nurser*):ti,ab OR 'newborn'/exp OR 'newborn care'/exp OR preschool*:ti,ab OR preschool:ti,ab OR 'preschool child'/exp OR 'infancy'/exp OR "early childhood":ti,ab OR "early years" OR pre-k:ti,ab OR 'nursery'/exp OR 'nursery school'/exp OR prekindergarten:ti,ab OR pre-kindergarten:ti,ab OR weanling* (includes limits below) OR ([newborn]/lim OR [infant]/lim OR [child]/lim OR [preschool]/lim)

AND ([in process]/lim OR [article]/lim OR [article in press]/lim) AND ([embase]/lim NOT [medline]/lim)

Limit to humans:

AND

'allergic asthma'/exp OR 'food allergy'/exp OR 'allergic rhinitis'/exp OR 'dermatitis'/exp OR 'eczema'/exp OR 'skin allergy'/exp OR ((allerg* OR hypersensitivity*) NEAR/3 (food OR peanut* OR nut OR nuts OR egg OR milk OR shellfish OR wheat)) OR 'immunoglobulin E'/exp OR 'immunoglobulin E':ti,ab OR 'atopy'/exp OR atopy:ti,ab OR atopic:ti,ab OR IgE:ti,ab

Cochrane Central Register of Controlled Trials, John Wiley \& Sons in the Cochrane Library (searched 9 February 2017):

Date(s) Searched: 2/9/17

Search Terms:

(feed* OR food ${ }^{*}$ OR beverage ${ }^{*}$ OR diet $^{*}$ OR 'whole grain' OR 'whole grains' OR dairy OR egg OR meat OR poultry OR seafood OR fruit* OR milk OR fish* OR poultry OR vegetables* OR pea OR beans OR legume* OR nut OR cereal OR beverage* OR

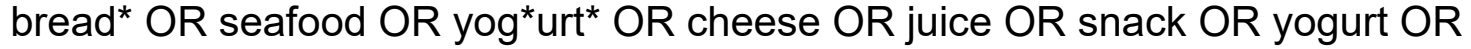
yoghurt OR nut OR nuts OR honey OR meal OR meals) NEAR/3 (Complementary OR supplementa* OR wean* OR transition* OR introduct* OR family)

OR 
[mh ^"Infant Nutritional Physiological Phenomena"] OR [mh weaning] OR ((bottle*) NOT (milk OR formula))

AND ([mh beverages] OR [mh eating] OR [mh diet] OR [mh meals] OR [mh "Food and Beverages"] OR [mh "Edible Grain"] OR [mh "Milk"] OR dairy:ti,ab OR [mh "Dairy Products"] OR [mh "Fruit"] OR [mh "Vegetables"] OR [mh "Eggs"] OR [mh bread] OR [mh honey] OR [mh "Vegetables"] OR [mh ^"Eggs"] OR [mh "egg white"] OR [mh "egg yolk"] OR [mh candy] OR [mh "Fast Foods"] OR [mh meat] OR [mh molasses] OR [mh nuts] OR [mh "Raw Foods"] OR [mh seeds])

OR

((Infant* OR baby* OR babies) NEAR/2 food*):ti,ab OR [mh "infant food"]

AND

[mh ^"Allergy and Immunology"] OR ((allerg*:ti,ab OR Hypersensitivit*:ti,ab) AND (food OR peanut OR nut OR nuts OR egg OR milk OR shellfish OR wheat)) OR [mh "Food Hypersensitivity"] OR asthma* OR [mh" Rhinitis, Allergic"] OR (allerg* NEAR/5 Rhiniti*) OR [mh "Dermatitis, Atopic"] OR ((Dermatiti* OR eczema) NEAR/5 Atopic) OR (Infant* NEAR/5 Eczema) OR [mh "Immunoglobulin E"] OR "Immunoglobulin E":ti,ab OR $\lg \mathrm{E}: \mathrm{ti}, \mathrm{ab}$

CINAHL Plus with Full Text, EBSCO (Cumulative Index to Nursing and Allied Health Literature; 1937 to 9 February 2017):

Date(s) Searched: 2/9/17

Search Terms:

209; selected 26 for downloading

(MH "Food Hypersensitivity+") OR (MH "Milk Hypersensitivity") OR (MH "Pollen-Food Allergy") OR (MH "Rhinitis, Atrophic") OR "Immunoglobulin e" OR (MH "Eczema") OR (MH "Dermatitis, Atopic")

AND

(MH "Food and Beverages+") OR (MH "Food") OR (MH "Diet") OR (MH "Eating") OR (MH "Eating Behavior") OR (MH "Taste") OR (MH "Taste Buds") OR (MH "Cereals") OR (MH "Dairy Products") OR (MH "Yogurt") OR (MH "Cheese") OR (MH "Milk") OR (MH "Eggs") OR (MH "Fruit") OR (MH "Fruit Juices") OR (MH "Meat") OR (MH "Seafood") OR (MH "Fish") OR (MH "Poultry") OR (MH "Vegetables") OR (MH "Nuts") OR (MH "Legumes") OR (MH "Bread") AND (Complementary OR supplementa* OR wean* OR transition* OR introduc*)

OR

('whole grain' OR 'whole grains' OR dairy OR egg OR eggs OR meat OR poultry OR seafood OR fruit* OR milk OR fish* OR poultry OR vegetables* OR pea OR peas OR nut OR nuts OR cereal OR beverage* OR bread* OR seafood OR yog* ${ }^{*}$ tr $^{*}$ OR

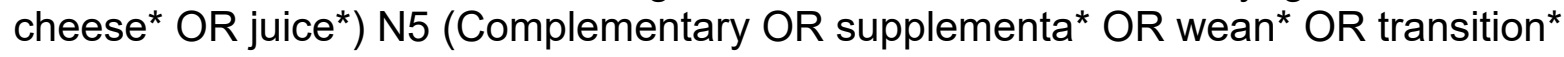


OR introduc* OR family)

OR (Infant* OR baby OR babies) N3 food*

\section{Figure 2: Flow chart of literature search and screening results}

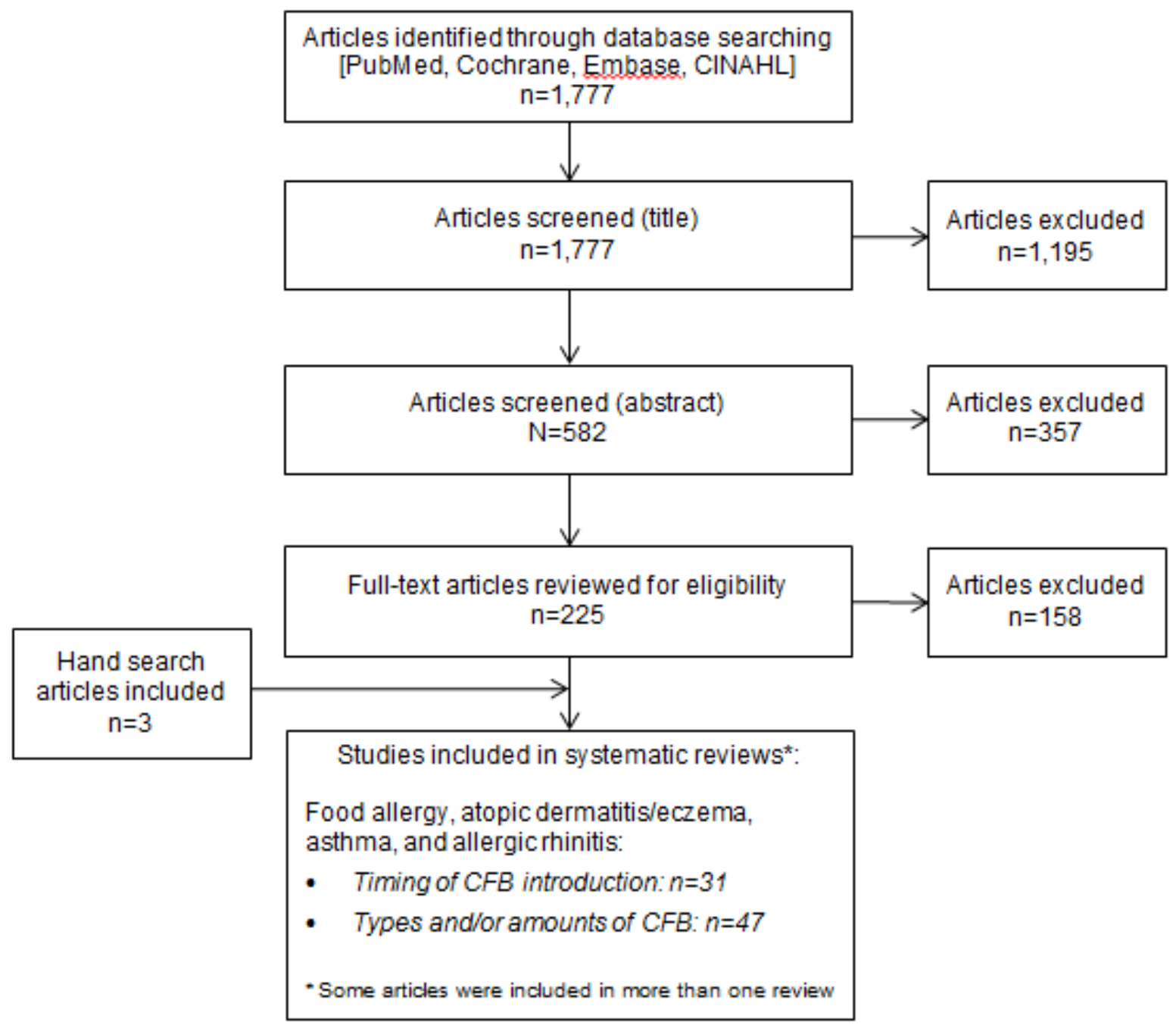

This flow chart illustrates the literature search and screening results for articles examining the relationship between complementary feeding and food allergy, atopic dermatitis/eczema, asthma, and allergic rhinitis. The results of the electronic database searches were screened independently by two NESR analysts in a step-wise manner by reviewing titles, abstracts, and full text articles to determine which articles met the criteria for inclusion. A manual search was done to ascertain articles not identified through the electronic database search. The systematic review on timing of CFB introduction included 31 articles, and the systematic review on on types and amounts of CFB consumed included 47 articles. 


\section{Excluded articles}

The table below lists the excluded articles with at least one reason for exclusion, and may not reflect all possible reasons.

\section{Table 12. Excluded articles}

\begin{tabular}{|c|c|c|}
\hline & Citation & Reasons for Exclusion \\
\hline 1 & Children's peanut allergies arising at younger ages. AORN Journal. 2008;87:624-624. & Study design \\
\hline 2 & Infants with food protein allergy tolerate soy milk earlier than cow's. Community Practitioner. 2009;82:41-41. & Study design \\
\hline 3 & Significance of food hypersensitivity in children with atopic dermatitis. Pediatr Dermatol. 1986;3:161-74. & Study design \\
\hline 4 & $\begin{array}{l}\text { Aberg, N.,Engstrom, I.,Lindberg, U.. Allergic diseases in Swedish school children. Acta Paediatr Scand. } \\
\text { 1989;78:246-52. }\end{array}$ & Study design \\
\hline 5 & $\begin{array}{l}\text { Abrams, E. M.,Becker, A. B.. Introducing solid food: age of introduction and its effect on risk of food allergy and } \\
\text { other atopic diseases. Can Fam Physician. 2013;59:721-2. }\end{array}$ & Study design \\
\hline 6 & $\begin{array}{l}\text { Alper, Z.,Sapan, N.,Ercan, I.,Canitez, Y.,Bilgel, N.. Risk factors for wheezing in primary school children in Bursa, } \\
\text { Turkey. Am J Rhinol. 2006;20:53-63. }\end{array}$ & Study design \\
\hline 7 & $\begin{array}{l}\text { Armentia, A.,Rodriguez, R.,Callejo, A.,Martin-Esteban, M.,Martin-Santos, J. M.,Salcedo, G.,Pascual, C.,Sanchez- } \\
\text { Monge, R.,Pardo, M.. Allergy after ingestion or inhalation of cereals involves similar allergens in different ages. Clin } \\
\text { Exp Allergy. 2002;32:1216-22. }\end{array}$ & $\begin{array}{l}\text { Health Status, } \\
\text { Independent Variable }\end{array}$ \\
\hline 8 & $\begin{array}{l}\text { Arshad, S. H.,Matthews, S.,Gant, C.,Hide, D. W.. Effect of allergen avoidance on development of allergic disorders } \\
\text { in infancy. Lancet. 1992;339:1493-7. }\end{array}$ & Independent Variable \\
\hline 9 & $\begin{array}{l}\text { Bardara, M.,Varin, E.,Zani, G.. Response to diet in } 130 \text { children affected with atopic dermatitis. Allergy. 1989:147- } \\
50 .\end{array}$ & Study design \\
\hline 10 & $\begin{array}{l}\text { Bardare, M.,Vaccari, A.,Allievi, E.,Brunelli, L.,Coco, F.,de Gaspari, G. C.,Flauto, U.. Influence of dietary } \\
\text { manipulation on incidence of atopic disease in infants at risk. Ann Allergy. 1993;71:366-71. }\end{array}$ & Independent Variable \\
\hline 11 & $\begin{array}{l}\text { Becker, A.,Watson, W.,Ferguson, A.,Dimich-Ward, H.,Chan-Yeung, M.. The Canadian asthma primary prevention } \\
\text { study: outcomes at } 2 \text { years of age. J Allergy Clin Immunol. 2004;113:650-6. }\end{array}$ & $\begin{array}{l}\text { Study design, Independent } \\
\text { Variable }\end{array}$ \\
\hline 12 & $\begin{array}{l}\text { Benn, C. S.,Wohlfahrt, J.,Aaby, P.,Westergaard, T.,Benfeldt, E.,Michaelsen, K. F.,Bjorksten, B.,Melbye, M.. } \\
\text { Breastfeeding and risk of atopic dermatitis, by parental history of allergy, during the first } 18 \text { months of life. Am J } \\
\text { Epidemiol. 2004;160:217-23. }\end{array}$ & Independent Variable \\
\hline 13 & $\begin{array}{l}\text { Berg, Av,Kramer, U.,Link, E.,Bollrath, C.,Heinrich, J.,Brockow, I.,Koletzko, S.,Grubl, A.,Filipiak-Pittroff, } \\
\text { B.,Wichmann, H. E.,Bauer, C. P.,Reinhardt, D.,Berdel, D.. Impact of early feeding on childhood eczema: }\end{array}$ & Independent Variable \\
\hline
\end{tabular}




\begin{tabular}{|c|c|c|}
\hline & Citation & Reasons for Exclusion \\
\hline & $\begin{array}{l}\text { development after nutritional intervention compared with the natural course - the GINIplus study up to the age of } 6 \\
\text { years. Clin Exp Allergy. 2010;40:627-36. }\end{array}$ & \\
\hline 14 & $\begin{array}{l}\text { Bergmann, R. L.,Bergmann, K. E.,Lau-Schadensdorf, S.,Luck, W.,Dannemann, A.,Bauer, C. P.,Dorsch, W.,Forster, } \\
\text { J.,Schmidt, E.,Schulz, J.,et, al. Atopic diseases in infancy. The German multicenter atopy study (MAS-90). Pediatr } \\
\text { Allergy Immunol. 1994;5:19-25. }\end{array}$ & Independent Variable \\
\hline 15 & $\begin{array}{l}\text { Bilenko, N.,Ghosh, R.,Levy, A.,Deckelbaum, R. J.,Fraser, D.. Partial breastfeeding protects Bedouin infants from } \\
\text { infection and morbidity: prospective cohort study. Asia Pac J Clin Nutr. 2008;17:243-9. }\end{array}$ & Independent Variable \\
\hline 16 & $\begin{array}{l}\text { Bion, V.,Lockett, G. A.,Soto-Ramírez, N.,Zhang, H.,Venter, C.,Karmaus, W.,Holloway, J. W.,Arshad, S. H.. } \\
\text { Evaluating the efficacy of breastfeeding guidelines on long-term outcomes for allergic disease. Allergy: European } \\
\text { Journal of Allergy and Clinical Immunology. 2016;71:661-670. }\end{array}$ & Independent Variable \\
\hline 17 & Birkbeck, J. A.. Goat milk in infant nutrition. N Z Med J. 1984;97:413-4. & Study design \\
\hline 18 & Blumberg, S.. Infant feeding: can we spice it up a bit?. J Am Diet Assoc. 2006;106:504-5. & Study design \\
\hline 19 & $\begin{array}{l}\text { Bruno, G.,Milita, O.,Ferrara, M.,Nisini, R.,Cantani, A.,Businco, L.. Prevention of atopic diseases in high risk babies } \\
\text { (long-term follow-up). Allergy Proc. 1993;14. }\end{array}$ & Independent Variable \\
\hline 20 & $\begin{array}{l}\text { Burks, K.,Jones, S.. The Canadian asthma primary prevention study: Outcomes at } 2 \text { years of age. Pediatrics. } \\
\text { 2005;116:537-4005. }\end{array}$ & Study design \\
\hline 21 & $\begin{array}{l}\text { Burr, M. L.,Limb, E. S.,Maguire, M. J.,Amarah, L.,Eldridge, B. A.,Layzell, J. C.,Merrett, T. G.. Infant feeding, } \\
\text { wheezing, and allergy: a prospective study. Arch Dis Child. 1993;68:724-8. }\end{array}$ & Independent Variable \\
\hline 22 & $\begin{array}{l}\text { Burr, M. L.,Merrett, T. G.,Dunstan, F. D.,Maguire, M. J.. The development of allergy in high-risk children. Clin Exp } \\
\text { Allergy. 1997;27:1247-53. }\end{array}$ & Independent Variable \\
\hline 23 & Calamaro, C. J.. Infant nutrition in the first year of life: tradition or science?. Pediatr Nurs. 2000;26:211-5. & Study design \\
\hline 24 & Cant, A. J.,Bailes, J. A.. How should we feed the potentially allergic infant?. Hum Nutr Appl Nutr. 1984;38:474-6. & Study design \\
\hline 25 & Challacombe, D. N.. Allergies and infant feeding. Midwife Health Visit Community Nurse. 1986;22:164-6. & Study design \\
\hline 26 & $\begin{array}{l}\text { Chandra, R. K.. Role of maternal diet and mode of infant feeding in prevention of atopic dermatitis in high risk } \\
\text { infants. Allergy. 1989:135-9. }\end{array}$ & Study design \\
\hline 27 & $\begin{array}{l}\text { Chiu, C. Y.,Liao, S. L.,Su, K. W.,Tsai, M. H.,Hua, M. C.,Lai, S. H.,Chen, L. C.,Yao, T. C.,Yeh, K. W.,Huang, J. L.. } \\
\text { Exclusive or Partial Breastfeeding for } 6 \text { Months Is Associated with Reduced Milk Sensitization and Risk of Eczema } \\
\text { in Early Childhood. Medicine (United States). 2016;95. }\end{array}$ & Independent Variable \\
\hline 28 & $\begin{array}{l}\text { Cho, H. N.,Hong, S.,Lee, S. H.,Yum, H. Y.. Nutritional status according to sensitized food allergens in children with } \\
\text { atopic dermatitis. Allergy Asthma Immunol Res. 2011;3:53-7. }\end{array}$ & Study design \\
\hline
\end{tabular}




\begin{tabular}{|c|c|c|}
\hline & Citation & Reasons for Exclusion \\
\hline 29 & $\begin{array}{l}\text { Cudowska, B.,Marcinkiewicz, S.,Kaczmarski, M.. Sensitization to cereal allergens in children with atopic dermatitis. } \\
\text { Postepy Dermatologii i Alergologii. 2011;28:181-186. }\end{array}$ & $\begin{array}{l}\text { Study design, Health } \\
\text { Status }\end{array}$ \\
\hline 30 & de Looy, A. E.. Infant nutrition. Nursing (Lond). 1986;3:446-9. & Study design \\
\hline 31 & $\begin{array}{l}\text { del-Rio Camacho, G.,Martinez Jimenez, V.,Fernandez-Cantalejo Padial, J.. Absence of clinical symptoms upon } \\
\text { introduction of egg into the diet of milk-allergic infants not previously sensitised to egg. Allergol Immunopathol } \\
\text { (Madr). 2012;40:374-8. }\end{array}$ & $\begin{array}{l}\text { Health Status, } \\
\text { Independent Variable }\end{array}$ \\
\hline 32 & $\begin{array}{l}\text { Dieguez, M. C.,Cerecedo, I.,Muriel, A.,Zamora, J.,Sanchez-Cano, M.,De la Hoz, B.. Skin prick test predictive value } \\
\text { on the outcome of a first known egg exposure in milk-allergic children. Pediatr Allergy Immunol. 2008;19:319-24. }\end{array}$ & $\begin{array}{l}\text { Health Status, } \\
\text { Independent Variable }\end{array}$ \\
\hline 33 & $\begin{array}{l}\text { Du Toit, G.,Katz, Y.,Sasieni, P.,Mesher, D.,Maleki, S. J.,Fisher, H. R.,Fox, A. T.,Turcanu, V.,Amir, T.,Zadik-Mnuhin, } \\
\text { G.,Cohen, A.,Livne, I.,Lack, G.. Early consumption of peanuts in infancy is associated with a low prevalence of } \\
\text { peanut allergy. J Allergy Clin Immunol. 2008;122:984-91. }\end{array}$ & $\begin{array}{l}\text { Study design, Dependent } \\
\text { Variable }\end{array}$ \\
\hline 34 & $\begin{array}{l}\text { Dubakiene, R.,Rudzeviciene, O.,Butiene, I.,Sezaite, I.,Petronyte, M.,Vaicekauskaite, D.,Zvirbliene, A.. Studies on } \\
\text { early allergic sensitization in the Lithuanian birth cohort. ScientificWorldJournal. 2012;2012:909524. }\end{array}$ & Independent Variable \\
\hline 35 & $\begin{array}{l}\text { Duczmal, E.,Breborowicz, A.,Duczmal, T.. The influence of specific factors on the prevalence of allergic diseases in } \\
\text { a birth cohort study [polish]. Alergia Astma Immunologia. 2011;16:96-104. }\end{array}$ & Language \\
\hline 36 & $\begin{array}{l}\text { Dunlop, A. L., Reichrtova, E.,Palcovicova, L.,Ciznar, P.,Adamcakova-Dodd, A.,Smith, S. J.,McNabb, S. J.. } \\
\text { Environmental and dietary risk factors for infantile atopic eczema among a Slovak birth cohort. Pediatr Allergy } \\
\text { Immunol. 2006;17:103-11. }\end{array}$ & Study design \\
\hline 37 & Early peanut consumption may prevent allergy. Nurse Prescribing. 2008;6:509-509. & Study design \\
\hline 38 & $\begin{array}{l}\text { Exl, B. M.,Deland, U.,Secretin, M. C.,Preysch, U.,Wall, M.,Shmerling, D. H.. Improved general health status in an } \\
\text { unselected infant population following an allergen-reduced dietary intervention programme: the ZUFF-STUDY- } \\
\text { PROGRAMME. Part II: infant growth and health status to age } 6 \text { months. ZUg-FrauenFeld. Eur J Nutr. 2000;39:145- } \\
\text { 56. }\end{array}$ & Independent Variable \\
\hline 39 & $\begin{array}{l}\text { Fadeeva, T.,Asin, J. L.,Horrillo, M. L.,Baraut, T. G.,Vela, R. F.,Conde, S. L.,Hontoria, O. E.,Valero, C. B.,Molina, A. } \\
\text { M.. Results of the oral egg-challenge test performed on two different groups of children. One group with a history, } \\
\text { suggestive of allergic reaction with egg intake and the other group sensitised to hen's egg without previous egg } \\
\text { intake. Allergol Immunopathol (Madr). 2010;38:233-40. }\end{array}$ & Independent Variable \\
\hline 40 & $\begin{array}{l}\text { Filipiak, B.,Zutavern, A.,Koletzko, S.,Von Berg, A.,Brockow, I.,Grübl, A.,Berdel, D., Reinhardt, D.,Bauer, C. } \\
\text { P.,Wichmann, H. E.,Heinrich, J.. Early solid food introduction and development of eczema in the first } 4 \text { years. } \\
\text { Results from the GINI birth cohort. Allergo Journal. 2008;17:82-83. }\end{array}$ & Language \\
\hline 41 & $\begin{array}{l}\text { Fiocchi, A.,Verga, M. C.. Early allergenic-food introduction does not reduce subsequent food allergy development. } \\
\text { J Pediatr. 2016;178:305-306. }\end{array}$ & Study design \\
\hline
\end{tabular}




\begin{tabular}{|c|c|c|}
\hline & Citation & Reasons for Exclusion \\
\hline 42 & $\begin{array}{l}\text { Flohr, C.,Nagel, G.,Weinmayr, G.,Kleiner, A.,Strachan, D. P.,Williams, H. C.. Lack of evidence for a protective effect } \\
\text { of prolonged breastfeeding on childhood eczema: lessons from the International Study of Asthma and Allergies in } \\
\text { Childhood (ISAAC) Phase Two. Br J Dermatol. 2011;165:1280-9. }\end{array}$ & Study design \\
\hline 43 & $\begin{array}{l}\text { Frank, L.,Marian, A.,Visser, M.,Weinberg, E.,Potter, P. C.. Exposure to peanuts in utero and in infancy and the } \\
\text { development of sensitization to peanut allergens in young children. Pediatr Allergy Immunol. 1999;10:27-32. }\end{array}$ & Country \\
\hline 44 & $\begin{array}{l}\text { Gabet, S.,Just, J.,Couderc, R.,Seta, N.,Momas, I.. Allergic sensitisation in early childhood: Patterns and related } \\
\text { factors in PARIS birth cohort. Int J Hyg Environ Health. 2016;219:792-800. }\end{array}$ & Dependent Variable \\
\hline 45 & $\begin{array}{l}\text { Geller-Bernstein, G.,Kenett, R.,Weisglass, L.,Tsur, S.,Lahav, M.,Levin, S.. Atopic babies with wheezy bronchitis. } \\
\text { Follow-up study relating prognosis to sequential lgE values, type of early infant feeding, exposure to parental } \\
\text { smoking and incidence of lower respiratory tract infections. Allergy. 1987;42:85-91. }\end{array}$ & $\begin{array}{l}\text { Health Status, } \\
\text { Independent Variable }\end{array}$ \\
\hline 46 & $\begin{array}{l}\text { Ghaderi, R.,Makhmalbaf, Z.. Effect of breast-feeding on the development of atopic dermatitis. Iranian Journal of } \\
\text { Allergy, Asthma and Immunology. 2005;4:129-132. }\end{array}$ & Independent Variable \\
\hline 47 & $\begin{array}{l}\text { Ghadi, A.,Dutau, G.,Rancé, F.. A sensitization study of atopic children in Marrakech. A prospective study of } 160 \\
\text { children between } 2002 \text { and } 2005 \text { [french]. Revue Francaise d'Allergologie et d'Immunologie Clinique. 2007;47:409- } \\
415 .\end{array}$ & Language \\
\hline 48 & $\begin{array}{l}\text { Gray, C. L.,Levin, M. E.,du Toit, G.. Patterns of introduction of solids in South African children with atopic dermatitis: } \\
\text { Do they affect allergy rates?. Current Allergy and Clinical Immunology. 2014;27:334-336. }\end{array}$ & $\begin{array}{l}\text { Study design, Health } \\
\text { Status }\end{array}$ \\
\hline 49 & $\begin{array}{l}\text { Greenhawt, M.,Venter, C.. Having your cake and EATing it too: early timing of multiple allergen introduction does } \\
\text { not increase the risk of developing food allergy in standard risk, breastfed infants. Evid Based Med. } 2017 .\end{array}$ & Study design \\
\hline 50 & $\begin{array}{l}\text { Greenhawt, M.. Early Allergen Introduction for Preventing Development of Food Allergy [editorial]. Jama. } \\
\text { 2016;316:1157-1159. }\end{array}$ & Study design \\
\hline 51 & $\begin{array}{l}\text { Greenhawt, M.. Early Allergen Introduction for Preventing Development of Food Allergy. Jama. 2016;316:1157- } \\
1159 .\end{array}$ & Study design \\
\hline 52 & $\begin{array}{l}\text { Guilbert, T. W.,Stern, D. A.,Morgan, W. J.,Martinez, F. D.,Wright, A. L.. Effect of breastfeeding on lung function in } \\
\text { childhood and modulation by maternal asthma and atopy. Am J Respir Crit Care Med. 2007;176:843-8. }\end{array}$ & Independent Variable \\
\hline 53 & $\begin{array}{l}\text { Gupta, R. S.,Walkner, M. M.,Greenhawt, M.,Lau, C. H.,Caruso, D.,Wang, X.,Pongracic, J. A.,Smith, B.. Food } \\
\text { Allergy Sensitization and Presentation in Siblings of Food Allergic Children. Journal of Allergy and Clinical } \\
\text { Immunology: In Practice. 2016;4:956-962. }\end{array}$ & $\begin{array}{l}\text { Health Status, } \\
\text { Independent Variable }\end{array}$ \\
\hline 54 & $\begin{array}{l}\text { Halken, S.,Host, A.,Hansen, L. G., Osterballe, O.. Effect of an allergy prevention programme on incidence of atopic } \\
\text { symptoms in infancy. A prospective study of } 159 \text { "high-risk" infants. Allergy. 1992;47:545-53. }\end{array}$ & Independent Variable \\
\hline 55 & Halken, S.. What causes allergy and asthma? The role of dietary factors. Pediatr Pulmonol Suppl. 2004;26:223-4. & Study design \\
\hline
\end{tabular}




\begin{tabular}{|c|c|c|}
\hline & Citation & Reasons for Exclusion \\
\hline 56 & $\begin{array}{l}\text { Hartman, H.,Dodd, C., Rao, M.,DeBlasio, D.,Labowsky, C.,D'Souza, S.,Lenkauskas, S.,Roeser, E.,Heffernan, } \\
\text { A.,Assa'ad, A.. Parental timing of allergenic food introduction in urban and suburban populations. Ann Allergy } \\
\text { Asthma Immunol. 2016;117. }\end{array}$ & Dependent Variable \\
\hline 57 & Hill, D. J.,Hosking, C. S.. Preventing childhood allergy. Med J Aust. 1993;158:367-9. & Study design \\
\hline 58 & Holmes, S.. Planning for the best start in life. A guide to infant feeding. Prof Nurse. 1991;6:200-5. & Study design \\
\hline 59 & $\begin{array}{l}\text { Hon, K. L. E.,Tsang, Y. C.,Poon, T. C. W.,Pong, N. H. H.,Luk, N. M.,Leung, T. N. H.,Chow, C. M.,Leung, T. F.. } \\
\text { Dairy and nondairy beverage consumption for childhood atopic eczema: What health advice to give?. Clinical and } \\
\text { Experimental Dermatology. 2016;41:129-137. }\end{array}$ & Study design, Age \\
\hline 60 & $\begin{array}{l}\text { Horwitz, A. A.,Hossain, J.,Yousef, E.. Correlates of outcome for atopic dermatitis. Ann Allergy Asthma Immunol. } \\
\text { 2009;103:146-51. }\end{array}$ & $\begin{array}{l}\text { Study design, Health } \\
\text { Status }\end{array}$ \\
\hline 61 & $\begin{array}{l}\text { Howie, P. W.,Forsyth, J. S.,Ogston, S. A.,Clark, A.,Florey, C. D.. Protective effect of breast feeding against } \\
\text { infection. Bmj. 1990;300:11-6. }\end{array}$ & Independent Variable \\
\hline 62 & $\begin{array}{l}\text { Hua, M. C.,Chen, C. C.,Liao, S. L.,Yao, T. C.,Tsai, M. H.,Lai, S. H.,Chiu, C. Y.,Yeh, K. W.,Huang, J. L.. Faecal } \\
\text { eosinophil cationic protein and serum immunoglobulin E in relation to infant feeding practices. Ann Clin Biochem. } \\
2016 .\end{array}$ & Dependent Variable \\
\hline 63 & $\begin{array}{l}\text { Ito, J.,Fujiwara, T.. Breastfeeding and risk of atopic dermatitis up to the age } 42 \text { months: a birth cohort study in } \\
\text { Japan. Ann Epidemiol. } 2014 ; 24: 267-72 \text {. }\end{array}$ & Independent Variable \\
\hline 64 & Juto, P.,Bjorksten, B.. Serum IgE in infants and influence of type of feeding. Clin Allergy. 1980;10:593-600. & Independent Variable \\
\hline 65 & $\begin{array}{l}\text { Juto, P.,Moller, C.,Engberg, S.,Bjorksten, B.. Influence of type of feeding on lymphocyte function and development } \\
\text { of infantile allergy. Clin Allergy. 1982;12:409-16. }\end{array}$ & Independent Variable \\
\hline 66 & $\begin{array}{l}\text { Kajosaari, M.. Atopy prophylaxis in high-risk infants. Prospective 5-year follow-up study of children with six months } \\
\text { exclusive breastfeeding and solid food elimination (not peer review). Adv Exp Med Biol. 1991;310:453-8. }\end{array}$ & Study design \\
\hline 67 & Kaufman, H. S.,Frick, O. L.. Prevention of asthma. Clin Allergy. 1981;11:549-53. & Independent Variable \\
\hline 68 & $\begin{array}{l}\text { Khoo, P.,Boyce, S.. Does early introduction of allergenic foods decrease the risk of food allergies?. J Paediatr } \\
\text { Child Health. 2016;52:850. }\end{array}$ & Study design \\
\hline 69 & $\begin{array}{l}\text { Kiefte-de Jong, J. C., de Vries, J. H.,Franco, O. H.,Jaddoe, V. W.,Hofman, A., Raat, H.,de Jongste, J. C.,Moll, H. A.. } \\
\text { Fish consumption in infancy and asthma-like symptoms at preschool age. Pediatrics. 2012;130:1060-8. }\end{array}$ & Dependent Variable \\
\hline 70 & $\begin{array}{l}\text { Kiefte-de Jong, J. C.,Escher, J. C.,Arends, L. R., Jaddoe, V. W.,Hofman, A., Raat, H.,Moll, H. A.. Infant nutritional } \\
\text { factors and functional constipation in childhood: the Generation R study. Am J Gastroenterol. 2010;105:940-5. }\end{array}$ & Dependent Variable \\
\hline
\end{tabular}




\begin{tabular}{|c|c|c|}
\hline & Citation & Reasons for Exclusion \\
\hline 71 & $\begin{array}{l}\text { Kim, J.,Chung, Y.,Han, Y.,Ahn, K.,Lee, S. I.. The natural history and prognostic factors of egg allergy in Korean } \\
\text { infants with atopic dermatitis. Asian Pac J Allergy Immunol. 2009;27:107-14. }\end{array}$ & Health Status \\
\hline 72 & $\begin{array}{l}\text { Kmietowicz, Zosia. Risk of peanut allergy can be reduced by } 80 \% \text { by including peanuts in infant diets. BMJ: British } \\
\text { Medical Journal. } 2015 ; 350 \text {. }\end{array}$ & Study design \\
\hline 73 & Koletzko, B.. Complementary foods and the development of food allergy. Pediatrics. 2000;106:1285. & Study design \\
\hline 74 & Koletzko, S.. 2.5 Allergy Prevention through Early Nutrition. World Rev Nutr Diet. 2015;113:113-7. & Study design \\
\hline 75 & $\begin{array}{l}\text { Koplin, J. J.,Osborne, N. J.,Wake, M.,Martin, P. E.,Gurrin, L. C.,Robinson, M. N.,Tey, D.,Slaa, M.,Thiele, L.,Miles, } \\
\text { L.,Anderson, D.,Tan, T.,Dang, T. D.,Hill, D. J.,Lowe, A. J.,Matheson, M. C.,Ponsonby, A. L.,Tang, M. L.,Dharmage, } \\
\text { S. C.,Allen, K. J.. Can early introduction of egg prevent egg allergy in infants? A population-based study. J Allergy } \\
\text { Clin Immunol. 2010;126:807-13. }\end{array}$ & $\begin{array}{l}\text { Study design, Independent } \\
\text { Variable }\end{array}$ \\
\hline 76 & $\begin{array}{l}\text { Koplin, J.,Dharmage, S. C.,Gurrin, L.,Osborne, N.,Tang, M. L.,Lowe, A. J.,Hosking, C.,Hill, D.,Allen, K. J.. Soy } \\
\text { consumption is not a risk factor for peanut sensitization. J Allergy Clin Immunol. 2008;121:1455-9. }\end{array}$ & $\begin{array}{l}\text { Study design, Independent } \\
\text { Variable }\end{array}$ \\
\hline 77 & $\begin{array}{l}\text { Kramer, B.,Raczynska, J.,Kaczmarek, J.,Lukamowicz, J.,Pasowska, R.,Puchala, B.. Genetic and environmental } \\
\text { conditions involved in assessment of the immunological state in children with atopic dermatitis. Rocz Akad Med } \\
\text { Bialymst. 1995;40:439-47. }\end{array}$ & $\begin{array}{l}\text { Study design, Independent } \\
\text { Variable }\end{array}$ \\
\hline 78 & $\begin{array}{l}\text { Kramer, M. S.,Chalmers, B.,Hodnett, E. D.,Sevkovskaya, Z.,Dzikovich, I.,Shapiro, S.,Collet, J. P.,Vanilovich, } \\
\text { I.,Mezen, I.,Ducruet, T.,Shishko, G.,Zubovich, V.,Mknuik, D.,Gluchanina, E.,Dombrovskiy, V.,Ustinovitch, A.,Kot, } \\
\text { T.,Bogdanovich, N., Ovchinikova, L.,Helsing, E.., Promotion of Breastfeeding Intervention Trial (PROBIT): a } \\
\text { randomized trial in the Republic of Belarus. Jama. 2001;285:413-20. }\end{array}$ & Independent Variable \\
\hline 79 & $\begin{array}{l}\text { Kramer, M. S.,Moroz, B.. Do breast-feeding and delayed introduction of solid foods protect against subsequent } \\
\text { atopic eczema?. J Pediatr. 1981;98:546-50. }\end{array}$ & Independent Variable \\
\hline 80 & $\begin{array}{l}\text { Krogulska, A.,Wąsowska-Królikowska, K.,Dynowski, J.. Usefulness of atopy patch tests with food allergens in } \\
\text { diagnosis of food allergy in children with dermatitis atopica. Przeglad Pediatryczny. 2007;37:245-249. }\end{array}$ & $\begin{array}{l}\text { Health Status, } \\
\text { Independent Variable, } \\
\text { Language }\end{array}$ \\
\hline 81 & $\begin{array}{l}\text { Kucukosmanoglu, E.,Yazi, D.,Yesil, O.,Akkoc, T.,Gezer, M.,Bakirci, N.,Bahceciler, N. N.,Barlan, I. B.. Prevalence of } \\
\text { egg sensitization in Turkish infants based on skin prick test. Allergol Immunopathol (Madr). 2008;36:141-4. }\end{array}$ & Study design \\
\hline 82 & $\begin{array}{l}\text { Kummeling, I.,Thijs, C.,Huber, M.,van de Vijver, L. P.,Snijders, B. E.,Penders, J.,Stelma, F.,van Ree, R.,van den } \\
\text { Brandt, P. A.,Dagnelie, P. C.. Consumption of organic foods and risk of atopic disease during the first } 2 \text { years of life } \\
\text { in the Netherlands. Br J Nutr. 2008;99:598-605. }\end{array}$ & Study design \\
\hline 83 & $\begin{array}{l}\text { Laubereau, B.,Brockow, I.,Zirngibl, A.,Koletzko, S.,Gruebl, A.,von Berg, A.,Filipiak-Pittroff, B.,Berdel, D.,Bauer, C. } \\
\text { P.,Reinhardt, D.,Heinrich, J.,Wichmann, H. E.. Effect of breast-feeding on the development of atopic dermatitis } \\
\text { during the first } 3 \text { years of life---results from the GINI-birth cohort study. J Pediatr. 2004;144:602-7. }\end{array}$ & Independent Variable \\
\hline
\end{tabular}




\begin{tabular}{|c|c|c|}
\hline & Citation & Reasons for Exclusion \\
\hline 84 & $\begin{array}{l}\text { Lee, J. M.,Neher, J. O.,Kelsberg, G.,Safranek, S.. Atopic Eczema and Early Introduction of Solid Foods. Am Fam } \\
\text { Physician. 2015;92:523-4. }\end{array}$ & Study design \\
\hline 85 & $\begin{array}{l}\text { Levin, M.,Goga, A.,Doherty, T.,Coovadia, H.,Sanders, D.,Green, R. J.,Kling, S.. Allergy and infant feeding } \\
\text { guidelines in the context of resource-constrained settings. J Allergy Clin Immunol. } 2016 .\end{array}$ & Study design \\
\hline 86 & $\begin{array}{l}\text { Luoma, R.. Environmental allergens and morbidity in atopic and non-atopic families. Acta Paediatr Scand. } \\
\text { 1984;73:448-53. }\end{array}$ & Independent Variable \\
\hline 87 & $\begin{array}{l}\text { Majeed, R., Rajar, U. D.,Shaikh, N.,Majeed, F.,Arain, A. A.. Risk factors associated with childhood asthma. J Coll } \\
\text { Physicians Surg Pak. 2008;18:299-302. }\end{array}$ & Independent Variable \\
\hline 88 & $\begin{array}{l}\text { Mauro-Martín, I. S.,Bodega-Villanueva, P.,Romero-Caamaño, E.,Micó-Moreno, V.,Garicano-Vilar, E.. Association } \\
\text { between timing of food introduction in on first year old and the prevalence of allergies [spanish]. Revista Espanola } \\
\text { de Nutricion Humana y Dietetica. 2014;18:145-154. }\end{array}$ & Language \\
\hline 89 & $\begin{array}{l}\text { Mavale-Manuel, S.,Alexandre, F.,Duarte, N.,Albuquerque, O.,Scheinmann, P.,Poisson-Salomon, A. S., de Blic, J.. } \\
\text { Risk factors for asthma among children in Maputo (Mozambique). Allergy. 2004;59:388-93. }\end{array}$ & Independent Variable \\
\hline 90 & $\begin{array}{l}\text { McKean, M.,Caughey, A. B.,Leong, R. E.,Wong, A.,Cabana, M. D.. The Timing of Infant Food Introduction in } \\
\text { Families With a History of Atopy. Clin Pediatr (Phila). 2015;54:745-51. }\end{array}$ & Dependent Variable \\
\hline 91 & $\begin{array}{l}\text { Metcalfe, J. R.,D'Vaz, N.,Makrides, M.,Gold, M. S.,Quinn, P.,West, C. E.,Loh, R.,Prescott, S. L.,Palmer, D. J.. } \\
\text { Elevated IL-5 and IL-13 responses to egg proteins predate the introduction of egg in solid foods in infants with } \\
\text { eczema. Clin Exp Allergy. 2016;46:308-16. }\end{array}$ & $\begin{array}{l}\text { Health Status, Dependent } \\
\text { Variable }\end{array}$ \\
\hline 92 & Midwinter, R. E.,Morris, A. F.,Colley, J. R.. Infant feeding and atopy. Arch Dis Child. 1987;62:965-7. & $\begin{array}{l}\text { Study design, Independent } \\
\text { Variable }\end{array}$ \\
\hline 93 & $\begin{array}{l}\text { Mihrshahi, S.,Webb, K.,Almqvist, C.,Kemp, A. S.. Adherence to allergy prevention recommendations in children } \\
\text { with a family history of asthma. Pediatr Allergy Immunol. 2008;19:355-62. }\end{array}$ & Dependent Variable \\
\hline 94 & $\begin{array}{l}\text { Milner, J. D.,Stein, D. M.,McCarter, R.,Moon, R. Y... Early infant multivitamin supplementation is associated with } \\
\text { increased risk for food allergy and asthma. Pediatrics. 2004;114:27-32. }\end{array}$ & Independent Variable \\
\hline 95 & $\begin{array}{l}\text { Miyake, Y.,Yura, A.,lki, M.. Breastfeeding and the prevalence of symptoms of allergic disorders in Japanese } \\
\text { adolescents. Clin Exp Allergy. 2003;33:312-6. }\end{array}$ & Study design \\
\hline 96 & $\begin{array}{l}\text { Moore, W. J.,Midwinter, R. E.,Morris, A. F.,Colley, J. R.,Soothill, J. F.. Infant feeding and subsequent risk of atopic } \\
\text { eczema. Arch Dis Child. 1985;60:722-6. }\end{array}$ & Independent Variable \\
\hline 97 & $\begin{array}{l}\text { Morgan, J. B.,Lucas, A.,Fewtrell, M. S.. Does weaning influence growth and health up to } 18 \text { months?. Archives of } \\
\text { Disease in Childhood: Education and Practice Edition. 2004;89:728-733. }\end{array}$ & Study design \\
\hline
\end{tabular}




\begin{tabular}{|c|c|c|}
\hline & Citation & Reasons for Exclusion \\
\hline 98 & Morin, K. H.. Food Allergies: New Evidence for Peanut Introduction. MCN Am J Matern Child Nurs. 2016;41:188. & Study design \\
\hline 99 & $\begin{array}{l}\text { Nakamura, Y.,Oki, I.,Tanihara, S.,Ojima, T.,Ito, Y.,Yamazaki, O.,Iwama, M.,Tabata, Y.,Katsuyama, K.,Sasai, } \\
\text { Y.,Nakagawa, M.,Matsushita, A.,Hossaka, K.,Sato, J.,Hidaka, Y.,Uda, H.,Nakamata, K.,Yanagawa, H.. Relationship } \\
\text { between breast milk feeding and atopic dermatitis in children. J Epidemiol. 2000;10:74-8. }\end{array}$ & $\begin{array}{l}\text { Study design, Independent } \\
\text { Variable }\end{array}$ \\
\hline 100 & & Language \\
\hline 101 & $\begin{array}{l}\text { Natsume, O.,Kabashima, S.,Nakasato, J.,Yamamoto-Hanada, K.,Narita, M.,Kondo, M.. Early introduction of egg for } \\
\text { infants with atopic dermatitis to prevent egg allergy: A double-blind placebo-controlled randomized clinical } \\
\text { trial[abstract only]. Journal of Allergy and Clinical Immunology. 2016;137. }\end{array}$ & Study design \\
\hline 102 & Neild, V.. Diet and atopic eczema. Mod Midwife. 1994;4:22. & Study design \\
\hline 103 & $\begin{array}{l}\text { Nwaru, B. I.,Erkkola, M.,Ahonen, S.,Kaila, M.,Haapala, A. M.,Kronberg-Kippila, C.,Salmelin, R.,Veijola, R., Ilonen, } \\
\text { J.,Simell, O.,Knip, M.,Virtanen, S. M.. Age at the introduction of solid foods during the first year and allergic } \\
\text { sensitization at age } 5 \text { years. Pediatrics. 2010;125:50-9. }\end{array}$ & Dependent Variable \\
\hline 104 & $\begin{array}{l}\text { Nwaru, B. I.,Takkinen, H. M.,Niemela, O.,Kaila, M.,Erkkola, M.,Ahonen, S.,Tuomi, H.,Haapala, A. M.,Kenward, M. } \\
\text { G.,Pekkanen, J.,Lahesmaa, R.,Kere, J.,Simell, O.,Veijola, R.,llonen, J.,Hyoty, H.,Knip, M.,Virtanen, S. M.. } \\
\text { Introduction of complementary foods in infancy and atopic sensitization at the age of } 5 \text { years: timing and food } \\
\text { diversity in a Finnish birth cohort. Allergy. } 2013 ; 68: 507-16 .\end{array}$ & Dependent Variable \\
\hline 105 & $\begin{array}{l}\text { Oddy, W. H.,Sherriff, J. L.. Breastfeeding, body mass index, asthma and atopy in children. Asia Pac J Public } \\
\text { Health. 2003. }\end{array}$ & Independent Variable \\
\hline 106 & $\begin{array}{l}\text { Oddy, W. H.. Breastfeeding and asthma in children: findings from a West Australian study. Breastfeed Rev. } \\
2000 ; 8: 5-11 .\end{array}$ & Independent Variable \\
\hline 107 & Oehling, A.. Importance of food allergy in childhood asthma. Allergol Immunopathol (Madr). 1981:71-3. & Study design \\
\hline 108 & $\begin{array}{l}\text { Ogbuanu, I. U.,Karmaus, W.,Arshad, S. H.,Kurukulaaratchy, R. J.,Ewart, S.. Effect of breastfeeding duration on } \\
\text { lung function at age } 10 \text { years: a prospective birth cohort study. Thorax. 2009;64:62-6. }\end{array}$ & Independent Variable \\
\hline 109 & $\begin{array}{l}\text { Ozmert, E. N.,Kale-Cekinmez, E.,Yurdakok, K.,Sekerel, B. E.. Determinants of allergic signs and symptoms in 24- } \\
\text { 48-month-old Turkish children. Turk J Pediatr. 2009;51:103-9. }\end{array}$ & Study design \\
\hline 110 & $\begin{array}{l}\text { Parihar, H.,Kumar, L.,Puri, R., Kumar, V.. The incidence of allergic diseases and feeding patterns in children upto } 2 \\
\text { years of age. Indian J Pediatr. 1984;51:7-12. }\end{array}$ & Study design \\
\hline 111 & $\begin{array}{l}\text { Paton, J.,Kljakovic, M.,Ciszek, K.,Ding, P.. Infant Feeding Practices and Nut Allergy over Time in Australian School } \\
\text { Entrant Children. Int J Pediatr. 2012;2012:675724. }\end{array}$ & Study design, Age \\
\hline
\end{tabular}




\begin{tabular}{|c|c|c|}
\hline & Citation & Reasons for Exclusion \\
\hline 112 & $\begin{array}{l}\text { Pesonen, M.,Kallio, M. J.,Ranki, A.,Siimes, M. A.. Prolonged exclusive breastfeeding is associated with increased } \\
\text { atopic dermatitis: a prospective follow-up study of unselected healthy newborns from birth to age } 20 \text { years. Clin } \\
\text { Exp Allergy. 2006;36:1011-8. }\end{array}$ & Independent Variable \\
\hline 113 & $\begin{array}{l}\text { Peters, R. L.,Allen, K. J.,Dharmage, S. C.,Lodge, C. J.,Koplin, J. J.,Ponsonby, A. L.,Wake, M.,Lowe, A. J.,Tang, M. } \\
\text { L.,Matheson, M. C.,Gurrin, L. C.. Differential factors associated with challenge-proven food allergy phenotypes in a } \\
\text { population cohort of infants: a latent class analysis. Clin Exp Allergy. 2015;45:953-63. }\end{array}$ & Study design \\
\hline 114 & $\begin{array}{l}\text { Peters, T. J.,Golding, J.. The epidemiology of childhood eczema: II. Statistical analyses to identify independent } \\
\text { early predictors. Paediatr Perinat Epidemiol. 1987;1:80-94. }\end{array}$ & Independent Variable \\
\hline 115 & $\begin{array}{l}\text { Pitt, Tj,Watson, W.,Ferguson, A.,Dimich-Ward, H.,Dybuncio, A.,Kozyrskyj, Al. Delay In The Introduction Of } \\
\text { Allergenic Foods Is Not Associated With An Increased Risk For Sensitization In A High Risk Cohort [Abstract]. } \\
\text { Journal of allergy and clinical immunology. 2010;125. }\end{array}$ & Study design \\
\hline 116 & Pohl, C. A.. Timing of cereal introduction to the infant diet. Patient Care for the Nurse Practitioner. 2006;9. & Study design \\
\hline 117 & $\begin{array}{l}\text { Pohlabeln, H.. Effect modification by familial predisposition when analyzing the influence of breastfeeding and pet } \\
\text { keeping on the development of allergic diseases in children [german]. Allergologie. 2012;35:44-53. }\end{array}$ & Language \\
\hline 118 & $\begin{array}{l}\text { Poongadan, M. N.,Gupta, N.,Kumar, R.. Dietary pattern and asthma in India. Pneumonol Alergol Pol. 2016;84:160- } \\
7 .\end{array}$ & Age \\
\hline 119 & $\begin{array}{l}\text { Poysa, L.,Pulkkinen, A.,Korppi, M.,Remes, K.,Juntunen-Backman, K.. Diet in infancy and bronchial hyperreactivity } \\
\text { later in childhood. Pediatr Pulmonol. 1992;13:215-21. }\end{array}$ & Independent Variable \\
\hline 120 & $\begin{array}{l}\text { Poysa, L.. Atopy in children with and without a family history of atopy. II. Skin reactivity. Acta Paediatr Scand. } \\
\text { 1989;78:902-6. }\end{array}$ & Independent Variable \\
\hline 121 & $\begin{array}{l}\text { Prasad, S.,Rana, R. K.,Sheth, R.,Mauskar, A. V.. A Hospital Based Study to Establish the Correlation between } \\
\text { Recurrent Wheeze and Vitamin D Deficiency Among Children of Age Group Less than } 3 \text { Years in Indian Scenario. } \\
\text { J Clin Diagn Res. 2016;10. }\end{array}$ & $\begin{array}{l}\text { Independent Variable, } \\
\text { Dependent Variable }\end{array}$ \\
\hline 122 & Pratt, H. F.. Breastfeeding and eczema. Early Hum Dev. 1984;9:283-90. & Independent Variable \\
\hline 123 & Pugh, R. J.. Infant feeding in perspective. Practitioner. 1982;226:1917-25. & Study design \\
\hline 124 & $\begin{array}{l}\text { Quah, P. L.,Loo, E. X.,Lee, G. N.,Kuo, I. C.,Gerez, I.,Llanora, G. V.,Chan, Y. H.,Aw, M.,Shek, L. P.,Lee, B. W.. } \\
\text { Clinical phenotype and allergen sensitization in the first } 2 \text { years as predictors of atopic disorders at age } 5 \text { years. } \\
\text { World Allergy Organ J. 2015;8:33. }\end{array}$ & Independent Variable \\
\hline 125 & Rosenberg, K.. Early Introduction Reduces Risk of Some Food Allergies. Am J Nurs. 2017;117:65-66. & Study design \\
\hline
\end{tabular}




\begin{tabular}{|c|c|c|}
\hline & Citation & Reasons for Exclusion \\
\hline 126 & $\begin{array}{l}\text { Roslan, K.,Szczepanski, M.,Kaczmarski, M.,Zapolska, B.,Uscinowicz, M.,Wasilewska, J.,Solarz, E.. Environmental } \\
\text { and constitutional conditions and food hypersensitivity in children. Rocz Akad Med Bialymst. 1995;40:448-51. }\end{array}$ & $\begin{array}{l}\text { Study design, Health } \\
\text { Status }\end{array}$ \\
\hline 127 & $\begin{array}{l}\text { Satwani, H.,Rehman, A.,Ashraf, S.,Hassan, A.. Is serum total lgE levels a good predictor of allergies in children?. J } \\
\text { Pak Med Assoc. 2009;59:698-702. }\end{array}$ & Study design \\
\hline 128 & $\begin{array}{l}\text { Sausenthaler, S.,Heinrich, J.,Koletzko, S.. Early diet and the risk of allergy: What can we learn from the prospective } \\
\text { birth cohort studies GINIplus and LISAplus?. American Journal of Clinical Nutrition. 2011;94. }\end{array}$ & Study design \\
\hline 129 & $\begin{array}{l}\text { Sherriff, A.,Peters, T. J.,Henderson, J.,Strachan, D.. Risk factor associations with wheezing patterns in children } \\
\text { followed longitudinally from birth to 3(1/2) years. Int J Epidemiol. 2001;30:1473-84. }\end{array}$ & Independent Variable \\
\hline 130 & $\begin{array}{l}\text { Shohet, L.,Shahar, E.,Davidson, S.. Breast feeding as prophylaxis for atopic eczema: a controlled study of } 368 \\
\text { cases. Acta Paediatr Hung. 1985;26:35-9. }\end{array}$ & Independent Variable \\
\hline 131 & $\begin{array}{l}\text { Siltanen, M.,Kajosaari, M.,Poussa, T.,Saarinen, K. M.,Savilahti, E.. A dual long-term effect of breastfeeding on } \\
\text { atopy in relation to heredity in children at } 4 \text { years of age. Allergy. 2003;58:524-30. }\end{array}$ & Independent Variable \\
\hline 132 & $\begin{array}{l}\text { Silvers, K. M.,Frampton, C. M.,Wickens, K.,Pattemore, P. K.,Ingham, T.,Fishwick, D.,Crane, J.,Town, G. I.,Epton, } \\
\text { M. J.. Breastfeeding protects against current asthma up to } 6 \text { years of age. J Pediatr. 2012;160. }\end{array}$ & Independent Variable \\
\hline 133 & Smith, P.. Dietary prevention of food allergies in infants. Australian Journal of Pharmacy. 2012;93:80-83. & Study design \\
\hline 134 & $\begin{array}{l}\text { Sybilski, A. J.,Doboszyńska, A.,Samoliński, B.. Influence of selected risk factors on the development of allergy } \\
\text { during the first year of life [polish]. Przeglad Pediatryczny. 2008;38:13-19. }\end{array}$ & $\begin{array}{l}\text { Independent Variable, } \\
\text { Language }\end{array}$ \\
\hline 135 & Ta, V.,Laubach, S.. Introduction of complementary foods and the relationship to food allergy. Pediatrics. 2014. & Study design \\
\hline 136 & Taitz, L.. Feeding children in the first year of life. Community Nurse. 1990;26:81-84. & Study design \\
\hline 137 & $\begin{array}{l}\text { Takemura, Y.,Sakurai, Y.,Honjo, S.,Kusakari, A.,Hara, T.,Gibo, M.,Tokimatsu, A.,Kugai, N.. Relation between } \\
\text { breastfeeding and the prevalence of asthma : the Tokorozawa Childhood Asthma and Pollinosis Study. Am J } \\
\text { Epidemiol. 2001;154:115-9. }\end{array}$ & Independent Variable \\
\hline 138 & $\begin{array}{l}\text { Tan, Jw- L.,Valerio, C.,Barnes, E. H.,Asperen, P. P.,Kakakios, A. M.,Campbell, D. E.. Early introduction of dietary } \\
\text { egg reduces egg sensitization at } 12 \text { months of age in infants at risk of allergic disease [abstract only]. Journal of } \\
\text { Allergy and Clinical Immunology. } 2016 ; 137 .\end{array}$ & Study design \\
\hline 139 & Taylor, B.. Infant feeding and allergy: fact and fiction. Midwife Health Visit Community Nurse. 1984;20:354-60. & Study design \\
\hline 140 & $\begin{array}{l}\text { Tromp,, II,Briede, S.,Kiefte-de Jong, J. C., Renders, C. M.,Jaddoe, V. W.,Franco, O. H.,Hofman, A.,Raat, H.,Moll, H. } \\
\text { A.. Factors associated with the timing of introduction of complementary feeding: the Generation R Study. Eur J Clin } \\
\text { Nutr. 2013;67:625-30. }\end{array}$ & Dependent Variable \\
\hline
\end{tabular}




\begin{tabular}{|c|c|c|}
\hline & Citation & Reasons for Exclusion \\
\hline 141 & $\begin{array}{l}\text { van Odijk, J.,Hulthen, L.,Ahlstedt, S.,Borres, M. P.. Introduction of food during the infant's first year: a study with } \\
\text { emphasis on introduction of gluten and of egg, fish and peanut in allergy-risk families. Acta Paediatr. 2004;93:464- } \\
70 .\end{array}$ & $\begin{array}{l}\text { Study design, Dependent } \\
\text { Variable }\end{array}$ \\
\hline 142 & $\begin{array}{l}\text { Ventura, A.,De Seta, L.,Martelossi, S.,Florean, P.,Maggiore, G.,Salvatore, C. M.,Berzioli, M.,Guidobaldi, } \\
\text { G.,Lorenzini, G.,Peressini, P.,Pesenti, P.,Rollo, G.,Sacher, B.,Santoro, L.,Stanzione, V.,Stranamore, D.,Zannerio, } \\
\text { E.. Soy allergy and DSCG in atopic eczema: "much ado about nothing"?. Pediatr Med Chir. 1996;18:283-8. }\end{array}$ & $\begin{array}{l}\text { Health Status, } \\
\text { Independent Variable }\end{array}$ \\
\hline 143 & $\begin{array}{l}\text { Verduci, E.,Banderali, G.,Peroni, D.,Lassandro, C.,Radaelli, G.. Duration of exclusive breastfeeding and wheezing } \\
\text { in the first year of life: A longitudinal study. Allergol Immunopathol (Madr). } 2016 .\end{array}$ & Independent Variable \\
\hline 144 & Visser, H. K.. Dietary influences on infection and allergy in infants: introduction. J Nutr. 2008;138. & Study design \\
\hline 145 & Waddell, L.. Introduction of solids in babies at risk of allergies. J Fam Health Care. 2014;24:22-7. & Study design \\
\hline 146 & $\begin{array}{l}\text { Waidyatillake, N. T.,Simpson, J. A.,Allen, K. J.,Lodge, C. J.,Dharmage, S. C.,Abramson, M. J.,De Livera, A. } \\
\text { M.,Matheson, M. C.,Erbas, B.,Hill, D. J.,Lowe, A. J.. The effect of breastfeeding on lung function at } 12 \text { and } 18 \\
\text { years: A prospective cohort study. European Respiratory Journal. 2016;48:125-132. }\end{array}$ & Independent Variable \\
\hline 147 & $\begin{array}{l}\text { Wegienka, G., Ownby, D. R.,Havstad, S.,Williams, L. K.,Johnson, C. C.. Breastfeeding history and childhood allergic } \\
\text { status in a prospective birth cohort. Ann Allergy Asthma Immunol. 2006;97:78-83. }\end{array}$ & Independent Variable \\
\hline 148 & $\begin{array}{l}\text { West, C. E.,Hammarstrom, M. L.,Hernell, O.. Probiotics during weaning reduce the incidence of eczema. Pediatr } \\
\text { Allergy Immunol. 2009;20:430-7. }\end{array}$ & Independent Variable \\
\hline 149 & $\begin{array}{l}\text { White, J. M.,McFadden, J. P.. Contact allergens in food ingredients and additives: atopy and the hapten-atopy } \\
\text { hypothesis. Contact Dermatitis. 2008;58:245-6. }\end{array}$ & $\begin{array}{l}\text { Study design, Dependent } \\
\text { Variable }\end{array}$ \\
\hline 150 & $\begin{array}{l}\text { Woicka-Kolejwa, K.,Zaczeniuk, M.,Majak, P.,Pawlowska-Iwanicka, K.,Kopka, M.,Stelmach, W.,Jerzynska, } \\
\text { J.,Stelmach, I.. Food allergy is associated with recurrent respiratory tract infections during childhood. Postepy } \\
\text { Dermatol Alergol. 2016;33:109-13. }\end{array}$ & $\begin{array}{l}\text { Study design, Dependent } \\
\text { Variable }\end{array}$ \\
\hline 151 & $\begin{array}{l}\text { Woś, H.,Cholewa, Z.,Brozek, G.. The influence of breast-feeding on the rate of occurrence of bronchial asthma in } \\
\text { children at the younger school age. Pediatria Wspolczesna. } 2003 ; 5: 21-27 \text {. }\end{array}$ & $\begin{array}{l}\text { Independent Variable, } \\
\text { Language }\end{array}$ \\
\hline 152 & $\begin{array}{l}\text { Wright, A. L.,Holberg, C. J.,Martinez, F. D.,Halonen, M.,Morgan, W.,Taussig, L. M.. Epidemiology of physician- } \\
\text { diagnosed allergic rhinitis in childhood. Pediatrics. 1994;94:895-901. }\end{array}$ & Dependent Variable \\
\hline 153 & $\begin{array}{l}\text { Wright, A. L.,Holberg, C. J.,Taussig, L. M.,Martinez, F. D.. Factors influencing the relation of infant feeding to } \\
\text { asthma and recurrent wheeze in childhood. Thorax. 2001;56:192-7. }\end{array}$ & Independent Variable \\
\hline 154 & $\begin{array}{l}\text { Wright, A. L.,Holberg, C. J.,Taussig, L. M.,Martinez, F.. Maternal asthma status alters relation of infant feeding to } \\
\text { asthma in childhood (not peer rev jl). Adv Exp Med Biol. 2000;478:131-7. }\end{array}$ & Study design \\
\hline
\end{tabular}




\begin{tabular}{|l|l|l|}
\hline & Citation & Reasons for Exclusion \\
\hline 155 & $\begin{array}{l}\text { Xu, M.,Wang, Y.,Dai, Z.,Zhang, Y.,Li, Y.,Wang, J.. Comparison of growth and nutritional status in infants receiving } \\
\text { goat milk-based formula and cow milk-based formula: A randomized, double-blind study. Food and Nutrition } \\
\text { Research. 2015;59. }\end{array}$ & $\begin{array}{l}\text { Independent Variable, } \\
\text { Dependent Variable }\end{array}$ \\
\hline 156 & $\begin{array}{l}\text { Yamakawa, M.,Yorifuji, T.,Kato, T.,Yamauchi, Y.,Doi, H.. Breast-feeding and hospitalization for asthma in early } \\
\text { childhood: a nationwide longitudinal survey in Japan. Public Health Nutr. 2015;18:1756-61. }\end{array}$ & Independent Variable \\
\hline 157 & $\begin{array}{l}\text { Young, H. B.,Buckley, A. E.,Hamza, B.,Mandarano, C.. Milk and lactation: some social and developmental } \\
\text { correlates among 1,000 infants. Pediatrics. 1982;69:169-75. }\end{array}$ & $\begin{array}{l}\text { Study design, Independent } \\
\text { Variable }\end{array}$ \\
\hline 158 & $\begin{array}{l}\text { Zeiger, R. S.,Heller, S.,Mellon, M. H.,Forsythe, A. B.,O'Connor, R. D.,Hamburger, R. N.,Schatz, M.. Effect of } \\
\text { combined maternal and infant food-allergen avoidance on development of atopy in early infancy: a randomized } \\
\text { study. J Allergy Clin Immunol. 1989;84:72-89. }\end{array}$ & Independent Variable \\
\hline
\end{tabular}

